Pacific

Journal of

Mathematics

\title{
THREE-DIMENSIONAL TRANSONIC SHOCKS IN A NOZZLE
}

Zhouping Xin AND HUicheng Yin 


\title{
THREE-DIMENSIONAL TRANSONIC SHOCKS IN A NOZZLE
}

\author{
Zhouping Xin AND HUICHENG Yin
}

\begin{abstract}
This paper concerns the following transonic shock phenomena in a threedimensional de Laval nozzle described by Courant and Friedrichs: Given the appropriately large receiver pressure $p_{r}$, if the upstream flow is still supersonic behind the throat of the nozzle, then at a certain place in the widening part of the nozzle a shock front intervenes, and the gas is compressed and slowed down to subsonic speed. The position and the strength of the shock front are automatically adjusted so that the end pressure at the exit becomes $p_{r}$. We study this problem for the inviscid steady potential equation. In this case, the transonic shock is a free boundary dividing the hyperbolic region and the elliptic region in the nozzle. One main result is that for a general class of nozzles, such a transonic shock solution is unique if the shock exists and is assumed to pass through a fixed point. We also construct a class of de Laval nozzles such that the transonic shock phenomena do not occur for the generally given large pressures at the exit for the potential flow model.
\end{abstract}

\section{Introduction and the main results}

This is a continuation of our study of the well-posedness of the problem of a multidimensional transonic shock to the steady flow through a general curved nozzle [Xin and Yin 2005a]. Our focus is on transonic flows with shocks in a general three-dimensional nozzle, which is an important subject in gas dynamics [Bers 1954; Courant and Friedrichs 1948; Fletcher 1991a; 1991b]. In particular, we are concerned with the following transonic phenomena in a De Laval nozzle as posed by Courant and Friedrichs [1948, p. 386]: Given an appropriately large receiver pressure $p_{r}$, if the upstream flow is still supersonic behind the throat of the nozzle,

MSC2000: primary 35L67, 35L65, 35L70; secondary 76N15.

Keywords: transonic flow, ill-posedness, well-posedness, potential equation, multidimensional shock wave, nozzle.

This research was supported in part by the Zheng Ge Ru Foundation when Yin Huicheng was visiting The Institute of Mathematical Sciences at The Chinese University of Hong Kong. Xin is supported in part by Hong Kong RGC Earmarked Research Grants CUHK-4040/06P, CUHK-4028/04, and RGC Central Allocation Grant CA05/06.SC01. Yin is supported in part by NNSF of China and the doctoral program of NEM of China. 
then at a certain place in the widening part of the nozzle, a shock front intervenes, and the gas is compressed and slowed down to subsonic speed. The position and the strength of the shock front are automatically adjusted so that the end pressure at the exit becomes $p_{r}$.

In [Xin and Yin 2005a], we established the well-posedness of the structure mentioned above of the transonic flow with shocks in a general two-dimensional nozzle for a class of pressures which are induced by appropriate boundary conditions at the exit of the nozzle. However, as shown by Courant and Friedrichs [1948, p. 377], the flow through a duct should be considered as a steady, isentropic, irrotational flow with cylindrical symmetry and should be determined by solving the threedimensional potential flow equations with appropriate boundary conditions. Thus, one major goal of this paper is to treat the well-posedness or ill-posedness of such a transonic flow pattern with a multidimensional shock in a general threedimensional nozzle with a slowly-varying cross section. For other discussions on transonic flows and transonic flows with shocks and recent studies on multidimensional transonic shocks, refer to [Xin and Yin 2005a; 2005b; Xin et al. 2008] and the references therein; see also [Čanić et al. 2000; Chen and Feldman 2003; Morawetz 1956; 1958; 1964; 1957; 1986].

Suppose that there is a uniform supersonic flow $\left(u_{1}, u_{2}, u_{3}\right)=\left(q_{-}, 0,0\right)$ with constant density $\rho_{0}>0$ and which comes from minus infinity; suppose also the flow enters the nozzle. We assume throughout that the nozzle wall is of a small perturbation of a cylindrical surface $\left\{x: x_{2}^{2}+x_{3}^{2}=1\right.$ for $\left.-1 \leq x_{1} \leq 1\right\}$. In addition, the flow in the nozzle is assumed to be irrotational and isentropic; see [Alt et al. 1985; Bers 1954; Courant and Friedrichs 1948; Majda 1991] and again the papers of Morawetz.

Let $\varphi(x)$ be the potential of velocity, that is, $\left(\partial_{1} \varphi, \partial_{2} \varphi, \partial_{3} \varphi\right)=\left(u_{1}, u_{2}, u_{3}\right)$. Then Bernoulli's law implies

$$
\frac{1}{2}|\nabla \varphi|^{2}+h(\rho) \equiv C_{0}=\frac{1}{2} q_{-}^{2}+h\left(\rho_{0}\right),
$$

where $h(\rho)$ is the specific enthalpy. For the given equation of state $P=P(\rho)$ with $P^{\prime}(\rho)=c^{2}(\rho)>0$ for $\rho>0$, we have $h^{\prime}(\rho)=c^{2}(\rho) / \rho$.

Since $h^{\prime}(\rho)>0$, one then can define the inverse function of $h(\rho)$ to be $H(s)$, namely,

$$
\rho=H\left(C_{0}-\frac{1}{2}|\nabla \varphi|^{2}\right) .
$$

The equation of continuity becomes

$$
\sum_{i=1}^{3} \partial_{i}\left(\partial_{i} \varphi H\right)=0,
$$


which can be rewritten as

$$
\sum_{i=1}^{3}\left(\left(\partial_{i} \varphi\right)^{2}-c^{2}\right) \partial_{i}^{2} \varphi+2 \sum_{1 \leq i<j \leq 3} \partial_{i} \varphi \partial_{j} \varphi \partial_{i j}^{2} \varphi=0 .
$$

It is easy to verify that (1-3) is strictly hyperbolic for $|\nabla \varphi|>c(\rho)$ and strictly elliptic for $|\nabla \varphi|<c(\rho)$.

Suppose that the wall of the nozzle is given by $\left(x_{2}^{2}+x_{3}^{2}\right)^{1 / 2}=f(x)$ for $-1 \leq$ $x_{1} \leq 1$, such that

$$
\left|\nabla_{x}^{\alpha}(f(x)-1)\right| \leq \varepsilon \quad \text { for }-1 \leq x_{1} \leq 1 \text { and }|\alpha| \leq k_{0},
$$

where $k_{0} \in \mathbb{N}$ and $k_{0} \geq 7$.

Without loss of generality and for convenience, we assume that

$$
\begin{aligned}
f\left(-1, x_{2}, x_{3}\right) & =1,\left.\quad \nabla_{x}^{\alpha} f(x)\right|_{x_{1}=-1}=0 \quad \text { for } 1 \leq|\alpha| \leq k_{0} . \\
f\left(1, x_{2}, x_{3}\right) & =1,
\end{aligned}
$$

When the uniform supersonic flow $\left(q_{-}, 0,0\right)$ enters the entry of the nozzle, then the potential $\varphi_{-}(x)$ in the nozzle will be determined by the initial boundary value problem for a quasilinear wave equation given by

$$
\left\{\begin{array}{l}
\sum_{i=1,2,3}\left(\left(\partial_{i} \varphi_{-}\right)^{2}-c_{-}^{2}\right) \partial_{i}^{2} \varphi_{-}+2 \sum_{1 \leq i<j \leq 3} \partial_{i} \varphi_{-} \partial_{j} \varphi_{-} \partial_{i j}^{2} \varphi_{-}=0, \\
\left.\varphi_{-}\right|_{x_{1}=-1}=-q_{-}, \\
\left.\partial_{1} \varphi_{-}\right|_{x_{1}=-1}=q_{-}, \\
\partial_{1} f \partial_{1} \varphi_{-}+\sum_{i=2,3}\left(\partial_{i} f-x_{i} / f\right) \partial_{i} \varphi_{-}=0 \quad \text { on }\left(x_{2}^{2}+x_{3}^{2}\right)^{1 / 2}=f(x),
\end{array}\right.
$$

where $c_{-}=c\left(\rho_{-}\right)$and $\rho_{-}=H\left(C_{0}-\frac{1}{2}\left|\nabla \varphi_{-}\right|^{2}\right)$.

It follows from Lemma 2.1 that (1-6) has a $C^{5}$ solution $\varphi_{-}(x)$ in the nozzle $\left\{\left(x_{1}, x_{2}, x_{3}\right):-1 \leq x_{1} \leq 1,\left(x_{2}^{2}+x_{3}^{2}\right)^{1 / 2} \leq f(x)\right\}$. Also $\left|\nabla_{x}^{\alpha}\left(\varphi_{-}(x)-q_{-} x_{1}\right)\right| \leq C \varepsilon$ holds for $|\alpha| \leq 5$.

Suppose the pressure $\widetilde{P}_{+}\left(x_{2}, x_{3}\right)=P\left(\tilde{\rho}_{+}\left(x_{2}, x_{3}\right)\right)$ at the exit $x_{1}=1$ of the nozzle is appropriately larger than that in the entry, where

$$
\tilde{\rho}_{+}\left(x_{2}, x_{3}\right) \in C^{4}\left(\left\{\left(x_{2}, x_{3}\right):\left(x_{2}^{2}+x_{3}^{2}\right)^{1 / 2} \leq f\left(1, x_{2}, x_{3}\right)\right\}\right)
$$

is a small perturbation of the constant density $\rho_{+}$or, more precisely,

$$
\left|\nabla_{x_{2}, x_{3}}^{\alpha}\left(\tilde{\rho}_{+}\left(x_{2}, x_{3}\right)-\rho_{+}\right)\right| \leq \varepsilon \quad \text { for } 0 \leq|\alpha| \leq 4,
$$

where the density $\rho_{+}$and the constant velocity $|\nabla \varphi|=q_{+}$satisfy the relations

$$
\frac{1}{2} q_{+}^{2}+h\left(\rho_{+}\right)=C_{0}, \quad \rho_{+} q_{+}=\rho_{0} q_{-}, \quad q_{+}<c\left(\rho_{+}\right) .
$$


Then we expect that there appears a transonic shock $\Sigma: x_{1}=\xi\left(x_{2}, x_{3}\right)$ in the nozzle. To assure uniqueness of the flow pattern as in [Xin and Yin 2005a], we also require that the shock $\Sigma$ goes through a specified point $\tilde{x}^{0}=\left(0, \tilde{x}_{2}^{0}, \tilde{x}_{3}^{0}\right)$ at the fixed boundary, that is,

$$
\xi\left(\tilde{x}_{2}^{0}, \tilde{x}_{3}^{0}\right)=0, \quad \text { with }\left(\left(\tilde{x}_{2}^{0}\right)^{2}+\left(\tilde{x}_{3}^{0}\right)^{2}\right)^{1 / 2}=f\left(\tilde{x}^{0}\right) .
$$

Let $\varphi_{+}(x)$ be the velocity potential across the shock $\Sigma$. Then the potential is continuous across the shock $\Sigma$ [Bers 1954; Courant and Friedrichs 1948], that is,

$$
\varphi_{+}(x)=\varphi_{-}(x) \quad \text { for } x \in \Sigma
$$

and $\nabla \varphi$ must satisfy the Rankine-Hugoniot condition

$$
\left[\partial_{1} \varphi H\right]-\sum_{i=2,3} \partial_{i} \xi\left[\partial_{i} \varphi H\right]=0 \quad \text { on } \Sigma .
$$

Furthermore, the entropy should satisfy the physical condition

$$
H\left(C_{0}-\frac{1}{2}\left|\nabla \varphi_{-}\right|^{2}\right)<H\left(C_{0}-\frac{1}{2}\left|\nabla \varphi_{+}\right|^{2}\right) \quad \text { on } \Sigma .
$$

At the exit of the nozzle, the given pressure satisfies

$$
H\left(C_{0}-\frac{1}{2}\left|\nabla \varphi_{+}\right|^{2}\right)=\tilde{\rho}_{+}\left(x_{2}, x_{3}\right) \quad \text { on } x_{1}=1 .
$$

Finally, the no-flow boundary condition on the wall of the nozzle says

$$
\partial_{1} f \partial_{1} \varphi_{+}+\sum_{i=2,3}\left(\partial_{i} f-x_{i} / f\right) \partial_{i} \varphi_{+}=0 \quad \text { on }\left(x_{2}^{2}+x_{3}^{2}\right)^{1 / 2}=f(x) .
$$

We will use the notations

$$
\begin{aligned}
\Omega & =\left\{\left(x_{1}, x_{2}, x_{3}\right):-1<x_{1}<1,\left(x_{2}^{2}+x_{3}^{2}\right)^{1 / 2}<f(x)\right\}, \\
\Omega_{+} & =\left\{\left(x_{1}, x_{2}, x_{3}\right): \xi\left(x_{2}, x_{3}\right)<x_{1}<1,\left(x_{2}^{2}+x_{3}^{2}\right)^{1 / 2}<f(x)\right\}, \\
S & =\left\{\left(x_{2}, x_{3}\right):\left(\xi\left(x_{2}, x_{3}\right), x_{2}, x_{3}\right) \in \Sigma\right\},
\end{aligned}
$$

where the last is the projection of the shock surface $\Sigma$ on the $\left(x_{2}, x_{3}\right)$-plane. Also,

$$
\begin{aligned}
\widetilde{\Gamma}_{1} & =\Sigma \cap\left\{\left(x_{1}, x_{2}, x_{3}\right):\left(x_{2}^{2}+x_{3}^{2}\right)^{1 / 2}=f(x)\right\}, \\
\widetilde{\Gamma}_{2} & =\left\{\left(1, x_{2}, x_{3}\right):\left(x_{2}^{2}+x_{3}^{2}\right)^{1 / 2}=f\left(1, x_{2}, x_{3}\right)\right\}, \\
\left|d_{\Gamma_{1}}\right| & =\operatorname{dist}\left(x, \widetilde{\Gamma}_{1}\right) \quad \text { for } x \in \Sigma \text { and }\left(x_{2}, x_{3}\right) \in S, \\
\left|d_{x}\right| & =\min \left\{\operatorname{dist}\left(x, \widetilde{\Gamma}_{1}\right), \operatorname{dist}\left(x, \widetilde{\Gamma}_{2}\right)\right\} \quad \text { for } x \in \Omega_{+} .
\end{aligned}
$$

Our first main result concerns the uniqueness of the solution to the Equation (1-3) with the boundary conditions (1-8)-(1-13). 
Theorem 1.1 (uniqueness). Suppose that (1-4), (1-5), and (1-7) hold. Then for suitably small $\varepsilon>0$, Equation (1-3) with boundary conditions (1-8)-(1-13) has no more than one pair of solutions $\left(\varphi_{+}(x), \xi\left(x_{2}, x_{3}\right)\right)$ with the following regularity properties:

(i) For $k=2,3$ and $\left(x_{2}, x_{3}\right) \in S$,

$$
\begin{aligned}
& \xi\left(x_{2}, x_{3}\right) \in C^{1,1-\delta_{0}}(\bar{S}) \cap C^{3}(S), \quad\left|\nabla_{x_{2}, x_{3}}^{k} \xi\left(x_{2}, x_{3}\right)\right| \leq \frac{C \varepsilon}{\left|d_{\widetilde{\Gamma}_{1}}\right|^{k-2+\delta_{0}}} . \\
& \left\|\xi\left(x_{2}, x_{3}\right)\right\|_{C^{1,1-\delta_{0}}(\bar{S})} \leq C \varepsilon
\end{aligned}
$$

Here and below $\delta_{0} \in(0,1 / 3)$ is a fixed constant.

(ii) $\varphi_{+}(x) \in C^{1,1-\delta_{0}}\left(\bar{\Omega}_{+}\right) \cap C^{3}\left(\Omega_{+}\right)$such that

$$
\begin{aligned}
& \left\|\varphi_{+}(x)-q_{+} x_{1}\right\|_{C^{1,1-\delta_{0}\left(\bar{\Omega}_{+}\right)}} \leq C \varepsilon, \\
& \left|\nabla_{x}^{k} \varphi_{+}(x)\right| \leq \frac{C \varepsilon}{\left|d_{x}\right|^{k-2+\delta_{0}}} \quad \text { for } k=2,3 \text { and } x \in \Omega_{+} .
\end{aligned}
$$

Remark 1.2. It follows from the regularity theory of the second order elliptic equations with the cornered boundaries (see for example [Lieberman 1988]) that the assumptions on the regularity of the solution $\left(\varphi_{+}(x), \xi\left(x_{2}, x_{3}\right)\right)$ in Theorem 1.1 are plausible. See also Theorem 1.8 below.

Remark 1.3. If the end pressure $\tilde{\rho}\left(x_{2}, x_{3}\right)$ in (1-12) is given on a $C^{4}$-smooth surface $x_{1}=g\left(x_{2}, x_{3}\right)$ with $\left|\nabla_{x_{2}, x_{3}}^{\alpha}\left(g\left(x_{2}, x_{3}\right)-1\right)\right| \leq \varepsilon$ for $0 \leq|\alpha| \leq 4$ and $\left(x_{2}, x_{3}\right) \in$ $\left\{\left(x_{2}, x_{3}\right):\left(x_{2}^{2}+x_{3}^{2}\right)^{1 / 2} \leq f\left(1, x_{2}, x_{3}\right)\right\}$, then by an analogous proof, one can show that Theorem 1.1 also holds in this case. This remark will be useful in proving Theorem 1.5 below.

Remark 1.4. To prove Theorem 1.1 (see Section 2), we will reformulate the problem (1-3) with (1-8)-(1-13) by introducing the partial hodograph transformation

$$
\begin{aligned}
& X_{1}=1-\frac{1-x_{1}}{1-x_{1}+\varphi_{-}(x)-\varphi_{+}(x)}, \\
& X_{i}=x_{i} / f(x) \quad \text { for } i=2,3 .
\end{aligned}
$$

The transformation (1-14) changes the domain $\Omega_{+}$into

$$
Q_{+}=\left\{\left(X_{1}, X_{2}, X_{3}\right): 0<X_{1}<1, X_{2}^{2}+X_{3}^{2}<1\right\}
$$


Define a new unknown function $V(X)=1-x_{1}+\varphi_{-}(x)-\varphi_{+}(x)$. Then it follows from a direct computation that Equation (1-3) with the boundary conditions (1-8)(1-10) and (1-12)-(1-13) can be reformulated as (see Section 2)

$$
\begin{aligned}
\sum_{i, j=1,2,3} a_{i j}\left(X, V, \nabla_{X} V\right) \partial_{X_{i} X_{j}}^{2} V+F_{0}\left(X, V, \nabla_{X} V\right) & =0 & & \text { in } Q_{+}, \\
G\left(X, V, \nabla_{X} V\right) & =0 & & \text { on } X_{1}=0, \\
H\left(C_{0}-\frac{1}{2}\left(\left(1+\partial_{x_{1}} V-\partial_{1} \varphi_{-}\right)^{2}+\sum_{i=2,3}\left(\partial_{x_{i}} V-\partial_{i} \varphi_{-}\right)^{2}\right)\right) & =\tilde{\rho}_{+}(x) & & \text { on } X_{1}=1,
\end{aligned}
$$

and, on $X_{2}^{2}+X_{3}^{2}=1$,

$$
\begin{aligned}
\sum_{j=1,2,3}\left(\sum_{i=2,3}\left(x_{i} / f-\partial_{i} f\right) \partial_{x_{i}} X_{j}-\partial_{1} f \partial_{x_{1}} X_{j}\right) \partial_{X_{j}} V \\
=\partial_{1} f\left(1-\partial_{1} \varphi_{-}\right)+\sum_{i=2,3}\left(x_{i} / f-\partial_{i} f\right) \partial_{i} \varphi_{-} .
\end{aligned}
$$

Here the variable $x=\left(x_{1}, x_{2}, x_{3}\right)$ is a function of $X=\left(X_{1}, X_{2}, X_{3}\right)$ and $V(X)$.

By the regularity theory of second order elliptic equations with cornered boundaries and the fact that the vector field $\partial_{\theta} \equiv X_{2} \partial_{X_{3}}-X_{3} \partial_{X_{2}}$ is simultaneously tangent to the surfaces $X_{1}=0, X_{1}=1$, and $X_{2}^{2}+X_{3}^{2}=1$, it is actually valid to assume more regularity of $V(X)$ with respect to $\theta$ by requiring

$$
\left\|\partial_{\theta} V\right\|_{C^{1,1-\delta_{0}}\left(\bar{Q}_{+}\right)} \leq C_{0} \varepsilon \quad \text { and } \sup _{X \in Q_{+}} R_{X}^{k-2+\delta_{0}}\left|\nabla_{X}^{k} \partial_{\theta} V\right| \leq C_{0} \varepsilon
$$

for $k=2,3$, where $R_{X}=X_{1}\left(1-X_{1}\right)+1-\left(X_{2}^{2}+X_{3}^{2}\right)$. For more details, see Lemma 5.3.

Next we turn to the nonexistence of solutions to the transonic shock problem with general given pressure $p\left(\tilde{\rho}_{+}(x)\right)$ at the exit of the nozzle.

Suppose that the nozzle wall is $C^{5}$-regular for $-1 \leq x_{1} \leq 1$ and that it consists of two surfaces $\Pi_{1}$ and $\Pi_{2}$, where $\Pi_{1}$ includes the converging part of the nozzle and $\Pi_{2}$ is a cone-shaped surface in $-1 / 2 \leq x_{1} \leq 1$ (that is, the widening part of the nozzle) whose vertex is $\left(x_{1}^{0}, 0,0\right)$ with $x_{1}^{0}<0$ and $\left|x_{1}^{0}\right|$ sufficiently large. Also suppose $\Pi_{1}$ and $\Pi_{2}$ are close to the cylindrical surface $\left\{x: x_{2}^{2}+x_{3}^{2}=1,-1 \leq x_{1} \leq\right.$ 1\}. More precisely, we assume that $\Pi_{2}$ is given by $x_{2}^{2}+x_{3}^{2}=\left(x_{1}-x_{1}^{0}\right)^{2} \operatorname{tg}^{2} \alpha_{0}\left(\alpha_{0}>\right.$ $0)$, where $\operatorname{tg} \alpha_{0}=1 /\left(1-x_{1}^{0}\right)$. Then $\Pi_{2}$ is close to the cylinder $x_{2}^{2}+x_{3}^{2}=1$ in $-1 / 2 \leq$ $x_{1} \leq 1$ for sufficiently large $\left|x_{1}^{0}\right|$, since $x_{2}^{2}+x_{3}^{2}=\left(x_{1}-x_{1}^{0}\right)^{2} /\left(1-x_{1}^{0}\right)^{2}$. In addition, we assume that the transonic shock goes through some fixed point $\tilde{x}_{0}=\left(\tilde{x}_{1}^{0}, \tilde{x}_{2}^{0}, \tilde{x}_{3}^{0}\right)$ at $\Pi_{2}$ with $r^{0}=\left(\left(\tilde{x}_{1}^{0}-x_{1}^{0}\right)^{2}+\left(\tilde{x}_{2}^{0}\right)^{2}+\left(\tilde{x}_{3}^{0}\right)^{2}\right)^{1 / 2}=-x_{1}^{0}$, and the supersonic incoming flow is symmetric in $-x_{1}^{0}-1 / 4 \leq r \leq-x_{1}^{0}$ with $r=\left(\left(x_{1}-x_{1}^{0}\right)^{2}+x_{2}^{2}+x_{3}^{2}\right)^{1 / 2}$ (that is, the potential $\varphi_{-}(x)$ depends only on $r$ for $-x_{1}^{0}-1 / 4 \leq r \leq-x_{1}^{0}$ ) and is a small perturbation of the constant state $\left(\rho_{0}, q_{-}, 0,0\right)$. By the hyperbolicity, one 
can obtain a supersonic flow $\varphi_{-}(x)$ in the global nozzle that which is symmetric in $-x_{1}^{0}-1 / 4 \leq r \leq\left(1-x_{1}^{0}\right) \sec \alpha_{0}$ and close to $q_{-} x_{1}$. Furthermore, the boundary condition (1-12) is replaced by

$$
H\left(C_{0}-1 / 2\left|\nabla \varphi_{+}\right|^{2}\right)=\rho_{+} \quad \text { on } r=\left(1-x_{1}^{0}\right) \sec \alpha_{0},
$$

where the constant density $\rho_{+}$is determined by (1-7).

To study the transonic problem with the boundary condition $(1-12)^{\prime}$ we may introduce as in Remarks 1.3 and 1.4 the partial hodograph transformation

$$
\begin{aligned}
& X_{1}=1-\frac{g\left(x_{2}, x_{3}\right)-x_{1}}{g\left(x_{2}, x_{3}\right)-x_{1}+\varphi_{-}(x)-\varphi_{+}(x)}, \\
& X_{i}=x_{i} / f(x) \quad \text { for } i=2,3
\end{aligned}
$$

with $g\left(x_{2}, x_{3}\right)=x_{1}^{0}+\left(\left(1-x_{1}^{0}\right)^{2} \sec ^{2} \alpha_{0}-\left(x_{2}^{2}+x_{3}^{2}\right)\right)^{1 / 2}$ and $f(x)=\left(x_{1}-x_{1}^{0}\right) \operatorname{tg} \alpha_{0}$. Under the transformation $(1-14)^{\prime}, \Omega_{+}$is changed onto

$$
Q_{+}=\left\{\left(X_{1}, X_{2}, X_{3}\right): 0<X_{1}<1, X_{2}^{2}+X_{3}^{2}<1\right\} .
$$

Define a new unknown function $W(X)=g\left(x_{2}, x_{3}\right)-x_{1}+\varphi_{-}(x)-\varphi_{+}(x)$. Then we proceed as in Remark 1.4: for any solution to the problem (1-3) with (1-8)-(1-11), $(1-12)^{\prime}$, and (1-13) in same regularity class as set forth in Theorem 1.1, one may also assume that $W(X)$ satisfies

$$
\left\|\left(X_{2} \partial_{X_{3}}-X_{3} \partial_{X_{2}}\right) W\right\|_{C^{1,1-\delta_{0}}\left(\bar{Q}_{+}\right)} \leq C_{0} \varepsilon,
$$

$$
\sup _{X \in Q_{+}} R_{X}^{k-2+\delta_{0}}\left|\nabla_{X}^{k}\left(X_{2} \partial_{X_{3}}-X_{3} \partial_{X_{2}}\right) W\right| \leq C_{0} \varepsilon \quad \text { for } k=2,3 \text {, }
$$

with $R_{X}=X_{1}\left(1-X_{1}\right)+1-\left(X_{2}^{2}+X_{3}^{2}\right)$.

Then from Theorem 1.1 and the assumption $(1-15)^{\prime}$, we can show the following nonexistence result.

Theorem 1.5 (nonexistence). If the nozzle wall consists of $\Pi_{1}$ and $\Pi_{2}$ as described above, then one can find supersonic incoming flows that are small perturbations of $\left(\rho_{0}, q_{-}, 0,0\right)$ such that the problem (1-3) with (1-8)-(1-11), (1-12)', and (1-13) has no transonic shock solution $\left(\varphi_{+}(x), \xi\left(x_{2}, x_{3}\right)\right)$ with the regularity and estimates stated in Theorem 1.1 and (1-15)'.

Remark 1.6. For the arbitrarily given and appropriately large pressure $p\left(\tilde{\rho}_{+}(x)\right)$ at the exit, Theorem 1.5 states that the transonic problem (1-3) in the nozzle with a shock can not occur. Moreover, it follows from the proof of Theorem 1.5 that the assumption (1-8) is actually not needed, that is, even if one adjusts the position of the possible shock at the widening part in $-1 / 4 \leq x_{1} \leq 1$, the transonic shock does not exist yet; see Remark 4.7. 
Remark 1.7. For complete Euler equations, if the widening part of the nozzle walls are straight and the corresponding supersonic incoming flow in the widening part is symmetric, then it can be proved that the transonic shock wave pattern can occur when the scope of pressure at the exit of the nozzle is appropriately given (it plays a crucial role in the proof that the entropies are different on two sides of the shock surface for the complete Euler system). The details can be found in [Xin and Yin 2005b; Xin et al. 2008].

Despite the nonexistence result in Theorem 1.5, we can find a class of pressures (although we do not give the pressure directly at the exit) that are induced by the oblique derivative boundary conditions (1-12)" so that the transonic shock problem (1-3) has a unique solution and satisfies the boundary conditions

$$
\partial_{1} \varphi_{+}+b_{2}(x) \partial_{2} \varphi_{+}+b_{3}(x) \partial_{3} \varphi_{+}+b_{1}(x) \varphi_{+}=g(x) \quad \text { on } x_{1}=1 .
$$

Here $b_{i}(x) \in C^{3}(\bar{\Omega})$ for $i=1,2,3 g(x) \in C^{3}(\bar{\Omega})$, and $\lambda \leq b_{1}\left(1, x_{2}, x_{3}\right) \leq \Lambda$ for $\left(x_{2}, x_{3}\right) \in\left\{\left(x_{2}, x_{3}\right):\left(x_{2}^{2}+x_{3}^{2}\right)^{1 / 2}=f\left(1, x_{2}, x_{3}\right)\right\}$, where $\Lambda$ and $\lambda$ are two positive constants. Also, $\left|\nabla^{\alpha}\left(g(x)-\left(1+b_{1}(x) x_{1}\right) q_{+}\right)\right|+\left|\nabla^{\alpha} b_{2}(x)\right|+\left|\nabla^{\alpha} b_{3}(x)\right| \leq \varepsilon$ for $0 \leq|\alpha| \leq 3$ and $x \in \bar{\Omega}$. With the same notations as in Theorem 1.1, the main existence result can be stated as follows:

Theorem 1.8. Let the assumptions (1-4), (1-5), and (1-7) hold. Then for suitably small $\varepsilon>0$, there exists a unique transonic pair $\left(\varphi(x), \xi\left(x_{2}, x_{3}\right)\right)$ such that $\varphi(x)$ is piecewise smooth, that is,

$$
\varphi(x)= \begin{cases}\varphi_{-}(x) & \text { if } x_{1}<\xi\left(x_{2}, x_{3}\right), \\ \varphi_{+}(x) & \text { if } x_{1}>\xi\left(x_{2}, x_{3}\right)\end{cases}
$$

and $\left(\varphi(x), \xi\left(x_{2}, x_{3}\right)\right)$ solve the problem (1-3), (1-9)-(1-11), (1-12)", and (1-13).

Moreover, for a given constant $0<\delta_{0}<1 / 3$, there exists a constant $C$ independent of $\varepsilon$ with the following properties.

(i) Regularity of supersonic flow: $\varphi_{-}(x) \in C^{5}(\bar{\Omega})$ solves the initial-boundary value problem (1-6) in $\Omega$. Also $\left\|\varphi_{-}(x)-q_{-} x_{1}\right\|_{C^{5}(\bar{\Omega})} \leq C \varepsilon$.

(ii) Regularity of the shock surface:

$$
\begin{array}{lr}
\xi\left(x_{2}, x_{3}\right) \in C^{1,1-\delta_{0}}(\bar{S}) \cap C^{3}(S), & \left\|\xi\left(x_{2}, x_{3}\right)\right\|_{C^{1,1-\delta_{0}(\bar{S})}} \leq C \varepsilon, \\
\left|\nabla_{x_{2}, x_{3}}^{k} \xi\left(x_{2}, x_{3}\right)\right| \leq C \varepsilon /\left|d_{\widetilde{\Gamma}_{1}}\right|^{k-2+\delta_{0}} & \text { for } k=2,3 \text { and }\left(x_{2}, x_{3}\right) \in S .
\end{array}
$$

(iii) Regularity of the subsonic flow: $\varphi_{+}(x) \in C^{1,1-\delta_{0}}\left(\bar{\Omega}_{+}\right) \cap C^{3}\left(\Omega_{+}\right)$admits the estimates $\left\|\varphi_{+}(x)-q_{+} x_{1}\right\|_{C^{1,1-\delta_{0}}\left(\bar{\Omega}_{+}\right)} \leq C \varepsilon$ and $\left|\nabla_{x}^{k} \varphi_{+}(x)\right| \leq C \varepsilon /\left|d_{x}\right|^{k-2+\delta_{0}}$ for $k=2,3$ and $x \in \Omega_{+}$.

(iv) Entropy condition: The entropy must satisfy the physical condition (1-11) on the shock $\Sigma$. 
Remark 1.9. It should be noted that the main assumption in Theorems $1.1,1.5$, and 1.8 is that the wall of the nozzle is a small perturbation (1-4) of a straight cylinder. This is in general necessary for the existence of such a transonic shock wave pattern. This is because for nozzles that deviate significantly from a flat cylinder, there may be supersonic shocks in the supersonic region, or supersonic bubbles surrounded by subsonic flow; see [Bers 1954; Courant and Friedrichs 1948].

Obviously, combining Theorem 1.1 with Theorem 1.8 yields the following result on the existence and uniqueness for a class of pressures at the exit of the nozzle.

Theorem 1.10 (Existence and uniqueness for a class of pressures at the exit). If $\tilde{\rho}_{+}\left(x_{2}, x_{3}\right)$ in (1-12) and a specified point in (1-8) are determined by Theorem 1.8, then the problem (1-3) with (1-8)-(1-13) has a unique transonic shock solution.

We now comment on the proof of the main results. Some of the main difficulties are that (1-3) is a mixed-type quasilinear equation and the shock surface is a free boundary with nonlinear boundary condition (1-10). In order to prove Theorem 1.1, the main strategy of the analysis comes from our treatment on the two-dimensional problem in [Xin and Yin 2005a]. First, we introduce a new partial hodograph transformation that maps the domain $\bar{\Omega}_{+}$into the fixed domain $\bar{Q}_{+}=\left\{\left(X_{1}, X_{2}, X_{3}\right): 0 \leq X_{1} \leq 1, X_{2}^{2}+X_{3}^{2} \leq 1\right\}$ as in [Xin and Yin 2005a]; see also [Chen et al. 2002; Majda and Thomann 1987; Meřrmanov 1980]. Under this transformation, the quasilinear potential Equation (1-3), whose coefficients contain only the first order derivatives of $\varphi(x)$, becomes a new second order nonlinear equation with coefficients and source term containing the unknown function $V(X)$ and its first order derivatives $\nabla_{X} V(X)$. Correspondingly, the boundary conditions (1-10)(1-13) are also changed into new nonlinear boundary conditions containing $V(X)$ and $\nabla_{X} V(X)$. It is crucial in our analysis that we can choose the partial-hodograph transformation so that the coefficients of $V(X)$ and $\nabla_{X} V(X)$ in the second order elliptic equation and the coefficients of $V(X)$ in the boundary conditions are all suitably small in an appropriately weighted space; we thus avoid the possible appearance of a negative eigenvalue for the resulting linear equation on $v(X)$. A key element in the proof of Theorem 1.1 is deriving $\|v(X)\|_{H^{2}}=0$ for the solution $v(X)$ by the multiplier method rather than establishing $\|v\|_{L^{\infty}}=0$ by the maximum principle, since it seems difficult [Lieberman and Trudinger 1986] to obtain $\|v\|_{L^{\infty}}$ by the maximum principle due to the structures of the equation and boundary conditions on $v(X)$. To prove Theorem 1.8, we will use the Schauder fixed point theorem to solve the corresponding nonlinear elliptic equation on $\bar{Q}_{+}$which is the result of the generalized hodograph transformation in Section 2. Weighted Hölder spaces will be used to treat the possible singularities due to the corners of the domain [Čanić et al. 2000; Gilbarg and Hörmander 1980; Lieberman and Trudinger 1986; Lieberman 1988; 1987; Xin and Yin 2005a]. In addition, one can use the maximum 
principle to derive the uniform $L^{\infty}$-estimate by use of (1-12)". Although the main strategy for proving Theorem 1.1 and Theorem 1.8 is similar to the approach we used for the two-dimensional case [Xin and Yin 2005a], much more delicate a priori estimates are needed to overcome certain difficulties occurring in the threedimensional case. In particular, more complicated and careful analyses are needed for the estimates near shock and fixed boundaries. Finally, based on Theorem 1.1 and making full use of the symmetric properties of nozzle wall $\Pi_{2}$, the supersonic incoming flow in the widening part for $-1 / 4 \leq x_{1} \leq 1 / 4$, and the end constant pressure, we can show that the pressure at the exit is uniquely determined by the supersonic incoming flow for the transonic solution with a shock. The main idea is that we can derive an ordinary differential equation using the symmetry of the nonlinear equation, the nozzle wall, and the boundary conditions, provided we assume that the transonic shock exists with respect to an appropriately large pressure at the exit. The reduction procedure is rather delicate and complicated but leads to the desired nonexistence result, Theorem 1.5.

Next, we note that there have been many studies on transonic problems, as we mentioned in [Xin and Yin 2005a]. See also [Alt et al. 1985; Čanić et al. 2000; Chen and Feldman 2003] and the references therein. In particular, we mention Alt, Caffarelli, and Friedman's study [1985] of the existence and uniqueness of axially symmetric compressible subsonic flows of jets and cavities. They use a variational approach to solve such a free boundary problem. However, it seems that we are not able to adapt their analysis because we have different conditions on both the free and fixed boundaries and because our problem is truly 3-dimensional.

The rest of the paper is organized as follows. In Section 2, we introduce a generalized partial hodograph transformation and reformulate the original problem (1-3) with the boundary conditions (1-9)-(1-13) in terms of the new variables. In addition, we carry out some basic estimates of the coefficients in the resulting problem. Section 3 derives the $H^{2}$-norm estimate for the solution $v(X)$ to the linear problem that emerged in Section 2. This directly yields $v(X) \equiv 0$, that is, it completes the proof of Theorem 1.1. In Section 4 we show with the help of Theorem 1.1 the nonexistence result in Theorem 1.5. Finally, Section 4 gives the proof of Theorem 1.8.

From now on, we conventionally take $O(\varepsilon)$ and $O(M \varepsilon)$ to mean that there is a generic constant $C$ such that $|O(\varepsilon)| \leq C \varepsilon$ and $|O(M \varepsilon)| \leq C M \varepsilon$, respectively, where $C$ is independent of $M$ and $\varepsilon$.

\section{The reformulation of Theorem 1.1 and the generalized hodograph transformation}

After determining $\varphi_{-}(x)$ by solving the initial-boundary value problem (1-6), 
the nonlinear mixed-type equation (1-3) with (1-8)-(1-13) can be reduced to a boundary value problem for a second order quasilinear elliptic equation with a free boundary (the transonic shock). In this section, we reduce this free boundary value problem on $\Omega_{+}$to a boundary value problem on a fixed domain $Q_{+}=$ $\left\{\left(X_{1}, X_{2}, X_{3}\right): 0<X_{1}<1, X_{2}^{2}+X_{3}^{2}<1\right\}$ by introducing a generalized hodograph transformation and a coordinate transformation. First, we estimate the potential $\varphi_{-}(x)$ for the supersonic flow.

Lemma 2.1. Assume that (1-4) and (1-5) hold. Then (1-6) has a unique solution $\varphi_{-}(x) \in C^{5}(\bar{\Omega})$ such that $\left\|\varphi_{-}(x)-q_{-} x_{1}\right\|_{C^{5}(\bar{\Omega})} \leq C \varepsilon$ for small $\varepsilon>0$, where $C$ is independent of $\varepsilon$.

Proof. Note that $\tilde{\varphi}(x)=\varphi_{-}(x)-q_{-} x_{1}$ satisfies

$$
\begin{aligned}
\left(\left(q_{-}+\partial_{1} \tilde{\varphi}\right)^{2}-c_{-}^{2}\right) \partial_{1}^{2} \tilde{\varphi}+2\left(q_{-}\right. & \left.+\partial_{1} \tilde{\varphi}\right) \sum_{i=2,3} \partial_{i} \tilde{\varphi} \partial_{1 i}^{2} \tilde{\varphi} \\
& +\sum_{i=2,3}\left(\left(\partial_{i} \tilde{\varphi}\right)^{2}-c_{-}^{2}\right) \partial_{i}^{2} \tilde{\varphi}+2 \partial_{2} \tilde{\varphi} \partial_{3} \tilde{\varphi} \partial_{23}^{2} \tilde{\varphi}=0,
\end{aligned}
$$

and

$$
\begin{aligned}
\left.\tilde{\varphi}(x)\right|_{x_{1}=-1}=\left.\partial_{1} \tilde{\varphi}(x)\right|_{x_{1}=-1} & =0, \\
\partial_{1} f \partial_{1} \tilde{\varphi}+\sum_{i=2,3}\left(\partial_{i} f-x_{i} / f\right) \partial_{i} \tilde{\varphi} & =-q_{-} \partial_{1} f \quad \text { on }\left(x_{2}^{2}+x_{3}^{2}\right)^{1 / 2}=f(x),
\end{aligned}
$$

where $c_{-}=c\left(H\left(C_{0}-\frac{1}{2}\left(\left|q_{-}+\partial_{1} \tilde{\varphi}\right|^{2}+\left|\partial_{2} \tilde{\varphi}\right|^{2}+\left|\partial_{3} \tilde{\varphi}\right|^{2}\right)\right)\right)$.

It follows from (1-5) that the initial-boundary values in (2-1) and (2-2) satisfy the compatibility conditions up to the $\left(k_{0}-1\right)$-st order. Since $q_{-}>c\left(\rho_{0}\right),(2-1)$ and (2-2) are strictly hyperbolic with respect to the $x_{1}$-direction for small perturbations of a constant solution. Using the standard energy estimate for the linear wave equation with the initial-boundary conditions and the Picard iteration (see for example [John 1990]), (2-1) and (2-2) have for small $\varepsilon>0$ the unique solution $\tilde{\varphi}(x) \in \bigcap_{i=0}^{k_{0}} C^{i}\left([-1,1], H^{k_{0}-i}(\bar{\Omega})\right)$, and there exists a constant $C$ independent of $\varepsilon$ such that

$$
\sum_{i=0}^{k_{0}}\|\tilde{\varphi}(x)\|_{C^{i}\left([-1,1], H^{k_{0}-i}(\bar{\Omega})\right)} \leq C \varepsilon .
$$

Hence the Sobolev's imbedding theorem implies that Lemma 2.1 holds.

We now reduce the free boundary value problem (1-3) with (1-8)-(1-13) to a fixed boundary value problem. Without loss of generality, we will henceforth assume that $q_{-}-q_{+}=1$ unless otherwise stated. Define new independent variables 
as

$$
X_{i}= \begin{cases}1-\frac{1-x_{1}}{1-x_{1}+\varphi_{-}(x)-\varphi_{+}(x)} & \text { if } i=1, \\ x_{i} / f(x) & \text { if } i=2,3 .\end{cases}
$$

It is expected that $\left|\partial_{x}^{\alpha}\left(\varphi_{+}(x)-q_{+} x_{1}\right)\right| \leq C \varepsilon$ for $0 \leq|\alpha| \leq 1$. Consequently, one has $\partial_{1}\left(\varphi_{-}(x)-\varphi_{+}(x)\right)=\partial_{1}\left(\varphi_{-}(x)-q_{-} x_{1}\right)-\partial_{1}\left(\varphi_{+}(x)-q_{+} x_{1}\right)+q_{-}-q_{+}>1 / 2$ for small $\varepsilon$ and all $x \in \Omega_{+}$. This implies $\varphi_{-}(x)>\varphi_{+}(x)$ when $x_{1}>\xi\left(x_{2}, x_{3}\right)$. It follows that (2-3) is an invertible transformation from the domain $\bar{\Omega}_{+}$to

$$
\bar{Q}_{+}=\left\{\left(X_{1}, X_{2}, X_{3}\right): 0 \leq X_{1} \leq 1, X_{2}^{2}+X_{3}^{2} \leq 1\right\} .
$$

Furthermore, the boundaries $x_{1}=\xi\left(x_{2}, x_{3}\right), x_{1}=1$, and $\left(x_{2}^{2}+x_{3}^{2}\right)^{1 / 2}=f(x)$ are transformed into $X_{1}=0, X_{1}=1$, and $X_{2}^{2}+X_{3}^{2}=1$, respectively.

Now, as in [Xin and Yin 2005a], we define a new unknown function

$$
V(X)=1-x_{1}+\varphi_{-}(x)-\varphi_{+}(x)
$$

One would expect that $V(X)=1+O(\varepsilon)$ and $\nabla_{X} V(X)=O(\varepsilon)$. These properties are important in the later analysis. It now follows from (2-3) and (2-4) that

$$
x_{i}= \begin{cases}1+\left(X_{1}-1\right) V(X) & \text { if } i=1, \\ x_{i}\left(1+\left(X_{1}-1\right) V(X), X_{2}, X_{3}\right) & \text { if } i=2,3 .\end{cases}
$$

Here $x_{i}\left(1+\left(X_{1}-1\right) V, X_{2}, X_{3}\right) \in C^{5}$ for $i=1,2$ on $X$ and $V$; this follows from the smoothness of $f(x)$ and the assumption (1-5). Direct calculations yield

$$
\partial_{x_{j}} X_{1}=D(X, V, \nabla V)\left(\delta_{i j}-\left(X_{1}-1\right) \sum_{i=2,3} \partial_{X_{i}} V \partial_{x_{j}} X_{i}\right) \quad \text { for } j=2,3,
$$

$$
\begin{aligned}
\partial_{x_{j}} X_{i} & =f^{-2}\left(f \delta_{i j}-x_{i} \partial_{x_{j}} f\right) \\
& =\delta_{i j}+O(\varepsilon) \quad \text { for } i=2,3 \text { and } j=1,2,3
\end{aligned}
$$

and

$$
\begin{aligned}
\partial_{x_{j} x_{k}}^{2} X_{1} & =\sum_{i, l=1,2,3} b_{i l}^{j k} \partial_{X_{i} X_{l}}^{2} V+b_{0}^{j k} \quad \text { for } j, k=1,2,3 \\
\partial_{x_{j} x_{k}}^{2} X_{i} & =-f^{-2}\left(\delta_{i j} \partial_{x_{k}} f+\delta_{i k} \partial_{x_{j}} f+x_{i} \partial_{x_{j} x_{k}}^{2} f\right)+2 f^{-3} x_{i} \partial_{x_{j}} f \partial_{x_{k}} f \\
& =O(\varepsilon) \quad \text { for } i=2,3 \text { and } j, k=1,2,3
\end{aligned}
$$

where

$$
D(X, V, \nabla V)=\left(V+\left(X_{1}-1\right) \partial_{X_{1}} V\right)^{-1},
$$


and

$$
\begin{aligned}
& b_{i l}^{j k} \equiv b_{i l}^{j k}(X, V, \nabla V) \\
&= \frac{1}{2} D(X, V, \nabla V)\left(1-X_{1}\right)\left(\partial_{x_{k}} X_{l} \partial_{x_{j}} X_{i}+\partial_{x_{k}} X_{i} \partial_{x_{j}} X_{l}\right), \\
& b_{0}^{j k} \equiv b_{0}^{j k}(X, V, \nabla V) \\
&=-D(X, V, \nabla V)\left(2 \partial_{x_{k}} X_{1} \partial_{x_{j}} X_{1}+\partial_{x_{k}} X_{2} \partial_{x_{j}} X_{1}+\partial_{x_{j}} X_{2} \partial_{x_{k}} X_{1}\right. \\
& \quad+\left(X_{1}-1\right) \partial_{x_{j} x_{k}}^{2} X_{2}+\partial_{x_{j}} X_{3} \partial_{x_{k}} X_{1} \\
&\left.\quad+\partial_{x_{k}} X_{3} \partial_{x_{j}} X_{1}+\left(X_{1}-1\right) \partial_{x_{j} x_{k}}^{2} X_{3}\right) \nabla_{X} V .
\end{aligned}
$$

We note that for suitably small $\varepsilon$, the functions $D(X, V, \nabla V), b_{i l}^{j k}(X, V, \nabla V)$ and $b_{0}^{j k}(X, V, \nabla V)$ are all smooth functions of $X, V$, and $\nabla_{X} V$.

In terms of the new variables (2-3) and (2-4), Equation (1-3) becomes

$$
\sum_{i, j=1,2,3} a_{i j}\left(X, V, \nabla_{X} V\right) \partial_{X_{i} X_{j}}^{2} V+F_{0}\left(X, V, \nabla_{X} V\right)=0
$$

where

$$
\begin{aligned}
a_{i j}\left(X, V, \nabla_{X} V\right)= & \sum_{k, l=1,2,3} \tilde{a}_{k l}\left(\varphi_{+}\right)\left(\partial_{x_{k}} X_{i} \partial_{x_{l}} X_{j}+b_{i j}^{k l} \partial_{X_{1}} V\right), \\
F_{0}\left(X, V, \nabla_{X} V\right)= & \sum_{i, k=1,2,3} \tilde{a}_{i k}\left(\varphi_{+}\right)\left(b_{0}^{i k} \partial_{X_{1}} V+\sum_{j=2,3} \partial_{X_{j}} V \partial_{x_{i} x_{k}}^{2} X_{j}\right) \\
& -\sum_{i, k=1,2,3}\left(\tilde{a}_{i k}\left(\varphi_{+}\right)-\tilde{a}_{i k}\left(\varphi_{-}\right)\right) \partial_{x_{i} x_{k}}^{2} \varphi_{-} .
\end{aligned}
$$

Here the matrix $\left(\tilde{a}_{i k}\right)$ is defined by

$$
\tilde{a}_{i j}(\varphi)=\left(\begin{array}{ccc}
\left(\partial_{x_{1}} \varphi\right)^{2}-C^{2}(\nabla \varphi) & \partial_{x_{1}} \varphi \partial_{x_{2}} \varphi & \partial_{x_{1}} \varphi \partial_{x_{3}} \varphi \\
\partial_{x_{1}} \varphi \partial_{x_{2}} \varphi & \left(\partial_{x_{2}} \varphi\right)^{2}-C^{2}(\nabla \varphi) & \partial_{x_{2}} \varphi \partial_{x_{3}} \varphi \\
\partial_{x_{1}} \varphi \partial_{x_{3}} \varphi & \partial_{x_{2}} \varphi \partial_{x_{3}} \varphi & \left(\partial_{x_{3}} \varphi\right)^{2}-C^{2}(\nabla \varphi)
\end{array}\right)_{i j}
$$

with sound speed $C(\nabla \varphi)=c\left(H\left(C_{0}-\frac{1}{2}|\nabla \varphi|^{2}\right)\right)$. In deriving (2-9) from (1-3), we have used the Equation (1-6). It is important to note that the quasilinear Equation (2-9) is uniformly elliptic in $Q_{+}$provided that $\varepsilon$ is suitably small.

This and other important properties of $a_{i j}\left(X, V, \nabla_{X} V\right)$ and $F_{0}\left(X, V, \nabla_{X} V\right)$ will be listed in Lemma 2.4 below.

Next, we transform the boundary conditions in terms of the new variables. First, it follows from (1-9) that the Rankine-Hugoniot condition (1-10) is equivalent to

$$
\sum_{i=1,2,3}\left[\partial_{i} \varphi H\right] \partial_{i}\left(\varphi_{+}-\varphi_{-}\right)=0 \quad \text { on } x_{1}=\xi\left(x_{2}, x_{3}\right),
$$


which in the new coordinates takes the form

$$
G\left(X, V, \nabla_{X} V\right)=0 \quad \text { on } X_{1}=0,
$$

where

$$
\begin{aligned}
G\left(X, V, \nabla_{X} V\right)=H( & \left.C_{0}-\frac{1}{2}\left(\left(1+\partial_{x_{1}} V-\partial_{1} \varphi_{-}\right)^{2}+\sum_{i=2,3}\left(\partial_{x_{i}} V-\partial_{i} \varphi_{-}\right)^{2}\right)\right) \\
\times & \left(\left(\partial_{1} \varphi_{-}-\partial_{x_{1}} V-1\right)\left(1+\partial_{x_{1}} V\right)+\sum_{i=2,3}\left(\partial_{i} \varphi_{-}-\partial_{x_{i}} V\right) \partial_{x_{i}} V\right) \\
& \quad\left(\partial_{1} \varphi_{-}\left(1+\partial_{x_{1}} V\right)+\sum_{i=2,3} \partial_{i} \varphi_{-} \partial_{x_{i}} V\right) H\left(C_{0}-\frac{1}{2}\left|\nabla \varphi_{-}\right|^{2}\right) .
\end{aligned}
$$

Analogously, (1-12) and (1-13) are transformed respectively into

$$
H\left(C_{0}-\frac{1}{2}\left(\left(1+\partial_{x_{1}} V-\partial_{1} \varphi_{-}\right)^{2}+\sum_{i=2,3}\left(\partial_{x_{i}} V-\partial_{i} \varphi_{-}\right)^{2}\right)\right)=\tilde{\rho}_{+}(x)
$$

on $X_{1}=1$ and, on $X_{2}^{2}+X_{3}^{2}=1$,

$$
\begin{aligned}
\sum_{j=1,2,3}\left(\sum_{i=2,3}\left(x_{i} / f-\partial_{i} f\right) \partial_{x_{i}} X_{j}\right. & \left.-\partial_{1} f \partial_{x_{1}} X_{j}\right) \partial_{X_{j}} V \\
& =\partial_{1} f\left(1-\partial_{1} \varphi_{-}\right)+\sum_{i=2,3}\left(x_{i} / f-\partial_{i} f\right) \partial_{i} \varphi_{-}
\end{aligned}
$$

Here the variable $x=\left(x_{1}, x_{2}, x_{3}\right)$ is a function of $X=\left(X_{1}, X_{2}, X_{3}\right)$ and $V(X)$. It will be clear that (2-11) and (2-12) represent nonlinear uniform oblique derivative boundary conditions for (2-9).

Finally, it follows from (1-8) and the transformation (2-3) that

$$
V\left(\widetilde{X}^{0}\right)=1,
$$

where $\widetilde{X}^{0}=\left(\tilde{X}_{1}^{0}, \widetilde{X}_{2}^{0}, \widetilde{X}_{3}^{0}\right)=\left(0, \tilde{x}_{2}^{0} / f\left(\tilde{x}^{0}\right), \tilde{x}_{3}^{0} / f\left(\tilde{x}^{0}\right)\right)$.

Hence the major problem is reduced to studying the quasilinear Equation (2-9) on the domain $Q_{+}$with nonlinear boundary conditions (2-11)-(2-14).

It follows from the assumptions in Theorem 1.1 that $V(X) \in C^{1,1-\delta_{0}}\left(\bar{Q}_{+}\right) \cap$ $C^{3}\left(\bar{Q}_{+} \backslash \bigcup_{i=1}^{2} \Gamma_{i}\right)$ such that

(2-15) $\|V(X)-1\|_{C^{1,1-\delta_{0}}\left(\bar{Q}_{+}\right)} \leq C \varepsilon, \quad\left|\nabla_{X}^{k} V(X)\right| \leq \frac{C \varepsilon}{\left|R_{X}\right|^{k-2+\delta_{0}}} \quad$ for $k=2,3$,

where

$$
\begin{aligned}
\Gamma_{1} & =\left\{\left(0, X_{2}, X_{3}\right): X_{2}^{2}+X_{3}^{2}=1\right\}, \\
\Gamma_{2} & =\left\{\left(1, X_{2}, X_{3}\right): X_{2}^{2}+X_{3}^{2}=1\right\}, \\
R_{X} & =X_{1}\left(1-X_{1}\right)+1-\left(X_{2}^{2}+X_{3}^{2}\right) .
\end{aligned}
$$


Next we examine the nonlinear boundary conditions (2-11)-(2-13) so that we can treat the uniqueness result in Theorem 1.1.

Consider the boundary condition for $V$ on $X_{1}=0$ first. Note that the boundary condition (2-11) can be rewritten on $X_{1}=0$ as

$$
\sum_{i=1,2,3} B_{1 i}\left(X, V, \nabla_{X} V\right) \partial_{X_{i}} V+B_{1}\left(X, V, \nabla_{X} V\right)(V-1)=-G(X, 1,0,0,0),
$$

with

$$
\begin{aligned}
B_{1 i}\left(X, V, \nabla_{X} V\right) & =\int_{0}^{1} \partial_{\partial_{X_{i}} V} G\left(X, \theta(V-1)+1, \theta \nabla_{X} V\right) d \theta \quad \text { for } i=1,2,3, \\
B_{1}\left(X, V, \nabla_{X} V\right) & =\int_{0}^{1} \partial_{V} G\left(X, \theta(V-1)+1, \theta \nabla_{X} V\right) d \theta
\end{aligned}
$$

Due to (2-15), the following estimates hold for the coefficients of (2-16)

Lemma 2.2. We have

$$
\sum_{k=0, \ldots, 3}\left|\nabla_{X}^{k} G(X, 1,0,0,0)\right| \leq C \varepsilon
$$

and

$(2-18) \quad B_{11}\left(X, V, \nabla_{X} V\right)=-\rho_{+}\left(q_{+}-q_{-}\right)\left(c^{2}\left(\rho_{+}\right)-q_{+}^{2}\right) / c^{2}\left(\rho_{+}\right)(1+O(\varepsilon))$,

(2-19) $\quad B_{1 i}\left(X, V, \nabla_{X} V\right)=O(\varepsilon) \quad$ for $i=2,3$,

(2-20) $\quad B_{1}\left(X, V, \nabla_{X} V\right)=O(\varepsilon)$,

and, for $k=1,2$,

$$
\sum_{i=1}^{2}\left|\nabla_{X}^{k} B_{1 i}\left(X, V, \nabla_{X} V\right)\right|+\left|\nabla_{X}^{k} B_{1}\left(X, V, \nabla_{X} V\right)\right|=O\left(\frac{\varepsilon}{R^{k-1+\delta_{0}}}\right) .
$$

The proof of this lemma is given in the Appendix.

It follows from $q_{+}<c\left(\rho_{+}\right), q_{+}<q_{-}$, and (2-18) that $B_{11}\left(X, V, \nabla_{X} V\right) \neq 0$ for small $\varepsilon$. Thus one can rewrite (2-16) as

$$
\begin{aligned}
\partial_{X_{1}} V+\sum_{i=2,3} \widetilde{B}_{1 i}\left(X, V, \nabla_{X} V\right) \partial_{X_{i}} V+\widetilde{B}_{1}\left(X, V, \nabla_{X} V\right)(V-1) \\
=\widetilde{B}_{10}\left(X, V, \nabla_{X} V\right) \text { on } X_{1}=0
\end{aligned}
$$

where the coefficients satisfy the estimates

$$
\begin{aligned}
\widetilde{B}_{1 i}\left(X, V, \nabla_{X} V\right) & =O(\varepsilon), \quad \nabla_{X}^{k} \widetilde{B}_{1 i}\left(X, V, \nabla_{X} V\right)=O\left(\varepsilon / R^{k-1+\delta_{0}}\right), \\
\widetilde{B}_{1}\left(X, V, \nabla_{X} V\right) & =O(\varepsilon), \quad \nabla_{X}^{k} \widetilde{B}_{1}\left(X, V, \nabla_{X} V\right)=O\left(\varepsilon / R^{k-1+\delta_{0}}\right),
\end{aligned}
$$

for $k=1,2$ and $i=0,2,3$. These follow from Lemma 2.2.

Next we determine the boundary condition for $V(X)$ on $X_{1}=1$. 
Set

$$
\begin{array}{r}
\widetilde{G}\left(X, V, \nabla_{X} V\right)=H\left(C_{0}-\frac{1}{2}\left(\left(1+\partial_{x_{1}} V-\partial_{1} \varphi_{-}\right)^{2}+\sum_{i=2,3}\left(\partial_{x_{i}} V-\partial_{i} \varphi_{-}\right)^{2}\right)\right) \\
-H\left(C_{0}-\frac{1}{2} q_{+}^{2}\right) .
\end{array}
$$

Because $H\left(C_{0}-\frac{1}{2} q_{+}^{2}\right)=\rho_{+},(2-12)$ becomes $\widetilde{G}\left(X, V, \nabla_{X} V\right)=\tilde{\rho}_{+}(x)-\rho_{+}$on $X_{1}=1$. As in (2-22), a direct computation yields

$$
\begin{aligned}
\partial_{X_{1}} V+\sum_{i=2,3} \widetilde{B}_{2 i}(X, V, & \left.\nabla_{X} V\right) \partial_{X_{i}} V+\widetilde{B}_{2}\left(X, V, \nabla_{X} V\right)(V-1) \\
& =\widetilde{B}_{20}\left(X, V, \nabla_{X} V\right)\left(\tilde{\rho}_{+}(x)-\rho_{+}\right) \quad \text { on } X_{1}=1,
\end{aligned}
$$

where

$$
\begin{aligned}
\widetilde{B}_{2 i}\left(X, V, \nabla_{X} V\right) & =O(\varepsilon), & \nabla_{X}^{k} \widetilde{B}_{2 i}\left(X, V, \nabla_{X} V\right) & =O\left(\varepsilon / R^{k-1+\delta_{0}}\right), \\
\widetilde{B}_{2}\left(X, V, \nabla_{X} V\right) & =O(\varepsilon), & \nabla_{X}^{k} \widetilde{B}_{2}\left(X, V, \nabla_{X} V\right) & =O\left(\varepsilon / R^{k-1+\delta_{0}}\right)
\end{aligned}
$$

for $k=1,2$ and $i=0,2,3$.

Similarly, we can obtain the boundary condition for $V(X)$ on $X_{2}^{2}+X_{3}^{2}=1$ from

$$
\sum_{i=1,2,3} \widetilde{B}_{3 i}\left(X, V, \nabla_{X} V\right) \partial_{X_{i}} V+\tilde{B}_{3}(X, V)(V-1)=0 \quad \text { on } X_{2}^{2}+X_{3}^{2}=1
$$

The properties of $\widetilde{B}_{3 i}$ and $\widetilde{B}_{3}$ are described by the following lemma, whose proof we omit.

Lemma 2.3. Under the assumptions in (2-15), we have

$$
\begin{aligned}
\widetilde{B}_{31}\left(X, V, \nabla_{X} V\right) & =O(\varepsilon), & \nabla_{X}^{k} \widetilde{B}_{31}\left(X, V, \nabla_{X} V\right) & =O\left(\varepsilon / R^{k-1+\delta_{0}}\right), \\
\widetilde{B}_{3 i}\left(X, V, \nabla_{X} V\right)-X_{i} & =O(\varepsilon), & \nabla_{X}^{k}\left(\widetilde{B}_{3 i}\left(X, V, \nabla_{X} V\right)-X_{i}\right) & =O\left(\varepsilon / R^{k-1+\delta_{0}}\right),
\end{aligned}
$$

for $k=1,2$ and $i=2,3$. Also

$$
\nabla_{X}^{k} \widetilde{B}_{3}(X, V)= \begin{cases}O(\varepsilon) & \text { if } k=0,1 \\ O\left(\varepsilon / R^{k-2+\delta_{0}}\right) & \text { if } k=2,3\end{cases}
$$

In addition, we need more information on $a_{i j}\left(X, V, \nabla_{X} V\right)$ and $F_{0}\left(X, V, \nabla_{X} V\right)$. In the following lemma, we list some important estimates of $a_{i j}$ and $F_{0}$ which will be used later. 
Lemma 2.4. It follows from (2-15) that

$$
\begin{aligned}
a_{11}\left(X, V, \nabla_{X} V\right) & =\left(q_{+}^{2}-c_{+}^{2}\right)(1+O(\varepsilon)), & & \\
a_{i j}\left(X, V, \nabla_{X} V\right) & =O(\varepsilon), & & \text { for } 1 \leq i<j \leq 3, \\
a_{i i}\left(X, V, \nabla_{X} V\right) & =-c_{+}^{2}(1+O(\varepsilon)) & & \text { for } i=2,3, \\
F_{0}\left(X, V, \nabla_{X} V\right) & =O(\varepsilon), & & \\
\nabla_{X}^{k} a_{i j}\left(X, V, \nabla_{X} V\right) & =O\left(\varepsilon / R^{k-1+\delta_{0}}\right) & & \text { for } k=1,2, \\
\nabla_{X}^{k} F_{0}\left(X, V, \nabla_{X} V\right) & =O\left(\varepsilon / R^{k-1+\delta_{0}}\right) & & \text { for } k=1,2 .
\end{aligned}
$$

The proof of this lemma is sketched in the Appendix.

So far we have outlined the linearization of the Equation (2-9) and the boundary conditions (2-11)-(2-13) and derived some estimates on the corresponding coefficients. In the subsequent section, we will focus on the uniqueness of the solution to the problem (2-9) with (2-22), (2-23), (2-24), and (2-14).

\section{The proof of uniqueness}

In this section, we will use the preparations of Section 2 to prove Theorem 1.1. Suppose that there are two solutions $\left(\varphi_{+}^{1}(x), \xi^{1}\left(x_{2}, x_{3}\right)\right)$ and $\left(\varphi_{+}^{2}(x), \xi^{2}\left(x_{2}, x_{3}\right)\right)$ to the Equation (1-3) with (1-8)-(1-13) and that these satisfy the corresponding regularity conditions in Theorem 1.1. Then through the general partial hodograph transformation (2-3), we get two corresponding solutions $V_{1}(X)$ and $V_{2}(X)$ to Equation (2-9) with the boundary conditions (2-22), (2-23), (2-24), and (2-14). Moreover, $V_{j}(X) \in C^{1,1-\delta_{0}}\left(\bar{Q}_{+}\right) \cap C^{3}\left(\bar{Q}_{+} \backslash \bigcup_{i=1}^{2} \Gamma_{i}\right)$ for $j=1,2$ and satisfy the estimates in (2-15). Our aim is to prove $V_{1}(X) \equiv V_{2}(X)$ in $\bar{Q}_{+}$.

Set $v(X)=V_{1}(X)-V_{2}(X)$. Then it follows from the Equation (2-9) with (2-22), (2-23), (2-24), and (2-14) that

$$
\begin{cases}\sum_{i, j=1,2,3} a_{i j}\left(X, V_{1}, \nabla_{X} V_{1}\right) \partial_{X_{i} X_{j}}^{2} v & \\ \quad+\sum_{i=1,2,3} b_{i}(X) \partial_{X_{i}} v+c(X) v=0 & \text { for } X \in Q_{+}, \\ \partial_{X_{1}} v+\sum_{i=2,3} \gamma_{1 i}(X) \partial_{X_{i}} v+d_{1}(X) v=0 & \text { on } X_{1}=0, \\ \partial_{X_{1}} v+\sum_{i=2,3} \gamma_{2 i}(X) \partial_{X_{i}} v+d_{2}(X) v=0 & \text { on } X_{1}=1, \\ \sum_{i=1,2,3} \gamma_{3 i}(X) \partial_{X_{i}} v+d_{3}(X) v=0 & \text { on } X_{2}^{2}+X_{3}^{2}=1, \\ v\left(\widetilde{X}^{0}\right)=0 . & \end{cases}
$$

Due to (2-15), Lemmas 2.1-2.4, and the assumption on $\tilde{\rho}_{+}(x)$, we have the following estimates for $b_{i}(X), c(X)$, and $\gamma_{i j}(X), d_{i}(X)$. 
Lemma 3.1. The functions $b_{i}(X), c(X)$ lie in $C^{1}\left(\bar{Q}_{+} \backslash \bigcup_{i=1}^{2} \Gamma_{i}\right)$, and $\gamma_{i j}(X), d_{i}(X)$ lie in $C^{1}\left(\bar{Q}_{+} \backslash \bigcup_{i=1}^{2} \Gamma_{i}\right) \cap C^{1-\delta_{0}}\left(\bar{Q}_{+}\right)$. They satisfy the estimates

$$
\begin{aligned}
\sum_{i=1,2,3}\left|\nabla_{X}^{k} b_{i}(X)\right|+\left|\nabla_{X}^{k} c(X)\right| \leq C \varepsilon / R^{k+\delta_{0}} & \text { for } k=0,1, \\
\sum_{j}\left\|\gamma_{i j}\right\|_{C^{1-\delta_{0}}}+\sum_{j=1,2,3}\left\|d_{j}\right\|_{C^{1-\delta_{0}}} \leq C \varepsilon & \text { for } i=1,2, \\
\sum_{j=2,3}\left\|\gamma_{3 j}-X_{j}\right\|_{C^{1-\delta_{0}}}+\left\|\gamma_{31}\right\|_{C^{1-\delta_{0}}} \leq C \varepsilon, & \\
\sum_{j}\left|\nabla_{X}^{k} \gamma_{i j}\right|+\sum_{j=1,2,3}\left|\nabla_{X}^{k} d_{j}\right| \leq C \varepsilon / R^{k-1+\delta_{0}} & \text { for } i=1,2 \text { and } k=1,2, \\
\sum_{j=2,3}\left|\nabla_{X}^{k}\left(\gamma_{3 j}-X_{j}\right)\right|+\left|\nabla_{X}^{k} \gamma_{31}\right| \leq C \varepsilon / R^{k-1+\delta_{0}} & \text { for } k=1,2 .
\end{aligned}
$$

As noted in [Xin and Yin 2005a, Section 4], since $c(X)$ and $d_{i}(X)$ for $i=1,2,3$ can change their signs and $c(X)$ has singularities (that is, $|c(X)| \rightarrow \infty$ as $X_{1} \rightarrow 0$ or $X_{1} \rightarrow 1$ ), it seems difficult to derive $v \equiv 0$ by directly applying the maximum principle. Thus, we intend to establish $\|v\|_{H^{2}\left(Q_{+}\right)}=0$ by energy estimates. To this end, we first need an inequality of Poincaré type.

Lemma 3.2. If $u(X) \in H^{2}\left(Q_{+}\right)$and $u\left(\widetilde{X}^{0}\right)=0$, then there exists a constant $C$ independent of $u$ such that

$$
\int_{Q_{+}}|u|^{2} d X \leq C \int_{Q_{+}}\left(|\nabla u|^{2}+\left|\nabla^{2} u\right|^{2}\right) d X .
$$

Proof. This lemma can be proved by a technique in [Maz'ja 1985].

Indeed, if (3-2) does not hold, then for each $m \in \mathbb{N}$, there exists a function $u_{m} \in H^{2}\left(Q_{+}\right)$with $u_{m}\left(\tilde{X}^{0}\right)=0$ such that

$$
\int_{Q_{+}}\left|u_{m}\right|^{2} d X>m \int_{Q_{+}}\left(\left|\nabla u_{m}\right|^{2}+\left|\nabla^{2} u_{m}\right|^{2}\right) d X .
$$

Let $v_{m}=u_{m} /\left\|u_{m}\right\|_{L^{2}\left(Q_{+}\right)}$. Then $v_{m}$ has the properties
(i) $\left\|v_{m}\right\|_{L^{2}\left(Q_{+}\right)}=1$;
(iii) $v_{m} \in H^{2}\left(Q_{+}\right)$;$$
v_{m}\left(\widetilde{X}^{0}\right)=0 ;
$$$$
\text { (iv) } \int_{Q_{+}}\left(\left|\nabla v_{m}\right|^{2}+\left|\nabla^{2} v_{m}\right|^{2}\right) d X<1 / m \text {. }
$$

If follows from (i) and (iv) that there exist a subsequence $\left\{v_{m_{j}}\right\} \subset\left\{v_{m}\right\}$ and a function $v \in H^{2}\left(Q_{+}\right)$such that $v_{m_{j}} \rightarrow v$ in $H^{2}\left(Q_{+}\right)$. Then (iv) implies that $v=C$ for almost all $X \in Q_{+}$. In addition, $v_{m_{j}} \rightarrow v$ in $H^{2}\left(Q_{+}\right)$implies $v_{m_{j}} \rightarrow v$ in $C\left(\bar{Q}_{+}\right)$. Thus $v\left(\widetilde{X}^{0}\right)=0$, and so $v \equiv 0$. But this contradicts $\|v\|_{L^{2}\left(Q_{+}\right)}=1$. 
Lemma 3.3. Let $v$ be a solution to the problem (3-1). If $v$ has the regularities and estimates of (2-15), then, for suitably small $\varepsilon$, we have $v(X) \equiv 0$.

Proof. The proof will be divided into three steps.

Step 1: Estimate of $\|\nabla v\|_{L^{2}\left(Q_{+}\right)}$. Multiplying $v$ on both sides of (3-1) and integrating by parts on $Q_{+}$, we get

$$
-\int_{Q_{+}} \sum_{i, j=1,2,3} a_{i j} \partial_{i} v \partial_{j} v d X=\sum_{i=1}^{5} I_{5},
$$

where

$$
\begin{array}{ll}
I_{1}=\int_{Q_{+}} \sum_{i, j=1,2,3} \partial_{i} a_{i j} \partial_{j} v v d X, & I_{4}=-\int_{X_{2}^{2}+X_{3}^{2}=1} \sum_{i=2,3}\left(\sum_{j=1,2,3} a_{i j} \partial_{j} v v\right) X_{i} d S, \\
I_{2}=\int_{X_{1}=0} \sum_{j=1,2,3} a_{1 j} \partial_{j} v v d S, & I_{5}=-\int \sum_{Q_{+}}\left(\sum_{i=1,2,3} b_{i}(X) \partial_{i} v+c(X) v\right) v d X . \\
I_{3}=-\int_{X_{1}=1} \sum_{j=1,2,3} a_{1 j} \partial_{j} v v d S, &
\end{array}
$$

We treat the $I_{i}$ individually.

(i) Estimate of $I_{1}$.

For a small constant $\delta>0$, there exists a constant $C_{\delta}>0$ such that

$$
\left|\sum_{i, j=1,2,3} \partial_{i} a_{i j} \partial_{j} v v\right| \leq \delta|\nabla v|^{2}+C_{\delta} \sum_{i, j=1,2,3}\left|\nabla a_{i j}\right|^{2}|v|^{2}
$$

Hence by Lemma 2.4, Sobolev's imbedding theorem, and Lemma 3.2, we have

$$
\begin{aligned}
\left|I_{1}\right| & \leq \delta \int_{Q_{+}}|\nabla v|^{2} d X+C_{\delta} \varepsilon^{2}\left(\int_{Q_{+}}|v|^{4} d X\right)^{1 / 2} \\
& \leq \delta \int_{Q_{+}}|\nabla v|^{2} d X+C_{\delta} \varepsilon^{2} \int_{Q_{+}}\left(|\nabla v|^{2}+\left|\nabla^{2} v\right|^{2}\right) d X .
\end{aligned}
$$

(ii) Estimate of $I_{2}$ and $I_{3}$.

We only estimate $I_{2}$; the estimate of $I_{3}$ is similar. Decompose $I_{2}$ as $I_{2}=I_{21}+I_{22}$, where

$$
I_{21}=\int_{X_{1}=0} a_{11} \partial_{1} v v d S \quad \text { and } \quad I_{22}=\int_{X_{1}=0} \sum_{j=2,3} a_{1 j} \partial_{j} v v d S
$$


By the boundary conditions in (3-1), the trace theorem, Lemma 3.1, and Lemma 3.2, we have

$$
\left|I_{21}\right| \leq C \varepsilon \int_{X_{1}=0}\left(|v|^{2}+|\nabla v|^{2}\right) d S \leq C \varepsilon \int_{Q_{+}}\left(|\nabla v|^{2}+\left|\nabla^{2} v\right|^{2}\right) d X .
$$

A similar estimate holds for $I_{22}$ due to Lemma 2.4. Thus

$$
\left|I_{2}\right| \leq C \varepsilon \int_{Q_{+}}\left(|\nabla v|^{2}+\left|\nabla^{2} v\right|^{2}\right) d X .
$$

(iii) Estimate of $I_{4}$.

It follows from the boundary conditions in (3-1), Lemma 2.3, and Lemma 2.4 that

$$
\begin{aligned}
&\left|I_{4}\right| \leq \int_{X_{2}^{2}+X_{3}^{2}=1}\left|X_{2} \sum_{j \neq 2} a_{2 j} \partial_{j} v+X_{3} \sum_{j \neq 3} a_{3 j} \partial_{j} v\right||v| d S \\
&+\int_{X_{2}^{2}+X_{3}^{2}=1}\left|\left(a_{22}+c_{+}^{2}\right) X_{2} \partial_{2} v+\left(a_{33}+c_{+}^{2}\right) X_{3} \partial_{3} v\right||v| d S \\
&+c_{+}^{2} \int_{X_{2}^{2}+X_{3}^{2}=1}\left|\left(\gamma_{32}-X_{2}\right) \partial_{2} v+\left(\gamma_{33}-X_{3}\right) \partial_{3} v\right||v| d S \\
&+c_{+}^{2} \int_{X_{2}^{2}+X_{3}^{2}=1}\left|\gamma_{31} \partial_{1} v+d_{3} v\right||v| d S \\
& \leq C \varepsilon \int_{X_{2}^{2}+X_{3}^{2}=1}\left(|v|^{2}+|\nabla v|^{2}\right) d S .
\end{aligned}
$$

Hence by the trace theorem and Lemma 3.2, we get

$$
\left|I_{4}\right| \leq C \varepsilon \int_{Q_{+}}\left(|\nabla v|^{2}+\left|\nabla^{2} v\right|^{2}\right) d X .
$$

(iv) Estimate of $I_{5}$.

$$
\left|I_{5}\right| \leq C \varepsilon \int_{Q_{+}} \frac{(|\nabla v|+|v|)|v|}{R^{\delta_{0}}} d X \leq C \varepsilon \int_{Q_{+}}\left(|\nabla v|^{2}+\left|\nabla^{2} v\right|^{2}\right) d X .
$$

Substituting all the above estimates of the $I_{i}$ into (3-3), we obtain for small $\delta>0$ and $\varepsilon>0$ that

$$
\int_{Q_{+}}|\nabla v|^{2} d X \leq C \varepsilon \int_{Q_{+}}\left|\nabla^{2} v\right|^{2} d X .
$$

Step 2: Estimate of $\left\|\nabla \partial_{1} v\right\|_{L^{2}\left(Q_{+}\right)}$. Set $w_{i}=\partial_{i} v$ for $i=1,2,3$. Then $w_{1}$ satisfies

$$
\sum_{i, j=1,2,3} a_{i j} \partial_{i j}^{2} w_{1}+\sum_{i, j=1,2,3} \partial_{1} a_{i j} \partial_{i} w_{j}+\partial_{1}\left(\sum_{i=1,2,3} b_{i}(X) \partial_{X_{i}} v+c(X) v\right)=0
$$


and

$$
\begin{array}{ll}
w_{1}+\sum_{i=2,3} \gamma_{1 i} w_{i}+d_{1} v=0 & \text { on } X_{1}=0, \\
w_{1}+\sum_{i=2,3} \gamma_{2 i} w_{i}+d_{2} v=0 & \text { on } X_{1}=1,
\end{array}
$$

$$
\sum_{i=1,2,3} \gamma_{3 i} \partial_{i} w_{1}+\sum_{i=1,2,3} \partial_{1} \gamma_{3 i} w_{i}+d_{3} w_{1}+\partial_{1} d_{3} v=0 \quad \text { on } X_{2}^{2}+X_{3}^{2}=1 .
$$

Multiplying both sides of (3-5) and (3-6) by $w_{1}$ and integrating by parts in $Q_{+}$, we have

$$
-\int_{Q_{+}} \sum_{i, j=1,2,3} a_{i j} \partial_{i} w_{1} \partial_{j} w_{1} d X=\sum_{i=1}^{5} J_{i}
$$

where

$$
\begin{aligned}
& J_{1}=\int_{Q_{+}} \sum_{i, j=1,2,3} \partial_{i} a_{i j} \partial_{j} w_{1} w_{1} d X-\int_{Q_{+}} \sum_{i, j=1,2,3} \partial_{1} a_{i j} \partial_{i} w_{j} w_{1} d X \\
& J_{2}=\int_{X_{1}=0} \sum_{j=1,2,3} a_{1 j} \partial_{j} w_{1} w_{1} d S \\
& J_{4}=-\int_{X_{2}^{2}+X_{3}^{2}=1} \sum_{i=2,3}\left(\sum_{j=1,2,3} a_{i j} \partial_{j} w_{1} w_{1}\right) X_{i} d S \\
& J_{3}=-\int_{X_{1}=1} \sum_{j=1,2,3} a_{1 j} \partial_{j} w_{1} w_{1} d S \\
& J_{5}=-\int_{Q_{+}} \partial_{1}\left(\sum_{i=1,2,3} b_{i}(X) \partial_{X_{i}} v+c(X) v\right) w_{1} d X
\end{aligned}
$$

Now we estimate the $J_{i}$ separately.

(i) Estimate of $J_{1}$.

By Lemma 2.4, Sobolev's imbedding theorem, and $\delta_{0}<1 / 2$, we get

$$
\left|J_{1}\right| \leq \delta \varepsilon \int_{Q_{+}} \sum_{j=1,2,3}\left|\nabla w_{j}\right|^{2} d X+C_{\delta} \varepsilon \int_{Q_{+}}\left(\left|w_{1}\right|^{2}+\left|\nabla w_{1}\right|^{2}\right) d X .
$$

Substituting (3-4) into the above expression yields

$$
\left|J_{1}\right| \leq C\left(\delta \varepsilon+C_{\delta} \varepsilon\right) \int_{Q_{+}}\left|\nabla^{2} v\right|^{2} d X .
$$

(ii) Estimate of $J_{2}$ and $J_{3}$. 
Decompose $J_{2}$ as $J_{2}=J_{21}+J_{22}$ with

$$
J_{21}=\int_{X_{1}=0} \sum_{j=2,3} a_{1 j} \partial_{j} w_{1} w_{1} d S \quad \text { and } \quad J_{22}=\int_{X_{1}=0} a_{11} \partial_{1} w_{1} w_{1} d S .
$$

We treat the term $J_{21}$ first. Integrating by parts leads to

$$
2 J_{21}=-\int_{X_{1}=0} \sum_{j=2,3} \partial_{j} a_{1 j} w_{1}^{2} d S+\int_{L} \sum_{j=2,3} a_{1 j} X_{j} w_{1}^{2} d l,
$$

where $L=\left\{X: X_{1}=0, X_{2}^{2}+X_{3}^{2}=1\right\}$.

The first term on the right hand side above can be treated by Lemma 2.4 and Sobolev's imbedding theorem, giving

$$
\left|\int_{X_{1}=0} \sum_{j=2,3} \partial_{j} a_{1 j} w_{1}^{2} d S\right| \leq C \int_{X_{1}=0} \frac{\varepsilon w_{1}^{2}}{R^{\delta_{0}}} d S \leq C \varepsilon \int_{Q_{+}}\left(\left|w_{1}\right|^{2}+\left|\nabla w_{1}\right|^{2}\right) d X .
$$

It is more difficult to treat the second term in $2 J_{21}$ because one cannot use the trace theorem to directly control $\int_{L}\left|w_{1}\right|^{2} d l$ by $\int_{Q_{+}}\left(\left|w_{1}\right|^{2}+\left|\nabla w_{1}\right|^{2}\right) d X$. To overcome this difficulty, we will use $\int_{L}\left|\partial_{\theta} v\right|^{2} d l$ to control $\int_{L}\left|w_{1}\right|^{2} d l$, since $\int_{L}\left|\partial_{\theta} v\right|^{2} d l$ can be estimated by the trace theorem; here $\partial_{\theta}=X_{2} \partial_{3}-X_{3} \partial_{2}$.

Indeed, it follows from the trace theorem that

$$
\int_{L}\left|\partial_{\theta} v\right|^{2} d l \leq\left\|\left.v\right|_{L}\right\|_{H^{1}(L)}^{2} \leq C \int_{Q_{+}}\left(|v|^{2}+|\nabla v|^{2}+\left|\nabla^{2} v\right|^{2}\right) d X .
$$

Additionally, by the boundary conditions in (3-1) and the expression of $\partial_{\theta} v$, we find that, on $L$,

$$
\begin{aligned}
w_{1}+\sum_{i=2,3} \gamma_{1 i} w_{i}+d_{1} v & =0, \\
\sum_{i=1,2,3} \gamma_{3 i} w_{i}+d_{3} v & =0, \\
X_{2} w_{3}-X_{3} w_{2} & =\partial_{\theta} v .
\end{aligned}
$$

By Lemma 3.1, we obtain from (3-9) that on the curve $L$

$$
w_{i}=C_{i 1}(X) d_{1} v+C_{i 2}(X) d_{3} v+C_{i 3}(X) \partial_{\theta} v \quad \text { for } i=1,2,3,
$$

where $\left|C_{i j}(X)\right| \leq C$.

It follows from (3-10), (3-8), Lemma 3.2, and (3-4) that

$$
\begin{aligned}
\left|\int_{L} \sum_{j=2,3} a_{1 j} X_{j} w_{1}^{2} d l\right| & \leq C \varepsilon \int_{L}\left|w_{1}\right|^{2} d l \\
& \leq C \varepsilon \int_{L}\left(\left|\partial_{\theta} v\right|^{2}+|v|^{2}\right) d l \leq C \varepsilon \int_{Q_{+}}\left|\nabla^{2} v\right|^{2} d X .
\end{aligned}
$$


Hence

$$
\left|J_{21}\right| \leq C \varepsilon \int_{Q_{+}}\left|\nabla^{2} v\right|^{2} d X .
$$

We now estimate the term $J_{22}$. From (3-1), we derive that

$$
\partial_{1} w_{1}=-\frac{1}{a_{11}}\left(\sum_{j=2,3} a_{1 j} \partial_{j} w_{1}+\sum_{i, j=2,3} a_{i j} \partial_{i} w_{j}+\sum_{i=1,2,3} b_{i}(X) \partial_{X_{i}} v+c(X) v\right) .
$$

Then

$$
\left|J_{22}\right| \leq\left|J_{21}\right|+\left|J_{22}^{\prime}\right|+\left|\int_{X_{1}=0}\left(\sum_{i=1,2,3} b_{i}(X) \partial_{X_{i}} v+c(X) v\right) w_{1} d S\right|
$$

with $J_{22}^{\prime}=-\int_{X_{1}=0} \sum_{i, j=2,3} a_{i j} \partial_{i} w_{j} w_{1} d S$.

Substituting the boundary condition in (3-1) into $J_{22}^{\prime}$ yields

$$
J_{22}^{\prime}=\int_{X_{1}=0} \sum_{i, j=2,3} a_{i j} \partial_{i} w_{j}\left(\sum_{k=2,3} \gamma_{1 k} w_{k}+d_{1} v\right) d S .
$$

Since $\partial_{i} w_{j} w_{k}=\frac{1}{2}\left(\partial_{i}\left(w_{j} w_{k}\right)-\partial_{k}\left(w_{i} w_{j}\right)+\partial_{j}\left(w_{i} w_{k}\right)\right)$, treatment analogous to that of $J_{21}$ gives $\left|J_{22}^{\prime}\right| \leq C \varepsilon \int_{Q_{+}}\left|\nabla^{2} v\right|^{2} d X$. Thus

$$
\left|J_{2}\right| \leq C \varepsilon \int_{Q_{+}}\left|\nabla^{2} v\right|^{2} d X
$$

(iii) Estimate of $J_{4}$.

Set $J_{4}=J_{41}+J_{42}$ with

$$
J_{41}=-\int_{X_{2}^{2}+X_{3}^{2}=1} \sum_{i=2,3} X_{i} a_{i 1} \partial_{1} w_{1} w_{1} d S \quad \text { and } \quad J_{42}=-\int_{X_{2}^{2}+X_{3}^{2}=1} \sum_{i, j=2,3} X_{i} a_{i j} \partial_{j} w_{1} w_{1} d S .
$$

Since

$$
J_{41}=-\frac{1}{2} \int_{X_{2}^{2}+X_{3}^{2}=1} \partial_{1}\left(\sum_{i=2,3} X_{i} a_{i 1} w_{1}^{2}\right) d S+\frac{1}{2} \int_{X_{2}^{2}+X_{3}^{2}=1} \sum_{i=2,3} \partial_{1}\left(X_{i} a_{i 1}\right) w_{1}^{2} d S,
$$

we have

$$
\left|J_{41}\right| \leq C \varepsilon \int_{L}\left|w_{1}\right|^{2} d l+C \varepsilon\left(\int_{X_{2}^{2}+X_{3}^{2}=1}\left|w_{1}\right|^{4} d S\right)^{1 / 2} \leq C \varepsilon \int_{Q_{+}}\left|\nabla^{2} v\right|^{2} d X .
$$

Next we estimate $J_{42}$. In terms of the cylindrical coordinates

$$
X_{1}=X_{1}, \quad X_{2}=r \cos \theta, \quad X_{3}=r \sin \theta,
$$


we have

$$
\begin{array}{r}
J_{42}=-\int_{0}^{1} \int_{0}^{2 \pi}\left(\left(a_{22} \cos ^{2} \theta+a_{33} \sin ^{2} \theta+a_{23} \sin 2 \theta\right) \partial_{r} w_{1} w_{1}\right. \\
\left.\quad+\left(a_{23} \cos 2 \theta+\left(a_{33}-a_{22}\right) \sin \theta \cos \theta\right) \partial_{\theta} w_{1} w_{1}\right) d \theta d X_{1} .
\end{array}
$$

It follows from the third boundary condition in (3-6) that, on $X_{2}^{2}+X_{3}^{2}=1$,

$$
\partial_{r} w_{1}=D_{11}(X) \partial_{1} w_{1}+D_{12}(X) \partial_{\theta} w_{1}+\sum_{i=1,2,3} D_{13}^{i}(X) w_{i}+D_{14}(X) v
$$

where $\left|D_{11}(X)\right|+\left|D_{12}(X)\right| \leq C \varepsilon$,

$$
\left|\nabla D_{11}(X)\right|+\left|\nabla D_{12}(X)\right| \leq \frac{C \varepsilon}{R^{\delta_{0}}}, \quad \text { and } \quad \sum_{i=1,2,3}\left|D_{13}^{i}(X)\right|+\left|D_{14}(X)\right| \leq \frac{C \varepsilon}{R^{\delta_{0}}}
$$

By Lemma 2.4, integration by parts, and (3-8), we get

$$
\begin{aligned}
\left|J_{42}\right| & \leq C \varepsilon\left(\int_{Q_{+}}\left(\left|w_{1}\right|^{2}+\left|\nabla w_{1}\right|^{2}\right) d X+\int_{L}\left|\partial_{\theta} v\right|^{2} d l+\int_{L} \frac{|v|^{2}}{R^{2 \delta_{0}}} d l\right) \\
& \leq C \varepsilon \int_{Q_{+}}\left|\nabla^{2} v\right|^{2} d X .
\end{aligned}
$$

Hence we have $\left|J_{4}\right| \leq C \varepsilon \int_{Q_{+}}\left|\nabla^{2} v\right|^{2} d X$.

(iv) Estimate of $\mathrm{J}_{5}$.

$$
\left|J_{5}\right| \leq C \varepsilon\left(\int_{Q_{+}} \frac{|\nabla v|^{2}+|v|^{2}}{R^{1+\delta_{0}}} d X+\int_{Q_{+}} \frac{\left|\nabla^{2} v\right||\nabla v|}{R^{\delta_{0}}} d X\right)
$$

Since $H^{1}\left(\bar{Q}_{+}\right) \subset L^{6}\left(Q_{+}\right)$and $0<\delta_{0}<1 / 3$, we have

$$
\begin{aligned}
\int_{Q_{+}} \frac{|\nabla v|^{2}}{R^{1+\delta_{0}}} d X & \leq\left(\int_{Q_{+}} \frac{d X}{R^{3\left(1+\delta_{0}\right) / 2}}\right)^{2 / 3}\left(\int_{Q_{+}}|\nabla v|^{6} d X\right)^{1 / 3} \\
& \leq C \int_{Q_{+}}\left(|\nabla v|^{2}+\left|\nabla^{2} v\right|^{2}\right) d X, \\
\int_{Q_{+}} \frac{|v|^{2}}{R^{1+\delta_{0}}} d X & \leq C\left(\int_{Q_{+}}|v|^{6} d X\right)^{1 / 3} \leq C \int_{Q_{+}}\left(|v|^{2}+|\nabla v|^{2}\right) d X, \\
\int_{Q_{+}} \frac{\left|\nabla^{2} v\right||\nabla v|}{R^{\delta_{0}}} d X & \leq \int_{Q_{+}}\left|\nabla^{2} v\right|^{2} d X+\int_{Q_{+}} \frac{|\nabla v|^{2}}{R^{2 \delta_{0}}} d X \\
& \leq C \int_{Q_{+}}\left(|\nabla v|^{2}+\left|\nabla^{2} v\right|^{2}\right) d X .
\end{aligned}
$$

It follows that $\left|J_{5}\right| \leq C \varepsilon \int_{Q_{+}}\left|\nabla^{2} v\right|^{2} d X$. 
Finally, substituting the estimates of $J_{i}$ for $i=1, \ldots, 5$ into (3-7) yields

$$
\int_{Q_{+}}\left|\nabla \partial_{1} v\right|^{2} d X \leq C \varepsilon \int_{Q_{+}}\left|\nabla^{2} v\right|^{2} d X
$$

Step 3: Estimate of $\sum_{\boldsymbol{k}=\mathbf{2 , 3}}\left\|\nabla \partial_{\boldsymbol{k}} \boldsymbol{v}\right\|_{\boldsymbol{L}^{2}\left(Q_{+}\right)}$. Since $w_{k}=\partial_{k} v$ for $k=2,3$ satisfies

$$
\sum_{i, j=1,2,3} a_{i j} \partial_{i j}^{2} w_{k}+\sum_{i, j=1,2,3} \partial_{k} a_{i j} \partial_{i} w_{j}+\partial_{k}\left(\sum_{i=1,2,3} b_{i}(X) \partial_{X_{i}} v+c(X) v\right)=0
$$

and

$$
\begin{aligned}
& \partial_{1} w_{k}+\sum_{i=2,3} \gamma_{1 i} \partial_{i} w_{k}+\sum_{i=2,3} \partial_{k} \gamma_{1 i} w_{i}+\partial_{k}\left(d_{1} v\right)=0 \quad \text { on } X_{1}=0 \text {, } \\
& \partial_{1} w_{k}+\sum_{i=2,3} \gamma_{2 i} \partial_{i} w_{k}+\sum_{i=2,3} \partial_{k} \gamma_{2 i} w_{i}+\partial_{k}\left(d_{2} v\right)=0 \quad \text { on } X_{1}=1 \text {, } \\
& \sum_{i=1,2,3} \gamma_{3 i} w_{i}+d_{3} v=0 \quad \text { on } X_{2}^{2}+X_{3}^{2}=1 \text {, }
\end{aligned}
$$

multiplying these equations by $w_{k}$ and integrating by parts in $Q_{+}$gives

$$
-\int_{Q_{+}} \sum_{k=2,3} \sum_{i, j=1,2,3} a_{i j} \partial_{i} w_{k} \partial_{j} w_{k} d X=\sum_{i=1}^{5} K_{i},
$$

where

$$
\begin{aligned}
K_{1} & =\int_{Q_{+}} \sum_{k=2,3} \sum_{i, j=1,2,3} \partial_{i} a_{i j} \partial_{j} w_{k} w_{k} d X-\int_{Q_{+}} \sum_{k=2,3} \sum_{i, j=1,2,3} \partial_{k} a_{i j} \partial_{i} w_{j} w_{k} d X, \\
K_{2} & =\int_{X_{1}=0} \sum_{k=2,3} \sum_{j=1,2,3} a_{1 j} \partial_{j} w_{k} w_{k} d S, \\
K_{3}= & -\int_{X_{1}=1} \sum_{k=2,3} \sum_{j=1,2,3} a_{1 j} \partial_{j} w_{k} w_{k} d S, \\
K_{4}= & -\int_{X_{2}^{2}+X_{3}^{2}=1} \sum_{k=2,3} \sum_{i=2,3}\left(\sum_{j=1,2,3} a_{i j} \partial_{j} w_{k} w_{k}\right) X_{i} d S, \\
K_{5}= & -\int_{Q_{+}} \sum_{k=2,3} \partial_{k}\left(\sum_{i=1,2,3} b_{i}(X) \partial_{X_{i}} v+c(X) v\right) w_{k} d X .
\end{aligned}
$$

We can treat the terms $K_{i}$ for $i=1,2,3,5$ like we treated the $J_{i}$ for $i=1,2,3,5$ in Step 2. That is, we have

$$
\left|K_{1}\right|+\left|K_{2}\right|+\left|K_{3}\right|+\left|K_{5}\right| \leq C \varepsilon \int_{Q_{+}}\left|\nabla^{2} v\right|^{2} d X
$$


However, it seems difficult to control $\left|K_{4}\right|$ in terms of $C \varepsilon \int_{Q_{+}}\left|\nabla^{2} v\right|^{2} d X$ since it contains a term

$$
-\int_{X_{2}^{2}+X_{3}^{2}=1}\left|\partial_{\theta} v\right|^{2} d S
$$

with no small coefficients. So additional care is needed. Our main observation is that

$$
K_{4}-\int_{X_{2}^{2}+X_{3}^{2}=1} a_{22}\left|\partial_{\theta} v\right|^{2} d S \geq-C \varepsilon \int_{Q_{+}}\left|\nabla^{2} v\right|^{2} d X,
$$

which, together with (3-13) and (3-14), yields the derived estimate.

We now verify (3-15). First we decompose $K_{4}$ as $K_{4}=K_{41}+K_{42}$ with

$$
\begin{aligned}
K_{41} & =-\int_{X_{2}^{2}+X_{3}^{2}=1} \sum_{k=2,3}\left(\sum_{i=2,3} a_{i 1} \partial_{1} w_{k} w_{k} X_{i}\right) d S, \\
K_{42} & =-\int_{X_{2}^{2}+X_{3}^{2}=1} \sum_{k=2,3} \sum_{i, j=2,3} a_{i j} X_{i} \partial_{j} w_{k} w_{k} d S .
\end{aligned}
$$

In a similar way as for $J_{41}$, we can show

$$
\left|K_{41}\right| \leq C \varepsilon \int_{L}\left|w_{k}\right|^{2} d l+C \varepsilon\left(\int_{X_{2}^{2}+X_{3}^{2}=1}\left|w_{k}\right|^{4} d S\right)^{1 / 2} \leq C \varepsilon \int_{Q_{+}}\left|\nabla^{2} v\right|^{2} d X .
$$

It remains to estimate $K_{42}$. Using the cylindrical coordinate transformation (3-11), we have $K_{42}=K_{42}^{\prime}+K_{42}^{\prime \prime}$ with

$$
\begin{array}{r}
K_{42}^{\prime}=-\int_{X_{2}^{2}+X_{3}^{2}=1} a_{22}\left(\partial_{r}^{2} v \partial_{r} v+\partial_{r \theta}^{2} v \partial_{\theta} v-\left(\partial_{\theta} v\right)^{2}\right) d S, \\
K_{42}^{\prime \prime}=\int_{X_{2}^{2}+X_{3}^{2}=1}\left(E_{11}(X) \partial_{r}^{2} v+E_{12}(X) \partial_{r \theta}^{2} v+E_{13}(X) \partial_{\theta}^{2} v+E_{14}(X) \partial_{r} v+E_{15}(X) \partial_{\theta} v\right) \\
\times\left(H_{1}(\theta) \partial_{r} v+H_{2}(\theta) \partial_{\theta} v\right) d S,
\end{array}
$$

where $\sum_{i=1}^{5}\left|E_{1 i(X)}\right| \leq C \varepsilon, \sum_{i=1}^{5}\left|\nabla E_{1 i}(X)\right| \leq C \varepsilon / R^{\delta_{0}}$, and $H_{i}(\theta)$ for $i=1,2$ are smooth functions on $\theta$.

From (3-1), we have on $X_{2}^{2}+X_{3}^{2}=1$

$$
\begin{aligned}
\partial_{r}^{2} v=E_{21}(X) \partial_{1}^{2} v+ & E_{22} \partial_{\theta}^{2} v+E_{23}(X) \partial_{1 r}^{2} v+E_{24}(X) \partial_{1 \theta}^{2} v \\
& +E_{25}(X) \partial_{r \theta}^{2} v+E_{26}(X) \partial_{\theta} v+E_{27} \partial_{r} v+E_{28}(X) v
\end{aligned}
$$

where $\left|E_{21}\right|+\left|E_{22}\right| \leq C$,

$$
\sum_{j=1}^{5}\left|\nabla E_{2 j}\right|+\sum_{j=6}^{8}\left|E_{2 j}\right| \leq \frac{C \varepsilon}{R^{\delta_{0}}}, \quad \sum_{j=3}^{5}\left|E_{2 j}\right|+\left|E_{28}\right| \leq C \varepsilon, \quad \sum_{j=6}^{8}\left|\nabla E_{2 j}\right| \leq \frac{C \varepsilon}{R^{1+\delta_{0}}} .
$$


Additionally, it follows from the boundary conditions in (3-1) that

$$
\partial_{r} v=E_{31}(X) \partial_{1} v+E_{32}(X) \partial_{\theta} v+E_{33}(X) v \quad \text { on } X_{2}^{2}+X_{3}^{2}=1,
$$

where $\sum_{j=1,2,3}\left|E_{3 j}\right| \leq C \varepsilon$ and $\sum_{j=1,2,3}\left|\nabla E_{3 j}\right| \leq C \varepsilon / R^{\delta_{0}}$.

Substituting (3-16) and (3-17) into $K_{42}^{\prime}$ yields

$$
K_{42}^{\prime}=\int_{X_{2}^{2}+X_{3}^{2}=1} a_{22}\left(\partial_{\theta} v\right)^{2} d S+\left|\int_{X_{2}^{2}+X_{3}^{2}=1} G\left(\partial_{1} v, \partial_{1}^{2} v, \partial_{\theta} v, \partial_{\theta}^{2} v, \partial_{1 \theta}^{2} v\right) d S\right|
$$

with

$G\left(\partial_{1} v, \partial_{1}^{2} v, \partial_{\theta} v, \partial_{\theta}^{2} v, \partial_{1 \theta}^{2} v\right)=E_{51}(X) \partial_{1}^{2} v \partial_{1} v+E_{52}(X) \partial_{1}^{2} v \partial_{\theta} v+E_{53}(X) \partial_{\theta}^{2} v \partial_{1} v$

$$
+E_{54}(X) \partial_{\theta}^{2} v \partial_{\theta} v+E_{55}(X) \partial_{1 \theta}^{2} v \partial_{1} v
$$

$+E_{56}(X) \partial_{1 \theta}^{2} v \partial_{\theta} v+E_{57}(X)\left(\partial_{1} v\right)^{2}+E_{58}(X) \partial_{1} v \partial_{\theta} v+E_{59}(X)\left(\partial_{\theta} v\right)^{2}$

$+N_{1}(X) \partial_{1}^{2} v v+N_{2}(X) \partial_{\theta}^{2} v v+N_{3}(X) \partial_{1} v v+N_{4}(X) \partial_{\theta} v v+N_{5}(X) v^{2}$,

where

$$
\begin{array}{rlrl}
\sum_{i=1}^{6}\left|\nabla E_{5 i}(X)\right|+ & \sum_{i=7}^{9}\left|E_{5 i}(X)\right| \leq \frac{C \varepsilon}{R^{\delta_{0}}}, & \sum_{j=1}^{2}\left|\nabla N_{j}(X)\right|+\sum_{j=3}^{5}\left|N_{j}(X)\right| \leq \frac{C \varepsilon}{R^{\delta_{0}}} \\
\sum_{i=1}^{6}\left|E_{5 i}(X)\right| \leq C \varepsilon, & \sum_{j=1}^{2}\left|N_{j}(X)\right| \leq C \varepsilon, \\
\sum_{i=7}^{9}\left|\nabla E_{5 i}(X)\right| \leq \frac{C \varepsilon}{R^{1+\delta_{0}}}, & \sum_{j=3}^{5}\left|\nabla N_{j}(X)\right| \leq \frac{C \varepsilon}{R^{1+\delta_{0}}} .
\end{array}
$$

Similar to the treatment of $J_{42}$ in Step 2, we have

$$
\begin{aligned}
\left|\int_{X_{2}^{2}+X_{3}^{2}=1} G d S\right| & \leq C \varepsilon\left(\int_{X_{2}^{2}+X_{3}^{2}=1} \frac{|\nabla v|^{2}+|v|^{2}}{R^{\delta_{0}}} d S+\int_{L}\left(|\nabla v|^{2}+\frac{|v|^{2}}{R^{2 \delta_{0}}}\right) d l\right) \\
& \leq C \varepsilon \int_{Q_{+}}\left|\nabla^{2} v\right|^{2} d X .
\end{aligned}
$$

By the same method, we can conclude that $\left|K_{42}^{\prime \prime}\right| \leq C \varepsilon \int_{Q_{+}}\left|\nabla^{2} v\right|^{2} d X$. Thus it follows from (3-13)-(3-15) that

$$
\int_{Q_{+}} \sum_{k=2,3}\left|\nabla w_{k}\right|^{2} d X+\int_{X_{2}^{2}+X_{3}^{2}=1}\left|\partial_{\theta} v\right|^{2} d S \leq C \varepsilon \int_{Q_{+}}\left|\nabla^{2} v\right|^{2} d X .
$$

Adding (3-12) and (3-18), we obtain

$$
\int_{Q_{+}}\left|\nabla^{2} v\right|^{2} d X+\int_{X_{2}^{2}+X_{3}^{2}=1}\left|\partial_{\theta} v\right|^{2} d S \leq C \varepsilon \int_{Q_{+}}\left|\nabla^{2} v\right|^{2} d X .
$$


Hence if we choose $\varepsilon$ such that $C \varepsilon<1 / 2$, then $\int_{Q_{+}}\left|\nabla^{2} v\right|^{2} d X=0$. In light of Lemma 3.2, we obtain

$$
\int_{Q_{+}}\left(|v|^{2}+|\nabla v|^{2}+\left|\nabla^{2} v\right|^{2}\right) d X=0 .
$$

Thus $v \equiv 0$, and the proof of Lemma 3.3 is completed.

Proof of Theorem 1.1. Based on Lemma 3.3, it follows from the transformation (2-3) and the definition of $V(X)$ in (2-4) that the solution $\left(\varphi_{+}(x), \xi\left(x_{2}, x_{3}\right)\right)$ in Theorem 1.1 is unique.

\section{On the nonexistence}

Here, we study the potential equation and the nonexistence of its transonic shock wave patterns for a class of nozzles. In particular, we will prove Theorem 1.5.

It is more convenient to analyze the transonic shock problem in the class of nozzles given in the Theorem 1.5 in the spherical coordinates

$$
x_{1}=x_{1}^{0}+r \cos \alpha, \quad x_{2}=r \sin \alpha \cos \theta, \quad x_{3}=r \sin \alpha \sin \theta
$$

with $0 \leq \alpha \leq \alpha_{0}$ and $0 \leq \theta<2 \pi$.

In these coordinates, Equation (1-3) for $\varphi_{+}$can be written as

$$
\begin{aligned}
c^{2}\left(H_{+}\right)\left(\frac{1}{r^{2}} \partial_{r}\left(r^{2} \partial_{r} \varphi_{+}\right)+\frac{1}{r^{2} \sin \alpha} \partial_{\alpha}\left(\sin \alpha \partial_{\alpha} \varphi_{+}\right)+\frac{1}{r^{2} \sin ^{2} \alpha} \partial_{\theta}^{2} \varphi_{+}\right) \\
-\frac{1}{2}\left(\partial_{r} \varphi_{+} \partial_{r}+\frac{1}{r^{2} \sin ^{2} \alpha} \partial_{\theta} \varphi_{+} \partial_{\theta}+\frac{1}{r^{2}} \partial_{\alpha} \varphi_{+} \partial_{\alpha}\right)\left(\left|\nabla \varphi_{+}\right|^{2}\right)=0,
\end{aligned}
$$

where

$$
H_{+}=H\left(C_{0}-\frac{1}{2}\left(\left(\partial_{r} \varphi_{+}\right)^{2}+\frac{1}{r^{2} \sin ^{2} \alpha}\left(\partial_{\theta} \varphi_{+}\right)^{2}+\frac{1}{r^{2}}\left(\partial_{\alpha} \varphi_{+}\right)^{2}\right)\right) .
$$

Suppose that the shock surface $\Sigma: x_{1}=\xi\left(x_{2}, x_{3}\right)$ is given by $r=r(\theta, \alpha)-x_{1}^{0}$ with $0 \leq \theta<2 \pi$ and $0 \leq \alpha \leq \alpha_{0}$, and the fixed point $\tilde{x}^{0}$ is expressed by

$$
\tilde{x}^{0}=\left(x_{1}^{0}+r^{0} \cos \alpha_{0}, r^{0} \sin \alpha_{0} \cos \tilde{\theta}_{0}, r^{0} \sin \alpha_{0} \sin \tilde{\theta}_{0}\right)
$$

with $r^{0}=\left(\left(x_{1}^{0}-\tilde{x}_{1}^{0}\right)^{2}+\left(\tilde{x}_{2}^{0}\right)^{2}+\left(\tilde{x}_{3}^{0}\right)^{2}\right)^{1 / 2}$. Since $\Sigma$ goes through the point $\tilde{x}^{0}$ and $r^{0}=-x_{1}^{0}$, we have

$$
r\left(\tilde{\theta}_{0}, \alpha_{0}\right)=0 .
$$

In addition, the corresponding boundary conditions (1-9), (1-10), (1-12), and (1-13) become

$$
\begin{aligned}
& \varphi_{+}=\varphi_{-} \quad \text { on } \Sigma, \\
& \partial_{\alpha} \varphi_{+}=0 \quad \text { on } \Pi_{2} \text {. }
\end{aligned}
$$


On $\Sigma$, they become

$$
\begin{aligned}
\left(\left(\partial_{r} \varphi_{+} \partial_{r}\right.\right. & \left.\left.+\frac{1}{r^{2} \sin ^{2} \alpha} \partial_{\theta} \varphi_{+} \partial_{\theta}+\frac{1}{r^{2}} \partial_{\alpha} \varphi_{+} \partial_{\alpha}\right)\left(\varphi_{+}-\varphi_{-}\right)\right) H_{+} \\
& -\left(\left(\partial_{r} \varphi_{-} \partial_{r}+\frac{1}{r^{2} \sin ^{2} \alpha} \partial_{\theta} \varphi_{-} \partial_{\theta}+\frac{1}{r^{2}} \partial_{\alpha} \varphi_{-} \partial_{\alpha}\right)\left(\varphi_{+}-\varphi_{-}\right)\right) H_{-}=0
\end{aligned}
$$

Finally, on $r=r_{1}=\left(1-x_{1}^{0}\right) \sec \alpha_{0}$, they become

$$
H\left(C_{0}-\frac{1}{2}\left(\left(\partial_{r} \varphi_{+}\right)^{2}+\frac{1}{r^{2} \sin ^{2} \alpha}\left(\partial_{\theta} \varphi_{+}\right)^{2}+\frac{1}{r^{2}}\left(\partial_{\alpha} \varphi_{+}\right)^{2}\right)\right)=\rho_{+},
$$

It follows from Theorem 1.1 and Remark 1.3 that the solution in Theorem 1.5 is unique if it exists and satisfies the regularity assumptions in Theorem 1.1. However, by the symmetric properties of the widening part of the nozzle and the supersonic incoming flow, one can improve the regularities of $\varphi_{+}$in $\bar{\Omega}_{+}$as follows:

Proposition 4.1. Under the regularity assumptions in Theorem 1.1 and (1-15)', for any fixed constant $\delta_{0}^{\prime}$ with $\delta_{0}<\delta_{0}^{\prime}<1$, the solution pair $\left(\varphi_{+}(x), \xi\left(x_{2}, x_{3}\right)\right)$ to the problem (1-3) with (1-8)-(1-11), (1-12)', and (1-13) has the following higher regularities in $\bar{\Omega}_{+}$:

(i) $\xi\left(x_{2}, x_{3}\right) \in C^{2,1-\delta_{0}^{\prime}}(\bar{S}) \cap C^{3}(S)$ and $\left\|\xi\left(x_{2}, x_{3}\right)\right\|_{C^{2,1-\delta_{0}^{\prime}}(\bar{S})} \leq C \varepsilon$.

(ii) $\varphi_{+}(x) \in C^{2,1-\delta_{0}^{\prime}}\left(\bar{\Omega}_{+}\right)$such that $\left\|\varphi_{+}(x)-q_{+} x_{1}\right\|_{C^{2,1-\delta_{0}^{\prime}\left(\bar{\Omega}_{+}\right)}} \leq C \varepsilon$.

Remark 4.2. In fact, we can show that $\xi\left(x_{2}, x_{3}\right) \in C^{2,1-\delta_{0}}(\bar{S}) \cap C^{3}(S)$ and $\varphi_{+}(x) \in$ $C^{2,1-\delta_{0}}\left(\bar{\Omega}_{+}\right)$. But the regularities in Proposition 4.1 are enough for the proof of Theorem 1.5.

Proof. It suffices to show $\varphi_{+}(x) \in C^{2,1-\delta_{0}^{\prime}}\left(\bar{\Omega}_{+}\right)$.

To this end, as in Section 2, we will use a partial hodograph transformation to rewrite the problem (4-2)-(4-6) as a fixed boundary value problem.

Set

$$
\begin{aligned}
& \tilde{r}=-x_{1}^{0}+\left(1-\frac{r_{1}-r}{r_{1}-r+\varphi_{-}(r)-\varphi_{+}(r, \theta, \alpha)}\right)\left(r_{1}+x_{1}^{0}\right), \\
& \tilde{\theta}=\theta, \\
& \tilde{\alpha}=\alpha
\end{aligned}
$$

and $V(r, \theta, \alpha)=r_{1}-r+\varphi_{-}(r)-\varphi_{+}(r, \theta, \alpha)$, where $r_{1}=\left(1-x_{1}^{0}\right) \sec \alpha_{0}$.

In terms of (4-7), the domain $\Omega_{+}$is transformed into the domain

$$
Q_{+}=\left\{(\tilde{r}, \tilde{\theta}, \tilde{\alpha}):-x_{1}^{0}<\tilde{r}<r_{1}, 0 \leq \theta<2 \pi, 0 \leq \alpha<\alpha_{0}\right\} .
$$


Then, a direct computation yields

$$
r=r_{1}-\frac{r_{1}-\tilde{r}}{r_{1}+x_{1}^{0}} V, \quad \partial_{r} \varphi_{+}=-1+\varphi_{-}^{\prime}(r)-\partial_{r} V, \quad \partial_{r}^{2} \varphi_{+}=\varphi_{-}^{\prime \prime}(r)-\partial_{r}^{2} V,
$$

$$
\begin{aligned}
& \partial_{\alpha} \varphi_{+}=-\partial_{\alpha} V, \quad \partial_{r \alpha}^{2} \varphi_{+}=-\partial_{r \alpha}^{2} V, \quad \partial_{\alpha}^{2} \varphi_{+}=-\partial_{\alpha}^{2} V, \\
& \partial_{\theta} \varphi_{+}=-\partial_{\theta} V, \quad \partial_{r \theta}^{2} \varphi_{+}=-\partial_{r \theta}^{2} V, \quad \partial_{\theta}^{2} \varphi_{+}=-\partial_{\theta}^{2} V, \\
& \partial_{r} V=A_{0}\left(r_{1}+x_{1}^{0}\right) \partial_{\tilde{r}} V, \quad \partial_{r}^{2} V=\partial_{\tilde{r}}\left(\partial_{r} V\right) \partial_{r} \tilde{r}, \quad \partial_{r \theta}^{2} V=\partial_{\tilde{r}}\left(\partial_{\theta} V\right) \partial_{r} \tilde{r}, \\
& \partial_{\alpha} V=A_{0} V \partial_{\tilde{\alpha}} V, \quad \partial_{\theta} V=A_{0} V \partial_{\tilde{\theta}} V, \quad \partial_{r \alpha}^{2} V=\partial_{\tilde{r}}\left(\partial_{\alpha} V\right) \partial_{r} \tilde{r}, \\
& \partial_{\alpha}^{2} V=\partial_{\tilde{\alpha}}\left(\partial_{\alpha} V\right)+\partial_{\tilde{r}}\left(\partial_{\alpha} V\right) \partial_{\alpha} \tilde{r} \\
& \partial_{\theta}^{2} V=\partial_{\tilde{\theta}}\left(\partial_{\theta} V\right)+\partial_{\tilde{r}}\left(\partial_{\theta} V\right) \partial_{\theta} \tilde{r},
\end{aligned}
$$

where $A_{0}=1 /\left(V+\left(\tilde{r}-r_{1}\right) \partial_{\tilde{r}} V\right)$. Here

$$
\partial_{r} \tilde{r}=\frac{r_{1}+x_{1}^{0}}{V}+\frac{\left(r_{1}-\tilde{r}\right) \partial_{r} V}{V}, \quad \partial_{\alpha} \tilde{r}=\frac{\left(r_{1}-\tilde{r}\right) \partial_{\alpha} V}{V}, \quad \partial_{\theta} \tilde{r}=\frac{\left(r_{1}-\tilde{r}\right) \partial_{\theta} V}{V} .
$$

Next we study the relations among $\partial_{\tilde{\theta}} V, \partial_{\tilde{r}} V, \partial_{\tilde{\theta}}^{2} V, \partial_{\tilde{r}}^{2} V$ and $\left(X_{2} \partial_{X_{3}}-X_{3} \partial_{X_{2}}\right) W$ in $(1-15)^{\prime}$.

It follows from (4-1), (4-7), and (1-14) that

$$
\begin{aligned}
& X_{1}=1-\left(\left(\left(1-x_{1}^{0}\right)^{2} \sec ^{2} \alpha_{0}-r^{2} \sin ^{2} \tilde{\alpha}\right)^{1 / 2}-r \cos \tilde{\alpha}\right) / W, \\
& X_{2}=\operatorname{ctg} \alpha_{0} \operatorname{tg} \tilde{\alpha} \cos \tilde{\theta}, \\
& X_{3}=\operatorname{ctg} \alpha_{0} \operatorname{tg} \tilde{\alpha} \sin \tilde{\theta},
\end{aligned}
$$

where

$$
\begin{aligned}
W & =\left(\left(1-x_{1}^{0}\right)^{2} \sec ^{2} \alpha_{0}-r^{2} \sin ^{2} \tilde{\alpha}\right)^{1 / 2}-r \cos \tilde{\alpha}+V-r_{1}+r, \\
r & =r_{1}-\left(\left(r_{1}-\tilde{r}\right) /\left(r_{1}+x_{1}^{0}\right)\right) V .
\end{aligned}
$$

Since $\tilde{\theta}=\operatorname{arctg}\left(X_{3} / X_{2}\right)$ and $\tilde{\alpha}=\operatorname{arctg}\left(\operatorname{tg} \alpha_{0}\left(X_{2}^{2}+X_{3}^{2}\right)^{1 / 2}\right.$, we have

$$
\begin{array}{ll}
\partial_{X_{2}} \tilde{\theta}=-\operatorname{tg} \alpha_{0} \sin \tilde{\theta} /(\operatorname{tg} \tilde{\alpha}), & \partial_{X_{3}} \tilde{\theta}=\operatorname{tg} \alpha_{0} \cos \tilde{\theta} /(\operatorname{tg} \tilde{\alpha}), \\
\partial_{X_{2}} \tilde{\alpha}=t g \alpha_{0} \cos \tilde{\theta} /\left(1+\operatorname{tg}^{2} \tilde{\alpha}\right), & \partial_{X_{3}} \tilde{\alpha}=\operatorname{tg} \alpha_{0} \sin \tilde{\theta} /\left(1+t^{2} \tilde{\alpha}\right)
\end{array}
$$

and

$$
\begin{aligned}
& \partial_{X_{2}}=\partial_{X_{2}} \tilde{\theta} \partial_{\tilde{\theta}}+\partial_{X_{2}} \tilde{\alpha} \partial_{\tilde{\alpha}}+\partial_{X_{2}} \tilde{r} \partial_{\tilde{r}}=\operatorname{tg} \alpha_{0}\left(-\frac{\sin \tilde{\theta}}{\operatorname{tg} \tilde{\alpha}} \partial_{\tilde{\theta}}+\frac{\cos \tilde{\theta}}{1+\operatorname{tg}^{2} \tilde{\alpha}} \partial_{\tilde{\alpha}}\right)+\partial_{X_{2}} \tilde{r} \partial_{\tilde{r}}, \\
& \partial_{X_{3}}=\partial_{X_{3}} \tilde{\theta} \partial_{\tilde{\theta}}+\partial_{X_{3}} \tilde{\alpha} \partial_{\tilde{\alpha}}+\partial_{X_{3}} \tilde{r} \partial_{\tilde{r}}=\operatorname{tg} \alpha_{0}\left(\frac{\cos \tilde{\theta}}{\operatorname{tg} \tilde{\alpha}} \partial_{\tilde{\theta}}+\frac{\sin \tilde{\theta}}{1+\operatorname{tg}^{2} \tilde{\alpha}} \partial_{\tilde{\alpha}}\right)+\partial_{X_{3}} \tilde{r} \partial_{\tilde{r}} .
\end{aligned}
$$


Hence

$$
\begin{aligned}
X_{2} \partial_{X_{3}}-X_{3} \partial_{X_{2}} & =\partial_{\tilde{\theta}}+\left(X_{2} \partial_{X_{3}}-X_{3} \partial_{X_{2}}\right) \tilde{r} \partial_{\tilde{r}}, \\
\left(X_{2} \partial_{X_{3}}-X_{3} \partial_{X_{2}}\right) \tilde{\alpha} & =0 .
\end{aligned}
$$

In addition, it follows from the first equality in (4-8) and (1-15)' that

$$
\left(X_{2} \partial_{X_{3}}-X_{3} \partial_{X_{2}}\right) r=p_{1}(\tilde{r}, \tilde{\theta}, \tilde{\alpha})
$$

with

$$
\begin{aligned}
p_{1}(\tilde{r}, \tilde{\theta}, \tilde{\alpha}) & =\frac{g}{W \partial_{r} g}\left(X_{2} \partial_{X_{3}}-X_{3} \partial_{X_{2}}\right) W \in C^{1,1-\delta_{0}}\left(\bar{Q}_{+}\right), \\
g(r, \tilde{\alpha}) & =\left(\left(1-x_{1}^{0}\right)^{2} \sec ^{2} \alpha_{0}-r^{2} \sin ^{2} \tilde{\alpha}\right)^{1 / 2}-r \cos \tilde{\alpha},
\end{aligned}
$$

and $\left\|p_{1}(\tilde{r}, \tilde{\theta}, \tilde{\alpha})\right\|_{C^{1,1-\delta_{0}}\left(\bar{Q}_{+}\right)} \leq C \varepsilon$. Thus, by the relation of $V$ and $W,(4-9),(4-10)$, and (1-15)', we can derive that

$$
\left(X_{2} \partial_{X_{3}}-X_{3} \partial_{X_{2}}\right) V=p_{2}(\tilde{r}, \tilde{\theta}, \tilde{\alpha}) \in C^{1,1-\delta_{0}}\left(\bar{Q}_{+}\right)
$$

with $\left\|p_{2}(\tilde{r}, \tilde{\theta}, \tilde{\alpha})\right\|_{C^{1,1-\delta_{0}}\left(\bar{Q}_{+}\right)} \leq C \varepsilon$.

In terms of $r=r_{1}-\left(\left(r_{1}-\tilde{r}\right) /\left(r_{1}+x_{1}^{0}\right)\right) V$, we have

$$
\left(X_{2} \partial_{X_{3}}-X_{3} \partial_{X_{2}}\right) r=\frac{\left(X_{2} \partial_{X_{3}}-X_{3} \partial_{X_{2}}\right) \tilde{r}}{r_{1}+x_{1}^{0}} V-\frac{r_{1}-\tilde{r}}{r_{1}+x_{1}^{0}}\left(X_{2} \partial_{X_{3}}-X_{3} \partial_{X_{2}}\right) V .
$$

Combining this with (4-10) and (4-11) yields $\left(X_{2} \partial_{X_{3}}-X_{3} \partial_{X_{2}}\right) \tilde{r}=p_{3}(\tilde{r}, \tilde{\theta}, \tilde{\alpha})$, where $p_{3}(\tilde{r}, \tilde{\theta}, \tilde{\alpha}) \in C^{1,1-\delta_{0}}\left(\bar{Q}_{+}\right)$and $\left\|p_{3}(\tilde{r}, \tilde{\theta}, \tilde{\alpha})\right\|_{C^{1,1-\delta_{0}}\left(\bar{Q}_{+}\right)} \leq C \varepsilon$.

Therefore, it follows from (4-9) that

$$
\partial_{\tilde{\theta}} V=p_{2}(\tilde{r}, \tilde{\theta}, \tilde{\alpha})-p_{3}(\tilde{r}, \tilde{\theta}, \tilde{\alpha}) \partial_{\tilde{r}} V
$$

and

$$
\left\{\begin{aligned}
\partial_{\tilde{\theta}}^{2} V= & \partial_{\tilde{\theta}} p_{2}(\tilde{r}, \tilde{\theta}, \tilde{\alpha})-\partial_{\tilde{\theta}} p_{3}(\tilde{r}, \tilde{\theta}, \tilde{\alpha}) \partial_{\tilde{r}} V-\left(p_{3}(\tilde{r}, \tilde{\theta}, \tilde{\alpha})\right. \\
& \left.\times\left(\partial_{\tilde{r}} p_{2}(\tilde{r}, \tilde{\theta}, \tilde{\alpha})-\partial_{\tilde{r}} p_{3}(\tilde{r}, \tilde{\theta}, \tilde{\alpha}) \partial_{\tilde{r}} V-p_{3}(\tilde{r}, \tilde{\theta}, \tilde{\alpha}) \partial_{\tilde{r}}^{2} V\right)\right) \\
= & p_{4}(\tilde{r}, \tilde{\theta}, \tilde{\alpha})+p_{3}^{2}(\tilde{r}, \tilde{\theta}, \tilde{\alpha}) \partial_{\tilde{r}}^{2} V \\
\partial_{\tilde{r} \tilde{\theta}}^{2} V= & p_{5}(\tilde{r}, \tilde{\theta}, \tilde{\alpha})-p_{3}(\tilde{r}, \tilde{\theta}, \tilde{\alpha}) \partial_{\tilde{r}}^{2} V, \\
\partial_{\tilde{\alpha} \tilde{\theta}}^{2} V= & p_{6}(\tilde{r}, \tilde{\theta}, \tilde{\alpha})-p_{3}(\tilde{r}, \tilde{\theta}, \tilde{\alpha}) \partial_{\tilde{r} \tilde{\alpha}}^{2} V,
\end{aligned}\right.
$$

with $p_{i}(\tilde{r}, \tilde{\theta}, \tilde{\alpha}) \in C^{1-\delta_{0}}\left(\bar{Q}_{+}\right)$and $\left\|p_{i}(\tilde{r}, \tilde{\theta}, \tilde{\alpha})\right\|_{C^{1-\delta_{0}}\left(\bar{Q}_{+}\right)} \leq C \varepsilon$ for $i=4,5,6$.

Then, direction computations show that the solution to the problem (4-2)-(4-6) solves the two-dimensional problem

$$
a_{11}(\tilde{r}, \tilde{\theta}, \tilde{\alpha}) \partial_{\tilde{r}}^{2} V+2 a_{12}(\tilde{r}, \tilde{\theta}, \tilde{\alpha}) \partial_{\tilde{r} \tilde{\alpha}}^{2} V+a_{22}(\tilde{r}, \tilde{\theta}, \tilde{\alpha}) \partial_{\tilde{\alpha}}^{2} V=f(\tilde{r}, \tilde{\theta}, \tilde{\alpha}),
$$


with

$$
\begin{aligned}
\partial_{\tilde{\alpha}} V & =0 & & \text { on } \tilde{\alpha}=\alpha_{0}, \\
G_{1}\left(\tilde{r}, \tilde{\theta}, \tilde{\alpha}, V, \partial_{\tilde{r}} V,\left(\partial_{\tilde{\alpha}} V\right)^{2}\right) & =0 & & \text { on } \tilde{r}=-x_{1}^{0}, \\
G_{2}\left(\tilde{r}, \tilde{\theta}, \tilde{\alpha}, V, \partial_{\tilde{r}} V,\left(\partial_{\tilde{\alpha}} V\right)^{2}\right) & =\rho_{+} & & \text {on } \tilde{r}=r_{1},
\end{aligned}
$$

where $a_{i j}(\tilde{r}, \tilde{\theta}, \tilde{\alpha})$ and $f(\tilde{r}, \tilde{\theta}, \tilde{\alpha})$ are given explicitly by (A.6) and (A.7) respectively, and $G_{1}$ and $G_{2}$ are derived from the nonlinear boundary conditions in (4-4)(4-6) by expressing $\nabla_{r, \theta, \alpha} \varphi_{+}$in terms of the transformation (4-7) and then using (4-12).

By the regularity assumptions in Theorem 1.5 and (4-13), one can check that, near the curves $\tilde{l}_{1}=\Sigma \cap\left\{\alpha=\alpha_{0}\right\}$ and $\tilde{l}_{2}=\left\{\left(r_{1}, \tilde{\theta}, \alpha_{0}\right): 0 \leq \tilde{\theta}<2 \pi\right\}$, we have $a_{i j}(\tilde{r}, \tilde{\theta}, \tilde{\alpha}) \in C^{1-\delta_{0}}$ and $f(\tilde{r}, \tilde{\theta}, \tilde{\alpha}) \in C^{1-\delta_{0}}$. Also, the second order equation in (4-14) and (4-15) is uniformly elliptic, and $G_{i}\left(\tilde{r}, \tilde{\theta}, \tilde{\alpha}, V, \partial_{\tilde{r}} V,\left(\partial_{\tilde{\alpha}} V\right)^{2}\right)$ with $i=1$ is $C^{1,1-\delta_{0}}$-regular with respect to $(\tilde{r}, \tilde{\theta}, \tilde{\alpha})$; for $i=2$, it is $C^{4}$-regular with respect to $\left(V, \partial_{\tilde{r}} V,\left(\partial_{\tilde{\alpha}} V\right)^{2}\right)$. Furthermore, it follows from $\partial_{\alpha} \varphi_{+}=\partial_{\alpha} V=\partial_{\tilde{\alpha}} V=\partial_{\theta} \varphi_{+}=0$ on $\alpha=\tilde{\alpha}=\alpha_{0}$ that $a_{12}\left(\tilde{r}, \tilde{\theta}, \alpha_{0}\right) \equiv 0$ and $\partial_{\tilde{\alpha}} G_{i}\left(\tilde{r}, \tilde{\theta}, \alpha_{0}, V, \partial_{\tilde{r}} V,\left(\partial_{\tilde{\alpha}} V\right)^{2}\right) \equiv 0$ on $\tilde{\alpha}=\alpha_{0}$. (For the proof that $\partial_{\theta} \varphi_{+} \equiv 0$ on $\tilde{l}_{1}$, see Proposition 4.3 below.)

Next, we use a reflection technique to improve the regularity of $V$ in $\bar{Q}_{+}$.

Without loss of generality, we consider the solution to (4-14) and (4-15) in the domain $D \equiv\left[-x_{1}^{0},-x_{1}^{0}+\delta ; \theta_{0}-\delta, \theta_{0}+\delta ; \alpha_{0}-\delta, \alpha_{0}+\delta\right]$ (that is, a small neighborhood near the intersection curve $\tilde{l}_{1}$ ) with a small constant $\delta>0$. To this end, we need to extend those quantities involved in this domain.

Thus, set

$$
\begin{aligned}
& \bar{V}(\tilde{r}, \tilde{\theta}, \tilde{\alpha})= \begin{cases}V(\tilde{r}, \tilde{\theta}, \tilde{\alpha}) & \text { if } \alpha_{0}-\delta \leq \tilde{\alpha} \leq \alpha_{0}, \\
V\left(\tilde{r}, \tilde{\theta}, 2 \alpha_{0}-\tilde{\alpha}\right) & \text { if } \alpha_{0} \leq \tilde{\alpha} \leq \alpha_{0}+\delta,\end{cases} \\
& \bar{a}_{i i}(\tilde{r}, \tilde{\theta}, \tilde{\alpha})= \begin{cases}a_{i i}(\tilde{r}, \tilde{\theta}, \tilde{\alpha}), & \text { if } \alpha_{0}-\delta \leq \tilde{\alpha} \leq \alpha_{0}, \\
a_{i i}\left(\tilde{r}, \tilde{\theta}, 2 \alpha_{0}-\tilde{\alpha}\right) & \text { if } \alpha_{0} \leq \tilde{\alpha} \leq \alpha_{0}+\delta,\end{cases} \\
& \bar{a}_{12}(\tilde{r}, \tilde{\theta}, \tilde{\alpha})= \begin{cases}a_{12}(\tilde{r}, \tilde{\theta}, \tilde{\alpha}), & \text { if } \alpha_{0}-\delta \leq \tilde{\alpha} \leq \alpha_{0}, \\
-a_{12}\left(\tilde{r}, \tilde{\theta}, 2 \alpha_{0}-\tilde{\alpha}\right) & \text { if } \alpha_{0} \leq \tilde{\alpha} \leq \alpha_{0}+\delta,\end{cases} \\
& \bar{f}(\tilde{r}, \tilde{\theta}, \tilde{\alpha})= \begin{cases}f(\tilde{r}, \tilde{\theta}, \tilde{\alpha}), & \text { if } \alpha_{0}-\delta \leq \tilde{\alpha} \leq \alpha_{0}, \\
f\left(\tilde{r}, \tilde{\theta}, 2 \alpha_{0}-\tilde{\alpha}\right) & \text { if } \alpha_{0} \leq \tilde{\alpha} \leq \alpha_{0}+\delta,\end{cases} \\
& \bar{G}_{i}\left(\tilde{r}, \tilde{\theta}, \tilde{\alpha}, \bar{V}, \partial_{\tilde{r}} \bar{V},\left(\partial_{\tilde{\alpha}} \bar{V}\right)^{2}\right)=\left\{\begin{array}{cc}
G_{i}\left(\tilde{r}, \tilde{\theta}, \tilde{\alpha}, \bar{V}, \partial_{\tilde{r}} \bar{V},\left(\partial_{\tilde{\alpha}} \bar{V}\right)^{2}\right) \\
\text { if } \alpha_{0}-\delta \leq \tilde{\alpha} \leq \alpha_{0}, \\
G_{i}\left(\tilde{r}, \tilde{\theta}, 2 \alpha_{0}-\tilde{\alpha}, \bar{V}, \partial_{\tilde{r}} \bar{V},\left(\partial_{\tilde{\alpha}} \bar{V}\right)^{2}\right) \\
\text { if } \alpha_{0} \leq \tilde{\alpha} \leq \alpha_{0}+\delta .
\end{array}\right.
\end{aligned}
$$


Then, $\bar{V} \in C^{1,1-\delta_{0}}(D)$ satisfies

$$
\begin{aligned}
& \bar{a}_{11}(\tilde{r}, \tilde{\theta}, \tilde{\alpha}) \partial_{\tilde{r}}^{2} \bar{V}+2 \bar{a}_{12}(\tilde{r}, \tilde{\theta}, \tilde{\alpha}) \partial_{\tilde{r} \tilde{\alpha}}^{2} \bar{V}+\bar{a}_{22}(\tilde{r}, \tilde{\theta}, \tilde{\alpha}) \partial_{\tilde{\alpha}}^{2} \bar{V}=\bar{f}(\tilde{r}, \tilde{\theta}, \tilde{\alpha}), \\
& \bar{G}_{1}\left(\tilde{r}, \tilde{\theta}, \tilde{\alpha}, \bar{V}, \partial_{\tilde{r}} \bar{V},\left(\partial_{\tilde{\alpha}} \bar{V}\right)^{2}\right)=0 \quad \text { on } \tilde{r}=-x_{1}^{0},
\end{aligned}
$$

where $\bar{a}_{i j}(\tilde{r}, \tilde{\theta}, \tilde{\alpha}), \bar{f}(\tilde{r}, \tilde{\theta}, \tilde{\alpha}) \in C^{1-\delta_{0}}(D), \bar{G}_{1}$ is $C^{1,1-\delta_{0}}(D)$-regular with respect to $(\tilde{r}, \tilde{\theta}, \tilde{\alpha})$, and the nonlinear boundary condition $G_{1}$ is uniformly oblique.

It follows from the regularity assumptions in Theorem 1.5 and the interior regularity theory for uniformly elliptic equations that

$$
\bar{V}(\cdot, \tilde{\theta}, \cdot) \in C^{2,1-\delta_{0}}\left(\left[-x_{1}^{0},-x_{1}^{0}+\delta ; \alpha_{0}-\delta, \alpha_{0}+\delta\right] \backslash\left(-x_{1}^{0}, \alpha_{0}\right)\right)
$$

for each $\tilde{\theta} \in\left[\theta_{0}-\delta, \theta_{0}+\delta\right]$ for suitably small $\delta$. The regularity of $\bar{V}$ at $\left(-x_{1}^{0}, \alpha_{0}\right)$ can also be improved by modifying the proof of [Lieberman and Trudinger 1986, Theorem 1.1]. More precisely, we first consider the boundary value problem

$$
\left\{\begin{array}{l}
\bar{a}_{11}(\tilde{r}, \tilde{\theta}, \tilde{\alpha}) \partial_{\tilde{r}}^{2} U+2 \bar{a}_{12}(\tilde{r}, \tilde{\theta}, \tilde{\alpha}) \partial_{\tilde{r} \tilde{\alpha}}^{2} U+\bar{a}_{22}(\tilde{r}, \tilde{\theta}, \tilde{\alpha}) \partial_{\tilde{r} \tilde{\alpha}}^{2} U=\bar{f}(\tilde{r}, \tilde{\theta}, \tilde{\alpha}), \\
\bar{G}_{1}\left(\tilde{r}, \tilde{\theta}, \tilde{\alpha}, \bar{V}, \partial_{\tilde{r}} U,\left(\partial_{\tilde{\alpha}} U\right)^{2}\right)=0 \quad \text { on } \tilde{r}=-x_{1}^{0}, \\
U=\bar{V} \quad \text { on } \Gamma
\end{array}\right.
$$

where $\Gamma=\left\{(\tilde{r}, \tilde{\alpha}):\left(\tilde{r}+x_{1}^{0}\right)^{2}+\left(\tilde{\alpha}-\alpha_{0}\right)^{2}=\delta^{2}\right\}$. Equation (4-16)' is solved on the half disk $R$, which is surrounded by $\Gamma$ and $\tilde{r}=-x_{1}^{0}$. Indeed, it can be solved by studying a linearized problem as follows: For any $W \in \mathbb{B}$, where

$$
\mathbb{B}=\left\{W \in C^{2,1-\delta_{0}}(R):\left\|W-\left(r_{1}+x_{1}^{0}\right)\right\|_{C^{2,1-\delta_{0}(R)}} \leq M \varepsilon\right\}
$$

with a positive constant $M$ to be chosen, consider

$$
(4-16)^{\prime \prime}\left\{\begin{array}{l}
\bar{a}_{11}(\tilde{r}, \tilde{\theta}, \tilde{\alpha}) \partial_{\tilde{r}}^{2} U+2 \bar{a}_{12}(\tilde{r}, \tilde{\theta}, \tilde{\alpha}) \partial_{\tilde{r} \tilde{\alpha}}^{2} U+\bar{a}_{22}(\tilde{r}, \tilde{\theta}, \tilde{\alpha}) \partial_{\tilde{\alpha}}^{2} U=\bar{f} \quad \text { on } R, \\
U=\bar{V} \quad \text { on } \Gamma \\
\bar{g}_{1}(\tilde{r}, \tilde{\theta}, \tilde{\alpha}) \partial_{\tilde{r}} U+\bar{g}_{2}(\tilde{r}, \tilde{\theta}, \tilde{\alpha}) \partial_{\tilde{\alpha}} U=\bar{g}(\tilde{r}, \tilde{\theta}, \tilde{\alpha})
\end{array}\right.
$$

where

$$
\begin{gathered}
\bar{g}_{1}(\tilde{r}, \tilde{\theta}, \tilde{\alpha})=\int_{0}^{1}\left(\partial _ { \partial _ { \tilde { r } } \overline { V } } \overline { G } _ { 1 } \left(\tilde{r}, \tilde{\theta}, \tilde{\alpha}, t\left(r_{1}+x_{1}^{0}\right)+(1-t) \bar{V}\right.\right. \\
\left.\left.\quad(1-t) \partial_{\tilde{r}} W,(1-t)\left(\partial_{\tilde{\alpha}} W\right)^{2}\right)\right) d t, \\
\bar{g}_{2}(\tilde{r}, \tilde{\theta}, \tilde{\alpha})=\int_{0}^{1}\left(\partial _ { ( \partial _ { \tilde { \alpha } } \overline { V } ) ^ { 2 } } \overline { G } _ { 1 } \left(\tilde{r}, \tilde{\theta}, \tilde{\alpha}, t\left(r_{1}+x_{1}^{0}\right)+(1-t) \bar{V}\right.\right. \\
\left.\left.(1-t) \partial_{\tilde{r}} W,(1-t)\left(\partial_{\tilde{\alpha}} W\right)^{2}\right)\right) d t \partial_{\tilde{\alpha}} W, \\
\bar{g}(\tilde{r}, \tilde{\theta}, \tilde{\alpha})=-\int_{0}^{1}\left(\partial _ { \overline { V } } \overline { G } _ { 1 } \left(\tilde{r}, \tilde{\theta}, \tilde{\alpha}, t\left(r_{1}+x_{1}^{0}\right)+(1-t) \bar{V},\right.\right. \\
\left.\left.(1-t) \partial_{\tilde{r}} W,(1-t)\left(\partial_{\tilde{\alpha}} W\right)^{2}\right)\right) d t\left(\bar{V}-\left(r_{1}+x_{1}^{0}\right)\right) .
\end{gathered}
$$


Note that $\bar{V} \in C^{2,1-\delta_{0}}(\Gamma)$ due to the interior regularity. So one can apply the standard theory for second order uniformly elliptic equations with mixed-type boundary conditions to derive the existence and a uniform estimate for the solution to the problem (4-16)"; see [Lieberman and Trudinger 1986; Gilbarg and Trudinger 2001; Nazarov and Plamenevsky 1994]. Then it can be checked that there exists a suitable $M$ so that the Schauder fixed point theorem can be applied to yield a solution $U \in \mathbb{B}$ to the problem (4-16)' for suitably small $\varepsilon$. Note that we have used the fact that $V=r_{1}+x_{1}^{0}$ satisfies both the equation and the boundary condition in (4-16). It follows from the uniqueness of the solution to the problem (4-16)' in the class $C^{1,1-\delta_{0}}(R) \cap C^{2,1-\delta_{0}}(\stackrel{\circ}{R})$ (here $\stackrel{\circ}{R}$ denotes the interior of the close disk $R$ ) that $\bar{V}(\cdot, \tilde{\theta}, \cdot)=U(\cdot, \tilde{\theta}, \cdot)$ for each $\tilde{\theta} \in\left[\theta_{0}-\delta / 2, \theta_{0}+\delta / 2\right]$. Thus we have shown

$$
\left\|\bar{V}(\cdot, \tilde{\theta}, \cdot)-\left(r_{1}+x_{1}^{0}\right)\right\|_{C^{2,1-\delta_{0}(R)}} \leq C \varepsilon
$$

for each $\tilde{\theta} \in\left[\theta_{0}-\delta / 2, \theta_{0}+\delta / 2\right]$.

Next, we improve the regularity of $\bar{V}$ with respect to $\tilde{\theta}$. Let $\delta_{0}^{\prime} \in\left(\delta_{0}, 1\right)$ be a fixed constant. For any $\tilde{\theta}_{1}$ and $\tilde{\theta}_{2}$ in $\left[\theta_{0}-\delta / 2, \theta_{0}+\delta / 2\right]$ with $\tilde{\theta}_{1} \neq \tilde{\theta}_{2}$, it follows from (4-16) that $W_{1}\left(\tilde{r}, \tilde{\theta}_{1}, \tilde{\theta}_{2}, \tilde{\alpha}\right)=\left(\bar{V}\left(\tilde{r}, \tilde{\theta}_{1}, \tilde{\alpha}\right)-\bar{V}\left(\tilde{r}, \tilde{\theta}_{2}, \tilde{\alpha}\right)\right)\left|\tilde{\theta}_{1}-\tilde{\theta}_{2}\right|^{\delta_{0}^{\prime}-1}$ satisfies

$$
\bar{a}_{11}(\tilde{r}, \tilde{\theta}, \tilde{\alpha}) \partial_{\tilde{r}}^{2} W_{1}+2 \bar{a}_{12}(\tilde{r}, \tilde{\theta}, \tilde{\alpha}) \partial_{\tilde{r} \tilde{\alpha}}^{2} W_{1}+\bar{a}_{22}(\tilde{r}, \tilde{\theta}, \tilde{\alpha}) \partial_{\tilde{\alpha}}^{2} W_{2}=f_{0}(\tilde{r}, \tilde{\theta}, \tilde{\alpha})
$$

and

$$
\begin{aligned}
g_{11}\left(\tilde{r}, \tilde{\theta}_{1}, \tilde{\theta}_{2}, \tilde{\alpha}\right) \partial_{\tilde{r}} W_{1}+g_{12}\left(\tilde{r}, \tilde{\theta}_{1}, \tilde{\theta}_{2}, \tilde{\alpha}\right) \partial_{\tilde{\alpha}} W_{1}+g_{13}\left(\tilde{r}, \tilde{\theta}_{1}, \tilde{\theta}_{2}, \tilde{\alpha}\right) W_{1} \\
=g_{10}\left(\tilde{r}, \tilde{\theta}_{1}, \tilde{\theta}_{2}, \tilde{\alpha}\right)
\end{aligned}
$$

on $\tilde{r}=-x_{1}^{0}$, with $f_{0}\left(\cdot, \tilde{\theta}_{1}, \tilde{\theta}_{2}, \cdot\right) \in C^{\delta^{\prime}-\delta_{0}}$ and $g_{1 j}\left(\cdot, \tilde{\theta}_{1}, \tilde{\theta}_{2}, \cdot\right) \in C^{1, \delta_{0}^{\prime}-\delta}$.

For $(\tilde{r}, \tilde{\alpha}) \in\left[-x_{1}^{0},-x_{1}^{0}+\delta ; \alpha_{0}-\delta, \alpha_{0}+\delta\right]$, it follows from the maximum principle that

$$
\begin{aligned}
\left|W_{1}\left(\tilde{r}, \tilde{\theta}_{1}, \tilde{\theta}_{2}, \tilde{\alpha}\right)\right| \leq C( & \left\|W_{1}\left(-x_{1}^{0}+\delta, \tilde{\theta}_{1}, \tilde{\theta}_{2}, \cdot\right)\right\|_{L^{\infty}\left[\alpha_{0}-\delta, \alpha_{0}+\delta\right]} \\
+ & \left\|W_{1}\left(\cdot, \tilde{\theta}_{1}, \tilde{\theta}_{2}, \alpha_{0} \pm \delta\right)\right\|_{L^{\infty}\left[-x_{1}^{0},-x_{1}^{0}+\delta\right]} \\
& \left.+\left\|f_{0}\left(\cdot, \tilde{\theta}_{1}, \tilde{\theta}_{2}, \cdot\right)\right\|_{L^{\infty}}+\left\|g_{10}\left(\cdot, \tilde{\theta}_{1}, \tilde{\theta}_{2}, \cdot\right)\right\|_{L^{\infty}}\right) \leq C \varepsilon .
\end{aligned}
$$

It follows from standard estimates for second order elliptic equations that

$$
\begin{aligned}
& \left\|W_{1}\left(\cdot, \tilde{\theta}_{1}, \tilde{\theta}_{2}, \cdot\right)\right\|_{C^{2, \delta_{0}^{\prime}-\delta_{0}}\left[-x_{1}^{0},-x_{1}^{0}+\delta / 2 ; \alpha_{0}-\delta / 2, \alpha_{0}+\delta / 2\right]} \\
& \leq C\left(\left\|W_{1}\left(\cdot, \tilde{\theta}_{1}, \tilde{\theta}_{2}, \cdot\right)\right\|_{L^{\infty}\left[-x_{1}^{0},-x_{1}^{0}+\delta ; \alpha_{0}-\delta, \alpha_{0}+\delta\right]}\right. \\
& \left.+\left\|f_{0}\left(\cdot, \tilde{\theta}_{1}, \tilde{\theta}_{2}, \cdot\right)\right\|_{C^{\delta^{\prime}-\delta_{0}}}+\left\|g_{10}\left(\cdot, \tilde{\theta}_{1}, \tilde{\theta}_{2}, \cdot\right)\right\|_{C^{1, \delta_{0}^{\prime}-\delta_{0}}}\right) .
\end{aligned}
$$

Hence $\bar{V}(\tilde{r}, \tilde{\theta}, \tilde{\alpha})$ belongs to

$$
C^{1-\delta_{0}^{\prime}}\left(\left[\theta_{0}-\delta / 2, \theta_{0}+\delta / 2\right], C^{2, \delta_{0}^{\prime}-\delta_{0}}\left[-x_{1}^{0},-x_{1}^{0}+\delta / 2 ; \tilde{\alpha}_{0}-\delta / 2, \tilde{\alpha}_{0}+\delta / 2\right]\right) .
$$


It follows from this and (4-13) that $\bar{V}(\tilde{r}, \tilde{\theta}, \tilde{\alpha}) \in C^{2,1-\delta_{0}^{\prime}}(D)$.

A similar result holds near $\tilde{l}_{2}$. This yields

$$
V(\tilde{r}, \tilde{\theta}, \tilde{\alpha}) \in C^{2,1-\delta_{0}^{\prime}}\left(\bar{Q}_{+}\right) \quad \text { and } \quad\left\|V-\left(r_{1}+x_{1}^{0}\right)\right\|_{C^{2,1-\delta_{0}^{\prime}}\left(\bar{Q}_{+}\right)} \leq C \varepsilon .
$$

Consequently, Proposition 4.1 is proved.

Next, we prove $\partial_{\theta} \varphi_{+}\left(r, \theta, \alpha_{0}\right) \equiv 0$ on the intersection curve $\tilde{l}_{1}=\Sigma \cap\left\{\alpha=\alpha_{0}\right\}$; this will be used to find the value of $\partial_{\tilde{\theta}} V(\tilde{r}, \tilde{\theta}, \tilde{\alpha})$ at the fixed point $\left(-x_{1}^{0}, \tilde{\theta}_{0}, \alpha_{0}\right)$.

Proposition 4.3. We have $\partial_{\theta} \varphi_{+}\left(r, \theta, \alpha_{0}\right) \equiv 0$ on the intersection curve

$$
\tilde{l}_{1}=\left\{\left(-x_{1}^{0}, \theta, \alpha_{0}\right): 0 \leq \theta<2 \pi\right\} .
$$

Proof. First we show that the shock surface $\Sigma$ is perpendicular to the fixed boundary $\Pi_{2}$.

Indeed, it follows from the continuity of $\varphi$ on $\Sigma$ in (4-4)-(4-6) that

$$
\varphi_{+}\left(r(\theta, \alpha)-x_{1}^{0}, \theta, \alpha\right)=\varphi_{-}\left(r(\theta, \alpha)-x_{1}^{0}\right) .
$$

Thus one can derive easily that

$$
\begin{aligned}
\partial_{r} \varphi_{+}\left(r(\theta, \alpha)-x_{1}^{0}, \theta, \alpha\right) \partial_{\alpha} r(\theta, \alpha)+\partial_{\alpha} \varphi_{+}( & \left.(\theta, \alpha)-x_{1}^{0}, \theta, \alpha\right) \\
& =\partial_{r} \varphi_{-}\left(r(\theta, \alpha)-x_{1}^{0}, \theta, \alpha\right) \partial_{\alpha} r(\theta, \alpha) .
\end{aligned}
$$

It follows from $\partial_{\alpha} \varphi_{+}\left(\theta, \alpha_{0}\right)=0$ and $\left[\partial_{r} \varphi\right] \neq 0$ that $\partial_{\alpha} r\left(\theta, \alpha_{0}\right) \equiv 0$. This implies that $\Sigma$ is perpendicular to the fixed boundary $\Pi_{2}$.

To show that $\partial_{\theta} \varphi_{+}\left(r, \theta, \alpha_{0}\right) \equiv 0$ on $\tilde{l}_{1}$, we note that due to the symmetry of $\varphi_{-}$, the boundary condition in (4-4)-(4-6) becomes

$$
\begin{aligned}
\left(\left(\partial_{r} \varphi_{+} \partial_{r}+\frac{1}{r^{2} \sin ^{2} \alpha} \partial_{\theta} \varphi_{+} \partial_{\theta}+\frac{1}{r^{2}} \partial_{\alpha} \varphi_{+} \partial_{\alpha}\right)\left(\varphi_{+}-\varphi_{-}\right)\right) H_{+} & \\
& -\partial_{r} \varphi_{-} \partial_{r}\left(\varphi_{+}-\varphi_{-}\right) H_{-}=0
\end{aligned}
$$

on $\Sigma$.

Since it follows from the assumptions in Theorem 1.5 that $\varphi_{+} \in C^{2}\left(\Omega_{+}\right)$and $\left|\nabla^{2} \varphi_{+}(r(\theta, \alpha), \theta, \alpha)\right| \leq C \varepsilon /\left|\alpha-\alpha_{0}\right|^{\delta_{0}}$ for $\alpha \neq \alpha_{0}$ and near the curve $\tilde{l}_{1}$ and also since $r(\theta, \alpha) \in C^{1,1-\delta_{0}}\left[0,2 \pi ; \alpha_{0} / 2, \alpha_{0}\right]$ and $\left|\partial_{\alpha} r(\theta, \alpha)\right| \leq C \varepsilon\left|\alpha-\alpha_{0}\right|^{1-\delta_{0}}$ due to (4-17), we arrive at

$$
\lim _{\alpha \rightarrow \alpha_{0}}\left(\nabla^{2} \varphi_{+}\left(r(\theta, \alpha)-x_{1}^{0}, \theta, \alpha\right) \partial_{\alpha} r(\theta, \alpha)\right)=0 .
$$

Differentiating (4-18) with respect to $\alpha$, and using (4-19) together with the boundary condition $\partial_{\alpha} \varphi_{+}\left(r, \theta, \alpha_{0}\right) \equiv 0$ yield, on the intersection curve $\tilde{l}_{1}$, that

$$
\left(2 H_{+}-\frac{\left(\partial_{\theta} \varphi_{+}\right)^{2} H_{+}^{\prime}}{r^{2} \sin ^{2} \alpha_{0}}\right) \frac{\cos \alpha_{0}}{r^{2} \sin ^{3} \alpha_{0}}\left(\partial_{\theta} \varphi_{+}\right)^{2}\left(r, \theta, \alpha_{0}\right) \equiv 0,
$$


where we have used Proposition 4.1. This yields $\partial_{\theta} \varphi_{+}\left(r, \theta, \alpha_{0}\right) \equiv 0$ on $\tilde{l}_{1}$. It remains to derive the equation for $\tilde{l}_{1}$. By $\varphi_{+}\left(r(\theta, \alpha)-x_{1}^{0}, \theta, \alpha\right)=\varphi_{-}\left(r(\theta, \alpha)-x_{1}^{0}\right)$, we have on $\alpha=\alpha_{0}$ that

$$
\begin{aligned}
\partial_{r} \varphi_{+}\left(r\left(\theta, \alpha_{0}\right)-x_{1}^{0}, \theta, \alpha_{0}\right) \partial_{\theta} r\left(\theta, \alpha_{0}\right)+\partial_{\theta} \varphi_{+}\left(r\left(\theta, \alpha_{0}\right)-x_{1}^{0}, \theta, \alpha_{0}\right) \\
=\partial_{r} \varphi_{-}\left(r\left(\theta, \alpha_{0}\right)-x_{1}^{0}, \theta, \alpha_{0}\right) \partial_{\theta} r\left(\theta, \alpha_{0}\right) .
\end{aligned}
$$

It follows from $\partial_{\theta} \varphi_{+}\left(r\left(\theta, \alpha_{0}\right)-x_{1}^{0}, \theta, \alpha_{0}\right)=0$ and $\left[\partial_{r} \varphi\right] \neq 0$ that $\partial_{\theta} r\left(\theta, \alpha_{0}\right) \equiv 0$. This implies $r\left(\theta, \alpha_{0}\right) \equiv r\left(\tilde{\theta}_{0}, \alpha_{0}\right)=0$. Thus $\tilde{l}_{1}$ is a circle given by

$$
\left\{\left(-x_{1}^{0}, \theta, \alpha_{0}\right): 0 \leq \theta<2 \pi\right\} .
$$

We now show $\varphi_{+}(x)$ is independent of $\theta$ under the assumptions of Theorem 1.5. Proposition 4.4. $\left(\varphi_{+}(r, \theta, \alpha), r(\theta, \alpha)\right)$ is independent of the variable $\theta$.

Proof. First, it will be more convenient here to use cylindrical coordinates (since $\alpha=0$ is singular for the transformation (4-1)). These are

$$
x_{1}=x_{1}, \quad x_{2}=\rho \cos \theta, \quad x_{3}=\rho \sin \theta
$$

with $\rho=\left(x_{2}^{2}+x_{3}^{2}\right)^{1 / 2}$ and $0 \leq \theta<2 \pi$.

Then the Equation (1-3) for $\varphi_{+}$can be rewritten as

$$
\begin{aligned}
& \left(\left(\partial_{1} \varphi_{+}\right)^{2}-c^{2}\left(H_{+}\right)\right) \partial_{1}^{2} \varphi_{+}+2 \partial_{1} \varphi_{+} \partial_{\rho} \varphi_{+} \partial_{1 \rho}^{2} \varphi_{+}+\frac{2 \partial_{1} \varphi_{+} \partial_{\theta} \varphi_{+}}{\rho^{2}} \partial_{1 \theta}^{2} \varphi_{+} \\
& \quad+\left(\left(\partial_{\rho} \varphi_{+}\right)^{2}-c^{2}\left(H_{+}\right)\right) \partial_{\rho}^{2} \varphi_{+}+\frac{2 \partial_{\rho} \varphi_{+} \partial_{\theta} \varphi_{+}}{\rho^{2}} \partial_{\rho \theta}^{2} \varphi_{+} \\
& \quad+\frac{1}{\rho^{2}}\left(\frac{\left(\partial_{\theta} \varphi_{+}\right)^{2}}{\rho^{2}}-c^{2}\left(H_{+}\right)\right) \partial_{\theta}^{2} \varphi_{+}-\frac{c^{2}\left(H_{+}\right) \partial_{\rho} \varphi_{+}}{\rho}-\frac{\partial_{\rho} \varphi_{+}\left(\partial_{\theta} \varphi_{+}\right)^{2}}{\rho^{3}}=0
\end{aligned}
$$

where $H_{+}=H\left(C_{0}-\frac{1}{2}\left(\left(\partial_{1} \varphi_{+}\right)^{2}+\left(\partial_{\rho} \varphi_{+}\right)^{2}+\left(1 / \rho^{2}\right)\left(\partial_{\theta} \varphi_{+}\right)^{2}\right)\right)$ and $\partial_{i}=\partial_{x_{i}}$ for $i=1,2,3$.

Suppose that the shock surface $\Sigma$ is given by $x_{1}=\Xi(\rho, \theta)$. By the assumption of Theorem 1.5,

$$
\Xi\left(\left(\left(\tilde{x}_{2}^{0}\right)^{2}+\left(\tilde{x}_{3}^{0}\right)^{2}\right)^{1 / 2}, \tilde{\theta}_{0}\right)=\tilde{x}_{1}^{0} .
$$

The boundary conditions are $\varphi_{+}=\varphi_{-}$on $\Sigma, \operatorname{tg} \alpha_{0} \partial_{1} \varphi_{+}-\partial_{\rho} \varphi_{+}=0$ on $\Pi_{2}$, $\left[\partial_{1} \varphi H\right] \partial_{1}\left(\varphi_{+}-\varphi_{-}\right)-\partial_{\rho} \varphi_{-}\left\{\partial_{\rho}\left(\varphi_{+}-\varphi_{-}\right)\right\} H_{-}$

$$
+\left\{\left(\partial_{\rho} \varphi_{+} \partial_{\rho}+\left(1 / \rho^{2}\right) \partial_{\theta} \varphi_{+} \partial_{\theta}\right)\left(\varphi_{+}-\varphi_{-}\right)\right\} H_{+}=0 \quad \text { on } \Sigma,
$$

$H\left(C_{0}-\frac{1}{2}\left(\left(\partial_{1} \varphi_{+}\right)^{2}+\left(\partial_{\rho} \varphi_{+}\right)^{2}+\left(1 / \rho^{2}\right)\left(\partial_{\theta} \varphi_{+}\right)^{2}\right)\right)=\rho_{+}$

$$
\text { on }\left(\rho^{2}+\left(x_{1}-x_{1}^{0}\right)^{2}\right)^{1 / 2}=r_{1},
$$


Now we will rewrite the problem (4-20) with the given shock surface and boundary conditions using the partial hodograph transformation

$$
\begin{aligned}
& \tilde{x}_{1}=1-\left(1-x_{1}\right) /\left(1-x_{1}+\varphi_{-}\left(x_{1}, \rho\right)-\varphi_{+}\left(x_{1}, \rho, \theta\right)\right), \\
& \tilde{\rho}=\rho /\left(\left(x_{1}-x_{1}^{0}\right) \operatorname{tg} \alpha_{0}\right), \\
& \tilde{\theta}=\theta,
\end{aligned}
$$

and $V\left(\tilde{x}_{1}, \tilde{\rho}, \tilde{\theta}\right)=1-x_{1}+\varphi_{-}\left(x_{1}, \rho\right)-\varphi_{+}\left(x_{1}, \rho, \theta\right)$.

Then as in Section 2, it follows from a direct computation and the assumptions in Theorem 1.5 that $w=\partial_{\tilde{\theta}} V$ satisfies

$$
\left\{\begin{aligned}
\sum_{i, j=1,2,3} a_{i j}(y) \partial_{y_{i} y_{j}}^{2} w & \\
+\sum_{i=1,2,3} b_{i}(y) \partial_{y_{i}} w+c(y) w=0 & \text { for } y \in Q_{+} \\
\partial_{y_{1}} w+\sum_{i=2,3} \gamma_{1 i}(y) \partial_{y_{i}} w+d_{1}(y) w=0 & \text { on } y_{1}=0, \\
\partial_{y_{1}} w+\sum_{i=2,3} \gamma_{2 i}(y) \partial_{y_{i}} w+d_{2}(y) w=0 & \text { on } y_{1}=1, \\
\sum_{i=1,2,3} \gamma_{3 i}(y) \partial_{y_{i}} w+d_{3}(y) w=0 & \text { on } y_{2}=1,
\end{aligned}\right.
$$

where $y=\left(y_{1}, y_{2}, y_{3}\right)=\left(\tilde{x}_{1}, \tilde{\rho}, \tilde{\theta}\right), Q_{+}=\left\{y: 0<y_{1}<1,0 \leq y_{2}<1,0 \leq y_{3}<2 \pi\right\}$. Let $\delta>0$ be a suitably small fixed constant with $\delta<1$ and $Q_{+}^{\delta}=Q_{+} \cap\left\{y_{2} \geq \delta\right\}$. Then $b_{i}(y), c(y) \in C^{1}\left(\bar{Q}_{+}^{\delta} \backslash \bigcup_{i=1}^{2} \Gamma_{i}\right)$ and $\gamma_{i j}(y), d_{i}(y) \in C^{1}\left(\bar{Q}_{+}^{\delta} \backslash \bigcup_{i=1}^{2} \Gamma_{i}\right) \cap C^{1-\delta_{0}}\left(\bar{Q}_{+}^{\delta}\right)$. On $\bar{Q}_{+}^{\delta}$, these coefficients satisfy the estimates

$$
\begin{array}{cl}
\sum_{i=1,2,3}\left|\nabla_{y}^{k} b_{i}(y)\right|+\left|\nabla_{y}^{k} c(y)\right| \leq C \varepsilon / R^{k+\delta_{0}} & \text { for } k=0,1, \\
\sum_{j}\left\|\gamma_{i j}\right\|_{C^{1-\delta_{0}}}+\sum_{j=1,2,3}\left\|d_{j}\right\|_{C^{1-\delta_{0}}} \leq C \varepsilon & \text { for } i=1,2, \\
\sum_{j}\left|\nabla_{X}^{k} \gamma_{i j}\right|+\sum_{j=1,2,3}\left|\nabla_{X}^{k} d_{j}\right| \leq C \varepsilon / R^{k-1+\delta_{0}} & \text { for } i=1,2 \text { and } k=1,2, \\
\sum_{j=2,3}\left|\nabla_{X}^{k}\left(\gamma_{3 j}-X_{j}\right)\right|+\left|\nabla_{X}^{k} \gamma_{31}\right| \leq C \varepsilon / R^{k-1+\delta_{0}} & \text { for } k=1,2, \\
\left\|\gamma_{31}-\operatorname{tg} \alpha_{0}\right\|_{C^{1-\delta_{0}}}+\left\|\gamma_{32}+1\right\|_{C^{1-\delta_{0}}}+\left\|\gamma_{33}\right\|_{C^{1-\delta_{0}}} \leq C \varepsilon,
\end{array}
$$

where $\Gamma_{1}=\left\{y: y_{1}=0, y_{2}=1,0 \leq y_{3}<2 \pi\right\}, \Gamma_{2}=\left\{y: y_{1}=1, y_{2}=1,0 \leq y_{3}<2 \pi\right\}$, and $R=y_{1}\left(1-y_{1}\right)+1-y_{2}^{2}$. Near $y_{2}=0$, the above coefficients are $C^{3}$-smooth with respect to the coordinates $X=\left(X_{1}, X_{2}, X_{3}\right)$ with $X_{1}=y_{1}, X_{2}=y_{2} \cos y_{3}$, and $X_{3}=y_{2} \sin y_{3}$; for details, see the treatments of the transformation (2-3).

Next we determine the value $w\left(0,1, \tilde{\theta}_{0}\right)$. 
Since $\partial_{\theta} V\left(\tilde{x}_{1}, \tilde{\rho}, \tilde{\theta}\right)=-\partial_{\theta} \varphi_{+}\left(x_{1}, \rho, \theta\right)$, it follows from Proposition 4.3 that

$$
\partial_{\theta} V\left(\tilde{x}_{1}, \tilde{\rho}, \tilde{\theta}\right) \equiv 0 \quad \text { on } \Gamma_{1} \text {. }
$$

Because $\tilde{x}_{1}=1-1-x_{1} / V$ and by (4-23), we have $\partial_{\theta} \tilde{x}_{1}=\left(1-x_{1}\right) / V^{2} \partial_{\theta} V \equiv 0$ on $\Gamma_{1}$. Thus $\partial_{\tilde{\theta}} V=\partial_{\theta} V-\partial_{\tilde{x}_{1}} V \partial_{\theta} \tilde{x}_{1} \equiv 0$ on $\Gamma_{1}$. This implies

$$
w\left(0,1, \tilde{\theta}_{0}\right)=0 .
$$

By analogous arguments for Theorem 1.1, we can show that the problem (4-22) together with (4-24) has a unique solution $w \equiv 0$. It follows from $\partial_{\theta} V=V w /(V-$ $\left.\left(1-\tilde{x}_{1}\right) \partial_{\tilde{x}_{1}} V\right)$ that $\partial_{\theta} V=\partial_{\theta} \varphi_{+} \equiv 0$ in $\Omega_{+}$.

Since Proposition 4.4 says $\left(\varphi_{+}(r, \theta, \alpha), r(\theta, \alpha)\right) \equiv\left(\varphi_{+}(r, \alpha), r(\alpha)\right)$, Equation (4-2) with (4-3)-(4-6) can be simplified as

$$
(4-25) \begin{cases}c^{2}\left(H_{+}\right)\left(\frac{1}{r^{2}} \partial_{r}\left(r^{2} \partial_{r} \varphi_{+}\right)+\frac{1}{r^{2} \sin \alpha} \partial_{\alpha}\left(\sin \alpha \partial_{\alpha} \varphi_{+}\right)\right) & -\frac{1}{2}\left(\partial_{r} \varphi_{+} \partial_{r}+\frac{1}{r^{2}} \partial_{\alpha} \varphi_{+} \partial_{\alpha}\right)\left(\left|\nabla \varphi_{+}\right|^{2}\right)=0, \\ r\left(\tilde{\theta}_{0}, \alpha_{0}\right)=0, & \text { on } \Sigma, \\ \varphi_{+}=\varphi_{-} & \\ \left(\left(\partial_{r} \varphi_{+} \partial_{r}+\frac{1}{r^{2}} \partial_{\alpha} \varphi_{+} \partial_{\alpha}\right)\left(\varphi_{+}-\varphi_{-}\right)\right) H_{+} & \\ -\left(\left(\partial_{r} \varphi_{-} \partial_{r}+\frac{1}{r^{2}} \partial_{\alpha} \varphi_{-} \partial_{\alpha}\right)\left(\varphi_{+}-\varphi_{-}\right)\right) H_{-}=0 & \text { on } \Sigma, \\ H\left(C_{0}-\frac{1}{2}\left(\left(\partial_{r} \varphi_{+}\right)^{2}+\frac{1}{r^{2}}\left(\partial_{\alpha} \varphi_{+}\right)^{2}\right)\right)=\rho_{+} & \text {on } r=\left(1-x_{1}^{0}\right) \sec \alpha_{0}, \\ \partial_{\alpha} \varphi_{+}=0 & \text { on } \Pi_{2}\end{cases}
$$

with $H_{+}=H\left(C_{0}-(1 / 2)\left(\left(\partial_{r} \varphi_{+}\right)^{2}+\left(1 / r^{2}\right)\left(\partial_{\alpha} \varphi_{+}\right)^{2}\right)\right)$.

Next we show that

$$
\lim _{\alpha \rightarrow 0+} \partial_{\alpha} \varphi_{+}=0 .
$$

Indeed, it follows from direct computations and the assumptions on the regularity of $\varphi_{+}(x)$ in $\Omega_{+}$that $\partial_{r} \varphi_{+}, \partial_{\alpha} \varphi_{+}, \partial_{r}^{2} \varphi_{+}, \partial_{r \alpha}^{2} \varphi_{+}$and $\partial_{\alpha}^{2} \varphi_{+}$are uniformly bounded near $\alpha=0$ (that is $x_{1}$-axis in the interior of $\Omega_{+}$). In addition, the first equation in (4-25) shows that $\cos \alpha \partial_{\alpha} \varphi_{+}=\left(\frac{\left(r^{2} \partial_{r} \varphi_{+} \partial_{r}+\partial_{\alpha} \varphi_{+} \partial_{\alpha}\right)\left(\left|\nabla \varphi_{+}\right|^{2}\right)}{2 c^{2}\left(H_{+}\right)}-r^{2} \partial_{r}^{2} \varphi_{+}-2 r \partial_{r} \varphi_{+}-\partial_{\alpha}^{2} \varphi_{+}\right) \sin \alpha$.

Consequently, $\lim _{\alpha \rightarrow 0+} \partial_{\alpha} \varphi_{+}=0$ holds. 
Now we show that $\left(\varphi_{+}(r, \alpha), r(\alpha)\right)$ is in fact independent of $\alpha$ under the assumptions of Theorem 1.5.

Proposition 4.5. Under the assumptions in Theorem 1.5, if $\left(\varphi_{+}(r, \alpha), r(\alpha)\right)$ solves problem (4-25), then it is independent of the variable $\alpha$ for $\alpha \in\left[0, \alpha_{0}\right]$.

Remark 4.6. The idea of the proof of Proposition 4.5 is based on the following observation:

Let $u$ be a solution to the Laplace equation $\Delta u=0$ in $\mathbb{R}^{3}$. If $u$ is independent of the variable $\theta$, then it satisfies

$$
\partial_{r}^{2} u+\frac{\partial_{\alpha}^{2} u}{r^{2}}+\frac{2 \partial_{r} u}{r}+\frac{\partial_{\alpha} u}{r^{2} \operatorname{tg} \alpha}=0 \text { for } r>0 \text { and } 0<\alpha<\pi
$$

Setting $w=\partial_{\alpha} u$, we have

$$
\partial_{r}^{2} w+\frac{\partial_{\alpha}^{2} w}{r^{2}}+\frac{2 \partial_{r} w}{r}+\frac{1}{r^{2} \operatorname{tg} \alpha} \partial_{\alpha} w-\frac{1}{r^{2} \sin ^{2} \alpha} w=0 \quad \text { for } r>0 \text { and } 0<\alpha<\pi
$$

This equation for $w$ has a maximum principle since the coefficient of $w$ is negative for $0<\alpha<\pi$.

Proof of Proposition 4.5. We will use the same notations as in the proof of Proposition 4.1. Differentiating the first equation in (4-14) and (4-15) with respect to $\tilde{\alpha}$ and noting that $V \in C^{2,1-\delta_{0}}\left(\bar{Q}_{+}\right) \cap C^{3}\left(Q_{+} \backslash\{\alpha=0\}\right)$ (the regularity of $V$ has been given in the proof of Proposition 4.1) is independent of $\theta$, then we get that $w=\partial_{\tilde{\alpha}} V$ satisfies

$$
\begin{aligned}
& a_{11}(\tilde{r}, \tilde{\alpha}) \partial_{\tilde{r}}^{2} w+2 a_{12}(\tilde{r}, \tilde{\alpha}) \partial_{\tilde{r} \tilde{\alpha}}^{2} w+a_{22}(\tilde{r}, \tilde{\alpha}) \partial_{\tilde{\alpha}}^{2} w+2 \partial_{\tilde{\alpha}} a_{12} \partial_{\tilde{r}} w \\
& +\partial_{\tilde{\alpha}} a_{22} \partial_{\tilde{\alpha}} w+\frac{\partial_{\tilde{\alpha}} a_{11}}{a_{11}}\left(f-2 a_{12} \partial_{\tilde{r}} w-a_{22} \partial_{\tilde{\alpha}} w\right)=\partial_{\tilde{\alpha}} f
\end{aligned}
$$

where $a_{i j}(\tilde{r}, \tilde{\alpha})$ and $f(\tilde{r}, \tilde{\alpha})$ can be obtained from $a_{i j}(\tilde{r}, \tilde{\theta}, \tilde{\alpha})$ and $f(\tilde{r}, \tilde{\theta}, \tilde{\alpha})$ by setting $p_{i}(\tilde{r}, \tilde{\theta}, \tilde{\alpha}) \equiv 0$ in the proof procedure of Proposition 4.1 and dropping all the terms involving derivatives with respect to $\theta$ (or $\tilde{\theta}$ ).

Then, in terms of the concrete expressions of $\left(\partial_{\tilde{\alpha}} a_{11} / a_{11}\right) f, \partial_{\tilde{\alpha}} a_{11}, \partial_{\tilde{\alpha}} f$, and the special term $\left(1 / r^{2} \operatorname{tg} \alpha\right) \partial_{\alpha} \varphi_{+}$in $f(\tilde{r}, \tilde{\alpha})$, one can derive from (4-25)-(4-27), 
Proposition 4.1, and a direct but tedious computation that $w=\partial_{\tilde{\alpha}} V$ satisfies

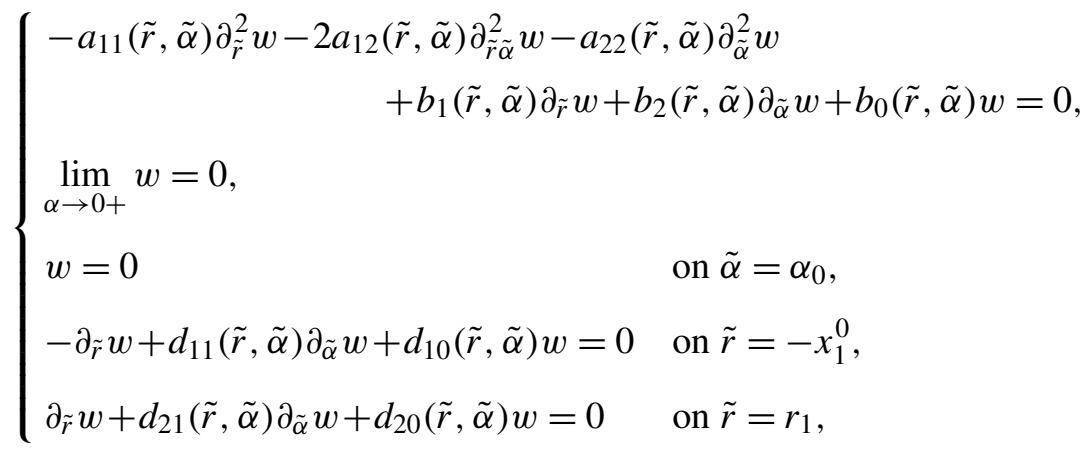

where

$$
\begin{aligned}
& b_{i}(\tilde{r}, \tilde{\alpha}) \in C^{1}\left(-x_{1}^{0}, r_{1} ; 0, \alpha_{0}\right) \quad \text { for } i=0,1,2 \text {, } \\
& a_{i j}(\tilde{r}, \tilde{\alpha}), d_{i j}(\tilde{r}, \tilde{\alpha}) \in C^{1}\left[-x_{1}^{0}, r_{1} ; 0, \alpha_{0}\right] \text {, } \\
& \left|d_{i j}(\tilde{r}, \tilde{\alpha})\right| \leq C(\varepsilon+1 / r) \text {, } \\
& \left|b_{1}(\tilde{r}, \tilde{\alpha})\right| \leq C, \\
& b_{0}(\tilde{r}, \tilde{\alpha})=\frac{1}{r^{2} \sin ^{2} \alpha}(1+O(1 / r)+O(\varepsilon)),
\end{aligned}
$$

$b_{2}(\tilde{r}, \tilde{\alpha})$ contains the singular factor $1 /(\operatorname{tg} \tilde{\alpha})$, and the generic constant $C$ depends only on $\rho_{0}$ and $q_{-}$for large $X_{0}$.

In addition, there exists a constant $C_{0}>0$ which is independent of $\varepsilon$ such that for any $\xi=\left(\xi_{1}, \xi_{2}\right) \in \mathbb{R}^{2}$, we have $\sum_{i, j=1,2} a_{i j}(\tilde{r}, \tilde{\alpha}) \xi_{i} \xi_{j} \geq C_{0}|\xi|^{2}$.

Set $w(\tilde{r}, \tilde{\alpha})=\exp \left(\left(\varepsilon^{1 / 2}+X_{0}^{-1 / 2}\right)\left(\tilde{r}-\left(r_{1}-x_{1}^{0}\right) / 2\right)^{2}\right) \tilde{w}(\tilde{r}, \tilde{\alpha})$. It follows from (4-28) that

$$
\begin{cases}-a_{11}(\tilde{r}, \tilde{\alpha}) \partial_{\tilde{r}}^{2} \tilde{w}-2 a_{12}(\tilde{r}, \tilde{\alpha}) \partial_{\tilde{r} \tilde{\alpha}}^{2} \tilde{w}-a_{22}(\tilde{r}, \tilde{\alpha}) \partial_{\tilde{\alpha}}^{2} \tilde{w} \\ & +\tilde{b}_{1}(\tilde{r}, \tilde{\alpha}) \partial_{\tilde{r}} \tilde{w}+b_{2}(\tilde{r}, \tilde{\alpha}) \partial_{\tilde{\alpha}} \tilde{w}- \\ \lim _{\alpha \rightarrow 0+} \tilde{w}=0, & \text { on } \tilde{\alpha}=\alpha_{0}, \\ \tilde{w}=0 & \text { on } \tilde{r}=-x_{1}^{0}, \\ -\partial_{\tilde{r}} \tilde{w}+d_{11}(\tilde{r}, \tilde{\alpha}) \partial_{\tilde{\alpha}} \tilde{w}+\tilde{d}_{10}(\tilde{r}, \tilde{\alpha}) \tilde{w}=0 & \text { on } \tilde{r}=r_{1},\end{cases}
$$

where $\tilde{b}_{0}=b_{0}+O\left(\varepsilon^{1 / 2}+X_{0}^{-1 / 2}\right)$ and $\tilde{d}_{i 0}=d_{i 0}+\left(\varepsilon^{1 / 2}+X_{0}^{-1 / 2}\right)\left(r_{1}+x_{1}^{0}\right)$.

Since $r^{2} \sin ^{2} \alpha \leq r^{2} \sin ^{2} \alpha_{0}<2$ holds for $r \in\left[-x_{1}^{0}-\frac{1}{4}, r_{1}\right]$, then for small $\varepsilon$ and large $X_{0}$ we have $\tilde{b}_{0} \geq 1 / 4$.

In addition, for small $\varepsilon>0$ and large $X_{0}$, we have $\tilde{d}_{i 0} \geq\left(\varepsilon^{1 / 2}+X_{0}^{-1 / 2}\right) / 4$ since $r_{1}+x_{1}^{0} \geq 1$. 
Thus, by the maximum principle for second order elliptic equations (for example, see [Gilbarg and Trudinger 2001, Corollary 3.2 and Theorem 3.1's remark]) we conclude that $\tilde{w} \equiv 0$.

This implies $\partial_{\alpha} V=0$. Hence, $\partial_{\alpha} \varphi_{+} \equiv 0$.

From now on, we will use the notations $\left(\varphi_{+}(r), 0\right)$ instead of $\left(\varphi_{+}(r, \alpha), r(\alpha)\right)$. Then the problem (4-25) can be rewritten as

$$
\begin{cases}c^{2}\left(H_{+}\right) \partial_{r}\left(r^{2} \partial_{r} \varphi_{+}\right)-\frac{r^{2}}{2} \partial_{r} \varphi_{+} \partial_{r}\left(\left|\partial_{r} \varphi_{+}\right|^{2}\right)=0, & \text { on } r=-x_{1}^{0}, \\ \varphi_{+}=\varphi_{-} & \text {on } r=-x_{1}^{0}, \\ \partial_{r} \varphi_{+} H_{+}-\partial_{r} \varphi_{-} H_{-}=0 & \text { on } r=r_{1},\end{cases}
$$

with $H_{+}=H\left(C_{0}-\frac{1}{2}\left(\partial_{r} \varphi_{+}\right)^{2}\right)$.

Since $\frac{1}{2}\left(\partial_{r} \varphi_{+}(r)\right)^{2}+h\left(\rho_{+}(r)\right) \equiv C_{0}$, it follows from the equations above that

$$
\begin{cases}\left(2\left(C_{0}-h\left(\rho_{+}(r)\right)\right)-c^{2}\left(\rho_{+}(r)\right)\right) \partial_{r} \rho_{+}(r) & \\ \quad+\frac{4 \rho_{+}(r)}{r}\left(C_{0}-h\left(\rho_{+}(r)\right)\right)=0 & \text { for }-x_{1}^{0} \leq r \leq\left(1-x_{1}^{0}\right) \sec \alpha_{0} \\ \rho_{+}^{2}(r)\left(C_{0}-h\left(\rho_{+}(r)\right)\right)=\rho_{-}^{2}(r)\left(C_{0}-h\left(\rho_{-}(r)\right)\right) & \text { on } r=-x_{1}^{0}, \\ \rho_{+}(r)=\rho_{+} & \text {on } r=r_{1} .\end{cases}
$$

Let $\rho_{-}\left(-x_{1}^{0}\right)=\rho_{0}$. Then $\rho_{+}\left(-x_{1}^{0}\right)=\rho_{+}$by the Rankine-Hugoniot condition in (4-30). Thus the problem (4-30) can be reduced to

$$
\left\{\begin{array}{l}
\left(2\left(C_{0}-h\left(\rho_{+}(r)\right)\right)-c^{2}\left(\rho_{+}(r)\right)\right) \partial_{r} \rho_{+}(r)+\frac{4 \rho_{+}(r)}{r}\left(C_{0}-h\left(\rho_{+}(r)\right)\right)=0 \\
\rho_{+}\left(-x_{1}^{0}\right)=\rho_{+} \\
\rho_{+}\left(\left(1-x_{1}^{0}\right) \sec \alpha_{0}\right)=\rho_{+}
\end{array}\right.
$$

However, it follows from the first equation in (4-31) that $\partial_{r} \rho_{+}(r)>0$ holds for subsonic flows in the domain $\left\{r:-x_{1}^{0} \leq r \leq r_{1}\right\}$. Hence the problem (4-31) has no solution.

Proof of Theorem 1.5. Let the nozzle be given as in Theorem 1.5. We determine the supersonic incoming flow by solving the following initial-boundary value problem 
for a hyperbolic equation:

$$
\left\{\begin{array}{l}
\sum_{i=1,2,3}\left(\left(\partial_{i} \varphi_{-}\right)^{2}-c_{-}^{2}\right) \partial_{i}^{2} \varphi_{-}+2 \sum_{1 \leq i<j \leq 3} \partial_{i} \varphi_{-} \partial_{j} \varphi_{-} \partial_{i j}^{2} \varphi_{-}=0, \\
\left.\varphi_{-}\right|_{\left(\left(x_{1}-x_{1}^{0}\right)^{2}+x_{2}^{2}+x_{3}^{2}\right)^{1 / 2}=-x_{1}^{0}=0,} \\
\left.\partial_{r} \varphi_{-}\right|_{\left(\left(x_{1}-x_{1}^{0}\right)^{2}+x_{2}^{2}+x_{3}^{2}\right)^{1 / 2}=-x_{1}^{0}}=q_{-}, \\
\partial_{1} f \partial_{1} \varphi_{-}+\sum_{i=2,3}\left(\partial_{i} f-x_{i} / f\right) \partial_{i} \varphi_{-}=0 \quad \text { on } \Pi_{1} \cup \Pi_{2},
\end{array}\right.
$$

where $\left(x_{2}^{2}+x_{3}^{2}\right)^{1 / 2}=f(x)$ represents the nozzle wall $\Pi_{1} \cup \Pi_{2}$.

Then for any given constant pressure $p_{r} \neq p_{r}^{0}$ (here $p_{r}^{0}$ is determined by the first two equations in (4-31)), we have shown by (4-31) that the problem (1-3) with (1-8)-(1-13) has no transonic shock solution $\left(\varphi_{+}(x), \xi\left(x_{2}, x_{3}\right)\right)$ with the regularities and estimates stated in Theorem 1.5.

Remark 4.7. When the assumption (1-8) is removed, it follows from Propositions 4.1-4.5 that the transonic solution is actually symmetric under the assumptions of Theorem 1.5 when the shock lies in the widening part with $-1 / 4 \leq x_{1} \leq 1$. In that case, the potential equations on two sides of the shock $r=r_{0}$ with $-x_{1}^{0}-1 / 4<$ $r_{0}<r_{1}$ can be rewritten $\partial_{r}\left(r^{2} \partial_{r} \varphi_{-}(r) \rho_{-}(r)\right) \equiv 0$ and $\partial_{r}\left(r^{2} \partial_{r} \varphi_{+}(r) \rho_{+}(r)\right) \equiv 0$. By the right hand conditions on the shock in (4-29), we have $r^{2} \partial_{r} \varphi_{+}(r) \rho_{+}(r) \equiv m_{0}$ for $r \geq r_{0}$ with $m_{0}=\left.\left(r^{2} \partial_{r} \varphi_{-}(r) \rho_{-}(r)\right)\right|_{r=-x_{1}^{0}-1 / 4}$. This, together with the Bernoulli's law

$$
\frac{1}{2}\left(\partial_{r} \varphi_{+}(r)\right)^{2}+h\left(\rho_{+}(r)\right) \equiv m_{1} \quad \text { with } m_{1}=\left.\left(\frac{1}{2}\left(\partial_{r} \varphi_{-}(r)\right)^{2}+h\left(\rho_{-}(r)\right)\right)\right|_{r=-x_{1}^{0}-1 / 4}
$$

yields that the end pressure at $r=r_{1}$ is uniquely determined by the supersonic coming flow even if we adjust the position $r_{0}$ of the shock. Thus the transonic shock in a nozzle does not exist for the potential flow equation and for arbitrarily given end pressure. But for the compressible Euler system, this is not the case (since the Bernoulli's constants or entropies on either side of the shock surface are different in the Euler system); see [Xin and Yin 2005b; Xin et al. 2008].

\section{The proof of Theorem 1.8}

In this section, we will prove Theorem 1.8. We use the notations of Section 2. Under the transformation (2-3), it follows from Equation (1-3) and the boundary conditions (1-9), (1-10), (1-11), (1-12)", and (1-13) that the unknown function 
$V(X)$ defined in (2-4) satisfies the following second order equation with the corresponding nonlinear boundary conditions:

$$
\left\{\begin{array}{cl}
\sum_{i, j=1,2,3} a_{i j}\left(X, V, \nabla_{X} V\right) \partial_{X_{i} X_{j}}^{2} V+F_{0}\left(X, V, \nabla_{X} V\right)=0 & \text { in } Q_{+}, \\
G\left(X, V, \nabla_{X} V\right)=0 & \text { on } X_{1}=0, \\
\sum_{j=1,2,3}\left(\sum_{i=2,3}\left(x_{i} / f-\partial_{i} f\right) \partial_{x_{i}} X_{j}-\partial_{1} f \partial_{x_{1}} X_{j}\right) \partial_{X_{j}} V & \\
=\partial_{1} f\left(1-\partial_{1} \varphi_{-}\right)+\sum_{i=2,3}\left(x_{i} / f-\partial_{i} f\right) \partial_{i} \varphi_{-} & \text {on } X_{2}^{2}+X_{3}^{2}=1, \\
\sum_{k=1,2,3} \partial_{x_{1}} X_{k} \partial_{X_{k}} V+\sum_{l=2,3} \sum_{k=1,2,3} b_{l}(x) \partial_{x_{l}} X_{k} \partial_{X_{k}} V+b_{1}(x) V \\
=-1+\partial_{1} \varphi_{-}+b_{2}(x) \partial_{2} \varphi_{-}+b_{3}(x) \partial_{3} \varphi_{-} \\
+b_{1}(x)\left(1-x_{1}+\varphi_{-}(x)\right)-g(x) & \text { on } X_{1}=1 .
\end{array}\right.
$$

We now establish existence, uniqueness and regularity results for problem (5-1).

Theorem 5.1. Let $\delta_{0} \in(0,1 / 3)$ be a given constant. Assume that (1-4)-(1-7) hold. Then there exist positive constants $\varepsilon_{0}$ and $C$ depending only on $\rho_{+}, q_{+}$and $\delta_{0}$ such that for any $\varepsilon \in\left(0, \varepsilon_{0}\right)$, the problem (5-1) has a unique solution $V(X) \in$ $C^{1,1-\delta_{0}}\left(\bar{Q}_{+}\right) \cap C^{3, \delta_{0}}\left(\bar{Q}_{+} \backslash \bigcup_{i=1}^{2} \Gamma_{i}\right)$ with the estimates

$$
\begin{aligned}
& \|V(X)-1\|_{C^{1,1-\delta_{0}}} \leq C \varepsilon, \\
& \left|\nabla_{X}^{k} V(X)\right| \leq C \varepsilon /\left|R_{X}\right|^{k-2+\delta_{0}} \quad \text { for } k=2,3, \\
& \sup _{X, Y \in \bar{Q}_{+} \mid \bigcup_{i=1}^{2}} \sum_{\Gamma_{i}}\left|d_{X, Y}\right|^{1+2 \delta_{0}}\left|\nabla^{k} V(X)-\nabla^{k} V(Y)\right| /|X-Y|^{\delta_{0}} \leq C \varepsilon,
\end{aligned}
$$

and

$$
\begin{aligned}
& \left\|\partial_{\theta} V(X)\right\|_{C^{1,1-\delta_{0}}} \leq C \varepsilon, \\
& \left|\nabla_{X}^{k} \partial_{\theta} V(X)\right| \leq C \varepsilon /\left|R_{X}\right|^{k-2+\delta_{0}} \quad \text { for } k=2,3, \\
& \quad \sup _{X, Y \in \bar{Q}_{+} \mid \bigcup_{i=1}^{2} \Gamma_{i}} \sum_{k=3}\left|d_{X, Y}\right|^{1+2 \delta_{0}}\left|\nabla^{k} \partial_{\theta} V(X)-\nabla^{k} \partial_{\theta} V(Y)\right| /|X-Y|^{\delta_{0}} \leq C \varepsilon,
\end{aligned}
$$

where

$$
\begin{aligned}
\Gamma_{1} & =\left\{\left(0, X_{2}, X_{3}\right): X_{2}^{2}+X_{3}^{2}=1\right\}, \quad \Gamma_{2}=\left\{\left(1, X_{2}, X_{3}\right): X_{2}^{2}+X_{3}^{2}=1\right\}, \\
R_{X} & =X_{1}\left(1-X_{1}\right)+1-\left(X_{2}^{2}+X_{3}^{2}\right), \quad d_{X, Y}=\min \left\{R_{X}, R_{Y}\right\}, \\
\partial_{\theta} & =X_{2} \partial_{3}-X_{3} \partial_{2} .
\end{aligned}
$$


Once Theorem 5.1 is proved, then Theorem 1.8 can be deduced easily from Theorem 5.1 and the generalized partial hodograph transformation (2-3) and (2-4).

The basic strategy for proving Theorem 5.1 is to generalize the proof for the two-dimensional case [Xin and Yin 2005a]. We will use the following Schauder fixed point theorem.

Theorem 5.2 [Gilbarg and Trudinger 2001, Theorem 11.1]. Let $\mathbb{K}$ be a compact, convex subset of a Banach space $\mathbb{B}$, and let $J$ be a continuous mapping from $K$ into itself. Then $J$ has a fixed point in $\mathbb{K}$.

To prove Theorem 5.1, we choose the Banach space $\mathbb{B}$ to be the weighted Hölder space $H_{3, \delta_{0} / 2}^{-\left(2-\tilde{\delta}_{0}\right)}\left(Q_{+}\right)$with constant $\tilde{\delta}_{0} \in\left(\delta_{0}, 1 / 3\right)$, where

$$
\begin{aligned}
& H_{k, \alpha}^{(-m+\beta)}\left(Q_{+}\right) \equiv\left\{W(X) \in C^{m-1,1-\beta}\left(\bar{Q}_{+}\right) \cap C^{k, \alpha}\left(\bar{Q}_{+} \backslash \bigcup_{i=1}^{2} \Gamma_{i}\right)\right. \text { such that } \\
& \|W\|_{C^{m-1,1-\beta}} \leq C ; \\
& \sup _{X}\left|R_{X}\right|^{|l|-m+\beta}\left|\nabla_{X}^{l} W\right| \leq C \quad \text { for } m \leq|l| \leq k \text {; } \\
& \left.\sup _{X, Y \in \bar{Q}_{+} \backslash \bigcup_{i=1}^{2} \Gamma_{i}} \sum_{|l|=k}\left|d_{X, Y}\right|^{k+\alpha-m+\beta} \frac{\left|\nabla^{l} V(X)-\nabla^{l} V(Y)\right|}{|X-Y|^{\alpha}} \leq C\right\}
\end{aligned}
$$

for $k \geq m \geq 1, k, m \in \mathbb{N}$ and $0<\alpha, \beta<1$.

$\mathbb{B}$ is equipped with the norm

$$
\begin{aligned}
\|W\|_{3, \delta_{0} / 2}^{-\left(2-\tilde{\delta}_{0}\right)}=\|W\|_{C^{1,1-\tilde{\delta}_{0}}} & +\sum_{|l|=2,3} \sup _{X}\left|R_{X}\right|^{|l|-2+\tilde{\delta}_{0}}\left|\nabla_{X}^{l} W\right| \\
& +\sup _{X, Y \in \bar{Q}_{+} \backslash \bigcup_{i=1}^{2} \Gamma_{i}} \sum_{|l|=3}\left|d_{X, Y}\right|^{1+\tilde{\delta}_{0}+\delta_{0} / 2} \frac{\left|\nabla^{l} V(X)-\nabla^{l} V(Y)\right|}{|X-Y|^{\delta_{0} / 2}} .
\end{aligned}
$$

It can be shown that $\mathbb{B}$ is a Banach space (since $H_{k, \alpha}^{(-m+\beta)}\left(Q_{+}\right)$is a Banach space with the similar norm; see [Gilbarg and Hörmander 1980; Gilbarg and Trudinger 2001]). The role of $R_{X}$ in $\mathbb{B}$ is to measure the loss of regularity of $W(X)$ near the circles $\Gamma_{1}$ and $\Gamma_{2}$. Sometimes, the subscript $X$ in $R_{X}$ is omitted for convenience.

Next define a subset $\mathbb{K}$ of $\mathbb{B}$ as

$$
\begin{aligned}
& \mathbb{K}=\left\{W(X): W \in H_{3, \delta_{0}}^{-\left(2-\delta_{0}\right)}\left(Q_{+}\right), \partial_{\theta} W\right. \in H_{3, \delta_{0}}^{-\left(2-\delta_{0}\right)}\left(Q_{+}\right), \\
&\left.\|W-1\|_{3, \delta_{0}}^{-\left(2-\delta_{0}\right)} \leq M \varepsilon,\left\|\partial_{\theta} W\right\|_{3, \delta_{0}}^{-\left(2-\delta_{0}\right)} \leq M \varepsilon\right\},
\end{aligned}
$$

where $M \geq 1$ is a constant to be chosen later.

It is clear that $\mathbb{K}$ is a convex subset of $\mathbb{B}$ and that $\mathbb{K}$ is also compact in $\mathbb{B}$; see [Gilbarg and Hörmander 1980]. 
We now define a continuous mapping $J$, which maps $\mathbb{R}$ into itself, by solving an appropriate boundary value problem for some second order linear elliptic equation on a fixed domain with linear boundary conditions; this problem is an appropriate linearization of the nonlinear problem (5-1). More precisely, for any $W \in \mathbb{K}$, we define $J: \mathbb{K} \rightarrow \mathbb{K}$ by

$$
J W=\widetilde{V}+1
$$

where $\widetilde{V}$ is required to solve the equation

$$
\sum_{i, j=1,2,3} a_{i j}\left(X, W, \nabla_{X} W\right) \partial_{X_{i} X_{j}}^{2} \tilde{V}+F_{0}\left(X, W, \nabla_{X} W\right)=0 \quad \text { in } Q_{+} .
$$

Motivated by (2-16), we require $\widetilde{V}$ to satisfy the linear boundary condition on $X_{1}=0$ given by

$$
\sum_{i=1,2,3} B_{1 i}\left(X, W, \nabla_{X} W\right) \partial_{X_{i}} \widetilde{V}+B_{1}\left(X, W, \nabla_{X} W\right)(W-1)=G(X, 1,0,0,0) .
$$

Since $B_{11}\left(X, W, \nabla_{X} W\right) \neq 0$ for small $\varepsilon$, this equation can be rewritten as

$$
\partial_{X_{1}} \widetilde{V}+\sum_{i=2,3} \widetilde{B}_{1 i}\left(X, W, \nabla_{X} W\right) \partial_{X_{i}} \widetilde{V}+\widetilde{B}_{1}\left(X, W, \nabla_{X} W\right)=0 \quad \text { on } X_{1}=0
$$

where the coefficients satisfy

$$
\begin{gathered}
\left\|\widetilde{B}_{1 i}\left(X, W, \nabla_{X} W\right)\right\|_{2, \delta_{0}}^{-\left(1-\delta_{0}\right)}+\left\|\partial_{\theta} \widetilde{B}_{1 i}\left(X, W, \nabla_{X} W\right)\right\|_{2, \delta_{0}}^{-\left(1-\delta_{0}\right)}=O(M \varepsilon), \\
\left\|\widetilde{B}_{1}\left(X, W, \nabla_{X} W\right)\right\|_{2, \delta_{0}}^{-\left(1-\delta_{0}\right)}+\left\|\partial_{\theta} \tilde{B}_{1}\left(X, W, \nabla_{X} W\right)\right\|_{2, \delta_{0}}^{-\left(1-\delta_{0}\right)}=O(\varepsilon),
\end{gathered}
$$

for $i=1,2,3$. This follows from Lemma 2.2. That $\tilde{B}_{1}\left(X, W, \nabla_{X} W\right)=O(\varepsilon)$ will be critical for determining the constant $M$ in $\mathbb{K}$.

Analogously, we require that $\widetilde{V}$ satisfies boundary conditions

$$
\begin{array}{r}
\partial_{X_{1}} \widetilde{V}+\sum_{i=2,3} \widetilde{B}_{2 i}\left(X, W, \nabla_{X} W\right) \partial_{X_{i}} \widetilde{V}+B_{0}(X, W) \widetilde{V}+\widetilde{B}_{2}\left(X, W, \nabla_{X} W\right)=0 \\
\sum_{i=1,2,3} \widetilde{B}_{3 i}\left(X, W, \nabla_{X} W\right) \partial_{X_{i}} \widetilde{V}+\widetilde{B}_{3}\left(X, W, \nabla_{X} W\right)=0
\end{array}
$$

on $X_{1}=1$ and $X_{2}^{2}+X_{3}^{2}=1$, respectively. $\tilde{B}_{2 i}\left(X, W, \nabla_{X} W\right)$ and $\widetilde{B}_{2}\left(X, W, \nabla_{X} W\right)$ have the same estimates as $\widetilde{B}_{1 i}\left(X, W, \nabla_{X} W\right)$ and $\widetilde{B}_{1}\left(X, W, \nabla_{X} W\right)$, respectively, and

$$
\begin{aligned}
& \lambda / 2<B_{0}(X, W)<2 \Lambda, \\
&\left\|B_{0}(X, W)\right\|_{3, \delta_{0}}^{-\left(2-\delta_{0}\right)}+\left\|\partial_{\theta} B_{0}(X, W)\right\|_{3, \delta_{0}}^{-\left(2-\delta_{0}\right)}=O(M \varepsilon) .
\end{aligned}
$$


In addition,

$$
\begin{aligned}
\left\|\widetilde{B}_{31}\left(X, W, \nabla_{X} W\right)\right\|_{2, \delta_{0}}^{-\left(1-\delta_{0}\right)}+\left\|\partial_{\theta} \widetilde{B}_{31}\left(X, W, \nabla_{X} W\right)\right\|_{2, \delta_{0}}^{-\left(1-\delta_{0}\right)} & =O(M \varepsilon), \\
\left\|\widetilde{B}_{3 i}\left(X, W, \nabla_{X} W\right)-X_{i}\right\|_{2, \delta_{0}}^{-\left(1-\delta_{0}\right)} & \\
+\left\|\partial_{\theta}\left(\widetilde{B}_{3 i}\left(X, W, \nabla_{X} W\right)-X_{i}\right)\right\|_{2, \delta_{0}}^{-\left(1-\delta_{0}\right)} & =O(M \varepsilon), \\
\left\|\widetilde{B}_{3}(X, W)\right\|_{3, \delta_{0}}^{-\left(2-\delta_{0}\right)}+\left\|\partial_{\theta} \tilde{B}_{3}(X, W)\right\|_{3, \delta_{0}}^{-\left(2-\delta_{0}\right)} & =O(\varepsilon) .
\end{aligned}
$$

for $i=2,3$.

Since $B_{0}(X, W)>\lambda / 2$, it follows from the maximal principle that

$$
|\widetilde{V}| \leq C_{0} \varepsilon
$$

where the constant $C_{0}>0$ is independent of $M$ and $\varepsilon$.

With the basic $L^{\infty}$ estimate of $\widetilde{V}$ in (5-6), we now can derive the required higher order estimates for $\widetilde{V}$ in order to define the mapping $J$ in (5-2). The desired estimates are stated in the following proposition.

Lemma 5.3. Assume that $W \in \mathbb{K}$. If $\widetilde{V}(X) \in H_{3, \delta_{0}}^{-\left(2-\delta_{0}\right)}\left(Q_{+}\right)$is a solution of (5-3) with the boundary conditions (5-4) and (5-5), then for small $\varepsilon>0$, there exists a constant $C_{0}>0$ independent of $M$ and $\varepsilon$ such that

$$
\|\widetilde{V}\|_{3, \delta_{0}}^{-\left(2-\delta_{0}\right)} \leq C_{0} \varepsilon \quad \text { and } \quad\left\|\partial_{\theta} \tilde{V}\right\|_{3, \delta_{0}}^{-\left(2-\delta_{0}\right)} \leq C_{0} \varepsilon .
$$

Proof. Without loss of generality and for simplicity, we may assume

$$
a_{11}=-1+O(M \varepsilon), \quad a_{22}=-1+O(M \varepsilon), \quad a_{33}=-1+O(M \varepsilon) .
$$

Otherwise, we can use the transformation

$$
X_{1}^{\prime}=X_{1} /\left(c_{+}^{2}-q_{+}^{2}\right)^{1 / 2}, \quad X_{2}^{\prime}=X_{2} / c_{+}, \quad X_{3}^{\prime}=X_{3} / c_{+}
$$

such that the coefficients of the resulting equation satisfy the above requirements.

Set

$$
\begin{aligned}
& \Sigma_{1}=\left\{X: X_{1}=0, X_{2}^{2}+X_{3}^{2}<1\right\}, \quad \Sigma_{2}=\left\{X: 0<X_{1}<1, X_{2}^{2}+X_{3}^{2}=1\right\}, \\
& \Sigma_{3}=\left\{X: X_{1}=1, X_{2}^{2}+X_{3}^{2}<1\right\} .
\end{aligned}
$$

Then $\partial Q_{+}=\overline{\bigcup_{i=1,2,3} \Sigma_{i}}$. Consider a subdomain $Q_{1}$ of $Q_{+}$with the property that $\partial Q_{1} \cap \partial Q_{+}$lies in the interior of $\partial Q_{+}$. Then by the classical Schauder estimates on second order elliptic equations with the uniform oblique derivative boundary conditions (see [Ladyzhenskaya and Ural'tseva 1968; Lieberman 1987]), there exists a constant $C\left(\left\|\widetilde{B}_{k i}\right\|_{C^{1,1-\delta_{0}}\left(\bar{Q}_{1}\right)},\left\|\tilde{B}_{3 j}\right\|_{C^{1,1-\delta_{0}}\left(\bar{Q}_{1}\right)}\right)$ for $k=1,2, i=2,3$, and $j=1,2,3$ such that

(5-7) $\|\tilde{V}\|_{C^{2,1-\delta_{0}}\left(\bar{Q}_{1}\right)} \leq C\left(\|\tilde{V}\|_{L^{\infty}\left(Q_{+}\right)}+\left\|F_{0}\right\|_{C^{1-\delta_{0}}\left(Q_{+}\right)}+\sum_{i=1,2,3}\left\|\tilde{B}_{i}\right\|_{C^{1,1-\delta_{0}}\left(Q_{+}\right)}\right)$. 
Thus our main task is to estimate the derivatives of $\widetilde{V}$ near the circles $\Gamma_{1}$ and $\Gamma_{2}$. To this end, without loss of generality we consider only the problem in a small neighborhood $G\left(r_{0}\right)=\left\{X:\left|X-P_{0}\right|<r_{0}\right\}$ of $P_{0}=(0,1,0)$.

We will use the cylindrical coordinates (3-11) and let $Z_{1}=X_{1}, Z_{2}=r$, and $Z_{3}=\theta$. Then in the domain

$$
G^{\prime}\left(r_{0}\right)=G\left(r_{0}\right) \cap\left\{Z: Z_{1} \geq 0,1-\delta \leq Z_{2} \leq 1,-r_{0} \leq Z_{3} \leq r_{0}\right\},
$$

with a constant $\delta \in(0,1)$, Equation (5-3) and boundary conditions (5-4) and (5-5) can be rewritten as

$$
\begin{cases}\sum_{i, j=1,2,3} A_{i j}(Z) \partial_{Z_{i} Z_{j}}^{2} \tilde{V}+\sum_{i=1,2,3} M_{i}(Z) \partial_{Z_{i}} \tilde{V}=F(Z) \\ \partial_{Z_{1}} \widetilde{V}+\sum_{i=2,3} N_{1 i}(Z) \partial_{Z_{i}} \tilde{V}=G_{1}(Z) & \text { on } Z_{1}=0, \\ \partial_{Z_{2}} \tilde{V}+N_{21}(Z) \partial_{Z_{1}} \tilde{V}+N_{23}(Z) \partial_{Z_{3}} \tilde{V}=G_{2}(Z) & \text { on } Z_{2}=1,\end{cases}
$$

where

$$
\begin{gathered}
\sum_{i=1,2,3}\left\|M_{i}(Z)\right\|_{2, \delta_{0}}^{-\left(1-\delta_{0}\right)} \leq C(1+M \varepsilon), \\
\sum_{i, j=1,2,3}\left\|A_{i j}(Z)+\delta_{i j}\right\|_{2, \delta_{0}}^{-\left(1-\delta_{0}\right)} \leq C\left(r_{0}+M \varepsilon\right), \\
\sum_{i=1,2}\left\|G_{i}(Z)\right\|_{2, \delta_{0}}^{-\left(1-\delta_{0}\right)}+\|F(Z)\|_{2, \delta_{0}}^{-\left(1-\delta_{0}\right)} \leq C \varepsilon, \\
\left\|N_{12}(Z)\right\|_{2, \delta_{0}}^{-\left(1-\delta_{0}\right)}+\left\|N_{13}(Z)\right\|_{2, \delta_{0}}^{-\left(1-\delta_{0}\right)}+\left\|N_{21}(Z)\right\|_{2, \delta_{0}}^{-\left(1-\delta_{0}\right)}+\left\|N_{23}(Z)\right\|_{2, \delta_{0}}^{-\left(1-\delta_{0}\right)} \leq C M \varepsilon,
\end{gathered}
$$

with the weight $R_{Z}=\left(Z_{1}^{2}+\left(Z_{2}-1\right)^{2}\right)^{1 / 2}$ and $d_{Z, Z^{\prime}}=\min \left(R_{Z}, R_{Z^{\prime}}\right)$. The generic constant $C>0$ is independent of $M, \varepsilon$, and $r_{0}$.

Define a $C^{\infty}$ function $\chi(Z)$ such that

$$
\chi(Z)= \begin{cases}1 & \text { if }\left(\left|Z_{1}\right|^{2}+\left|Z_{2}-1\right|^{2}+\left|Z_{3}\right|^{2}\right)^{1 / 2} \leq r_{0} / 2, \\ 0 & \text { if }\left(\left|Z_{1}\right|^{2}+\left|Z_{2}-1\right|^{2}+\left|Z_{3}\right|^{2}\right)^{1 / 2} \geq(2 / 3) r_{0} .\end{cases}
$$

Let $\widetilde{V}_{1}(Z)=\chi(Z) \widetilde{V}$. Then it follows from $(5-8)$ that $V_{1}(Z)$ satisfies the elliptic equation with boundary conditions given by

$$
\sum_{i, j=1,2,3} A_{i j}(Z) \partial_{Z_{i} Z_{j}}^{2} \tilde{V}_{1}+\sum_{i=1,2,3} M_{i}(Z) \partial_{Z_{i}} \tilde{V}_{1}=F^{\prime}(Z),
$$

$$
\begin{array}{lr}
\partial_{Z_{1}} \widetilde{V}_{1}+\sum_{i=2,3} N_{1 i}(Z) \partial_{Z_{i}} \tilde{V}_{1}=G_{1}^{\prime}(Z) & \text { on } Z_{1}=0, \\
\partial_{Z_{2}} \widetilde{V}_{1}+N_{21}(Z) \partial_{Z_{1}} \widetilde{V}_{1}+N_{23}(Z) \partial_{Z_{3}} \tilde{V}_{1}=G_{2}^{\prime}(Z) & \text { on } Z_{2}=1, \\
\widetilde{V}_{1}=0 & \text { on }\left(\left|Z_{1}\right|^{2}+\left|Z_{2}-1\right|^{2}+\left|Z_{3}\right|^{2}\right)^{1 / 2}=r_{0} .
\end{array}
$$


Combining this with the Schauder interior estimate (5-7), we derive easily that $F^{\prime}(Z)$ and $G_{i}^{\prime}(Z)$ have the same properties as $F(Z)$ and $G_{i}(Z)$.

Now, by choosing $\delta=1-\delta_{0}$ and $\alpha=1-\delta_{0}$ in [Lieberman 1988, 3.4] and noting that the angle between $Z_{1}=0$ and $Z_{1}=1$ is $\pi / 2$, we can check carefully the proof of [Lieberman 1988, Lemma 3.1] to verify that its conclusions still hold in this case; see [Xin and Yin 2005a] for details. Using this and (5-6), we find that for small $r_{0}$ and $M \varepsilon$, we have $\left\|\widetilde{V}_{1}\right\|_{2,1-\delta_{0}}^{-\left(2-\delta_{0}\right)} \leq C_{0} \varepsilon$, where $C_{0}$ is a uniform generic constant.

Moreover, by using an argument similar to that of [Gilbarg and Trudinger 2001, Theorem 6.17] for the higher regularities of solutions to the second order elliptic equations there, it follows from [Lieberman 1988, Lemma 3.1] that

$$
\left\|\widetilde{V}_{1}\right\|_{3, \delta_{0}}^{-\left(2-\delta_{0}\right)} \leq C_{0} \varepsilon
$$

Next we improve the estimates of the tangential derivatives of $\tilde{V}_{1}$.

Set $U=\partial_{3} \widetilde{V}_{1}$. It follows from (5-9), (5-10) and the assumption on the tangent regularities of $W(X)$ that

$$
\begin{array}{lr}
\sum_{i, j=1,2,3} A_{i j}(Z) \partial_{Z_{i} Z_{j}}^{2} U+\sum_{i=1,2,3} M_{i}(Z) \partial_{Z_{i}} U=F_{1}(Z), \\
\partial_{Z_{1}} U+\sum_{i=2,3} N_{1 i}(Z) \partial_{Z_{i}} U=H_{1}(Z) & \text { on } Z_{1}=0, \\
\partial_{Z_{2}} U+N_{21}(Z) \partial_{Z_{1}} U+N_{23}(Z) \partial_{Z_{3}} U=H_{2}(Z) & \text { on } Z_{2}=1, \\
U=0 \quad \text { on }\left(\left|Z_{1}\right|^{2}+\left|Z_{2}-1\right|^{2}+\left|Z_{3}\right|^{2}\right)^{1 / 2}=r_{0},
\end{array}
$$

where

$$
\sum_{i=1,2}\left\|H_{i}(Z)\right\|_{2, \delta_{0}}^{-\left(1-\delta_{0}\right)}+\left\|F_{1}(Z)\right\|_{2, \delta_{0}}^{-\left(1-\delta_{0}\right)} \leq C \varepsilon .
$$

Analogously, we can obtain $\|U\|_{3, \delta_{0}}^{-\left(2-\delta_{0}\right)} \leq C \varepsilon$. Combining this with (5-10) shows that Lemma 5.3 holds.

Due to (5-6) and Lemma 5.3 and by the continuity method given in [Gilbarg and Trudinger 2001] (see also [Nazarov and Plamenevsky 1994] or [Lieberman 1988, Lemma 2.3]), the linear Equation (5-3) with the boundary conditions (5-4) and (5-5) is solvable in the space $\mathbb{K}$. Furthermore, (5-6) and Lemma 5.3 imply that we can choose the constant $C_{0}$ as the constant $M$ in $\mathbb{K}$. Hence the mapping $J$, which is defined in (5-2), maps from $\mathbb{K}$ into $\mathbb{K}$. Moreover, by the maximal principle and the estimates in Lemma 5.3, $J$ is a continuous mapping from $\mathbb{K} \rightarrow \mathbb{K}$. We are now ready to complete the proof of Theorem 5.1:

Proof of Theorem 5.1. It follows from Lemma 5.3 that the mapping $J$ satisfies the all requirements of Theorem 5.2. By the choice of $J$, the existence of a solution 
in Theorem 5.1 follows. In addition, the maximal principle in [Lieberman 1987] implies the uniqueness in Theorem 5.1. This completes the proof.

Proof of Theorem 1.8. Part (i) of Theorem 1.8 follows directly from Lemma 2.1.

By the regularity and uniqueness of $V(X)$ in Theorem 5.1, one concludes that the inverse transformation (2-3) has the properties:

$$
x_{1}(X), x_{2}(X), x_{3}(X) \in C^{1,1-\delta_{0}}\left(\bar{Q}_{+}\right) \cap C^{3, \delta_{0}}\left(Q_{+}\right) .
$$

Since the shock $\Sigma$ : $x_{1}=\xi\left(x_{2}, x_{3}\right)$ corresponds to $X_{1}=0$ in $\bar{Q}_{+}$, we have $\xi\left(x_{2}, x_{3}\right) \in C^{1,1-\delta_{0}}(\bar{S}) \cap C^{3, \delta_{0}}(S)$, where $S$ represents the open projection set of $\Sigma$ onto the $\left(x_{2}, x_{3}\right)$-plane. Also, by using the properties of $V(X)$ in Theorem 5.1, we easily verify the remaining conclusions (ii), (iii), and (iv) in Theorem 1.8. This complete the proof.

\section{Appendix}

In this appendix, we will give some details on the properties of the transonic shock problem after the generalized hodograph transformation in Section 2 and some exact formulas used in the proofs of Proposition 4.1. In particular, we will prove Lemma 2.2 and Lemma 2.4.

Proof of Lemma 2.2. We start with the proof of (2-17). By definition, we have

$$
\begin{aligned}
G(X, 1,0,0,0)=\left(H\left(C_{0}-\frac{1}{2}\left(\left(1-\partial_{1} \varphi_{-}\right)^{2}+\sum_{i=2,3}\left(\partial_{i} \varphi_{-}\right)^{2}\right)\right)\left(\partial_{1} \varphi_{-}-1\right)\right. \\
\left.-H\left(C_{0}-\frac{1}{2}\left|\nabla \varphi_{-}\right|^{2}\right) \partial_{1} \varphi_{-}\right)(\bar{x})
\end{aligned}
$$

with $\bar{x}=\left(\bar{x}_{1}, \bar{x}_{2}, \bar{x}_{3}\right)$ given by $\bar{x}_{1}=X_{1}$ and $\bar{x}_{i}=\bar{x}_{i}\left(X_{1}, X_{2}, X_{3}\right)$ for $i=2,3$.

More precisely, $\left(\bar{x}_{2}, \bar{x}_{3}\right)$ is determined by

$$
X_{2}=\bar{x}_{2}(f(\bar{x}))^{-1} \quad \text { and } \quad X_{3}=\bar{x}_{3}(f(\bar{x}))^{-1} .
$$

Using Lemma 2.1 and the assumption that $q_{-}-q_{+}=1$, we compute that

$$
\begin{aligned}
G(X, 1,0,0,0)= & \left(H\left(C_{0}-\frac{1}{2}\left(q_{-}-1\right)^{2}+O(\varepsilon)\right)\left(q_{-}-1+O(\varepsilon)\right)\right. \\
& \left.-H\left(C_{0}-\frac{1}{2} q_{-}^{2}+O(\varepsilon)\right)\left(q_{-}+O(\varepsilon)\right)\right)(\bar{x}) \\
= & \left(H\left(C_{0}-\frac{1}{2}\left(q_{-}-1\right)^{2}\right)\left(q_{-}-1\right)-H\left(C_{0}-\frac{1}{2} q_{-}^{2}\right) q_{-}\right)+O(\varepsilon) \\
= & \left(\rho_{+} q_{+}-\rho_{0} q_{-}\right)+O(\varepsilon)=O(\varepsilon),
\end{aligned}
$$

where we have used $\rho_{+} q_{+}=\rho_{0} q_{-}$; see (1-7). Thus $|G(X, 1,0,0,0)| \leq C \varepsilon$. Similarly, noting also (1-4), we have

$$
\sum_{k=1,2,3}\left|\nabla_{X}^{k} G(X, 1,0,0,0)\right| \leq C \varepsilon
$$


which proves (2-17).

Next, we verify the rest of the lemma, namely, (2-18)-(2-21). Direct calculations based on (2-5) and (2-6) show that

(A.1)

$$
\begin{aligned}
\frac{\partial x_{1}}{\partial V} & =X_{1}-1, \\
\frac{\partial x_{k}}{\partial V} & =\left(X_{1}-1\right) \partial_{X_{1}} f\left(1-\left(X_{2} \partial_{x_{2}} f+X_{3} \partial_{x_{3}} f\right)\right)^{-1} X_{k}, \\
\frac{\partial\left(\partial_{x_{i}} V\right)}{\partial\left(\partial_{X_{j}} V\right)} & =D\left(X, V, \partial_{X} V\right) V \frac{\partial X_{j}}{\partial x_{i}}, \\
\frac{\partial\left(\partial_{i} \varphi_{-}\right)}{\partial V} & =\sum_{j=1,2,3} \partial_{i j}^{2} \varphi_{-}(x) \frac{\partial x_{j}}{\partial V}, \\
\frac{\partial\left(\partial_{i} \varphi_{+}\right)}{\partial V} & =\sum_{j=1,2,3}\left(\partial_{i j}^{2} \varphi_{-}(x) \frac{\partial x_{j}}{\partial V}-\partial_{X_{j}} V \frac{\partial}{\partial V}\left(\frac{\partial X_{j}}{\partial x_{i}}\right)\right),
\end{aligned}
$$

for $k=2,3$, and $i, j=1,2,3$. Define

$$
\bar{G}\left(\nabla \varphi_{+}, \nabla \varphi_{-}\right)=\sum_{j=1,2,3}\left[\partial_{j} \varphi H\right] \partial_{j}\left(\varphi_{+}-\varphi_{-}\right) .
$$

Then $G\left(X, V, \nabla_{X} V\right)=\bar{G}\left(\nabla \varphi_{+}, \nabla \varphi_{-}\right)$, and

$$
\begin{aligned}
\partial_{\partial_{X_{i}} V} G & =-V D\left(X, V, \nabla_{X} V\right) \sum_{k=1,2,3} \partial_{\partial_{k} \varphi_{+}} \bar{G} \frac{\partial X_{i}}{\partial x_{k}}, \\
\partial_{V} G & =\sum_{k=1,2,3}\left(\partial_{\partial_{k} \varphi_{+}} \bar{G} \frac{\partial\left(\partial_{k} \varphi_{+}\right)}{\partial V}+\partial_{\partial_{k} \varphi_{-}} \bar{G} \frac{\partial\left(\partial_{k} \varphi_{-}\right)}{\partial V}\right), \\
\partial_{\partial_{i} \varphi_{+}} \bar{G} & =\sum_{j=1,2,3}\left(\left[\partial_{j} \varphi H\right] \delta_{i j}+\left(H_{+} \delta_{i j}-\partial_{j} \varphi_{+} \partial_{i} \varphi_{+} H_{+}^{\prime}\right)\left(\partial_{j} \varphi_{+}-\partial_{j} \varphi_{-}\right)\right), \\
\partial_{\partial_{i} \varphi_{-}} \bar{G} & =-\sum_{j=1,2,3}\left(\left[\partial_{j} \varphi H\right] \delta_{i j}+\left(H_{-} \delta_{i j}-\partial_{j} \varphi_{-} \partial_{i} \varphi_{-} H_{-}^{\prime}\right)\left(\partial_{j} \varphi_{+}-\partial_{j} \varphi_{-}\right)\right),
\end{aligned}
$$

for $i=1,2,3$. We can obtain from (2-6) and (2-15) that

$$
\partial_{x_{j}} X_{i}=\delta_{i j}+O(\varepsilon) \text { for } i, j=1,2,3,
$$

and it follows from (A.1), (A.3), and Lemma 2.1 that

$$
\begin{aligned}
& \left(\frac{\partial\left(\partial_{i} \varphi_{ \pm}\right)}{\partial V}\right)(X, V)=O(\varepsilon), \quad \text { for } i=1,2,3, \\
& \left(\frac{\partial\left(\partial_{x_{i}} V\right)}{\partial\left(\partial_{X_{j}} V\right)}\right)\left(X, V, \nabla_{X} V\right)=\delta_{i j}+O(\varepsilon) \quad \text { for } i, j=1,2,3 \text {. }
\end{aligned}
$$


Note that

$$
\partial_{\partial_{i} \varphi_{ \pm}} \bar{G}= \begin{cases} \pm \frac{\rho_{+}\left(q_{+}-q_{-}\right)\left(c^{2}\left(\rho_{+}\right)-q_{+}^{2}\right)}{c^{2}\left(\rho_{+}\right)}(1+O(\varepsilon)) & \text { if } i=1, \\ O(\varepsilon) & \text { if } i=2,3 .\end{cases}
$$

Indeed, recall that we have assumed that $q_{-}-q_{+}=1$; then it follows from $V(X)=1-x_{1}+\varphi_{-}(x)-\varphi_{+}(x)$ that $\partial_{i} \varphi_{+}=\partial_{i} \varphi_{-}-\delta_{1 i}-\nabla_{X} V \partial X / \partial x_{i}$. So one can derive from Lemma 2.1 and (A.3) that

$$
\partial_{i} \varphi_{ \pm}= \begin{cases}q_{ \pm}+O(\varepsilon) & \text { if } i=1, \\ O(\varepsilon) & \text { if } i=2,3 .\end{cases}
$$

On the other hand, the Bernoulli's law, (1-1), and (1-2) imply that $c^{2}(\rho)=H / H^{\prime}$. Hence, we obtain from (A.2) and (A.5) that

$$
\begin{aligned}
\partial_{\partial_{1} \varphi_{+}} \bar{G} & =\left[\partial_{1} \varphi H\right]+\left(H_{+}\left(\partial_{1} \varphi_{+}-\partial_{1} \varphi_{-}\right)-\sum_{j=1,2,3}\left(\partial_{j} \varphi_{+} \partial_{1} \varphi_{+} H_{+}^{\prime}\right)\left(\partial_{j} \varphi_{+}-\partial_{j} \varphi_{-}\right)\right) \\
& =\left(\partial_{1} \varphi_{+} H_{+}-\partial_{1} \varphi_{-} H_{-}\right)+H_{+}\left(\partial_{1} \varphi_{+}-\partial_{1} \varphi_{-}\right)\left(1-\left(\partial_{1} \varphi_{+}\right)^{2} \frac{H_{+}^{\prime}}{H_{+}}\right)+O(\varepsilon) \\
& =\frac{\rho_{+}\left(q_{+}-q_{-}\right)\left(c^{2}\left(\rho_{+}\right)-q_{+}^{2}\right)}{c^{2}\left(\rho_{+}\right)}(1+O(\varepsilon)) .
\end{aligned}
$$

The other estimates in (A.4) can be obtained similarly.

Substituting the computations above into expressions for $B_{1 i}$ and $B_{1}$ yields

$$
\begin{aligned}
& B_{1 i}\left(X, V, \nabla_{X} V\right)= \begin{cases}-\frac{\rho_{+}\left(q_{+}-q_{-}\right)\left(c^{2}\left(\rho_{+}\right)-q_{+}^{2}\right)}{c^{2}\left(\rho_{+}\right)}(1+O(\varepsilon)) & \text { if } i=1, \\
O(\varepsilon) & \text { if } i=2,3,\end{cases} \\
& B_{1}\left(X, V, \nabla_{X} V\right)(W-1)=O(\varepsilon) .
\end{aligned}
$$

These prove (2-18)-(2-20). The other property (2-21) can be verified similarly. Hence the proof of Lemma 2.2 is completed.

Next, we sketch the proof of Lemma 2.4.

Proof of Lemma 2.4. The proof will be sketched since it involves mostly tedious computations. By (2-10), we have

$$
\begin{aligned}
a_{11}\left(X, V, \nabla_{X} V\right)=\sum_{k=1,2,3}\left(\left(\partial_{k} \varphi_{+}\right)^{2}-c_{+}^{2}\right) & \left(\left(\partial_{x_{k}} X_{1}\right)^{2}+b_{11}^{k k} \partial_{X_{1}} V\right) \\
& +\sum_{k \neq l} \partial_{k} \varphi_{+} \partial_{l} \varphi_{+}\left(\partial_{x_{k}} X_{1} \partial_{x_{l}} X_{1}+b_{11}^{k l} \partial_{X_{1}} V\right) .
\end{aligned}
$$

Taking into account (A.3), (A.5), (2-6) and (2-15), we can get from the above that

$$
a_{11}\left(X, V, \nabla_{X} V\right)=\left(q_{+}^{2}-c_{+}^{2}\right)(1+O(\varepsilon)) .
$$


Similarly, for $i=2,3$,

$$
\begin{aligned}
a_{i i}\left(X, V, \nabla_{X} V\right)= & \sum_{k=1,2,3}\left(\left(\partial_{k} \varphi_{+}\right)^{2}-c_{+}^{2}\right)\left(\left(\partial_{x_{k}} X_{i}\right)^{2}+b_{i i}^{k k} \partial_{X_{1}} V\right) \\
& +\sum_{k \neq l} \partial_{k} \varphi_{+} \partial_{l} \varphi_{+}\left(\partial_{x_{k}} X_{i} \partial_{x_{l}} X_{i}+b_{i i}^{k l} \partial_{X_{1}} V\right) \\
=-c_{+}^{2}\left(\partial_{x_{i}} X_{i}\right)^{2}+ & O(\varepsilon)=-c_{+}^{2}(1+O(\varepsilon)),
\end{aligned}
$$

and, for $i \neq j$,

$$
\begin{aligned}
a_{i j}\left(X, V, \nabla_{X} V\right)=\sum_{k=1,2,3}\left(\left(\partial_{k} \varphi_{+}\right)^{2}-c_{+}^{2}\right)\left(\partial_{x_{k}} X_{i} \partial_{x_{k}} X_{j}+b_{i j}^{k k} \partial_{X_{1}} V\right) \\
\quad+\sum_{k \neq l} \partial_{k} \varphi_{+} \partial_{l} \varphi_{+}\left(\partial_{x_{k}} X_{i} \partial_{x_{l}} X_{j}+b_{i j}^{k l} \partial_{X_{1}} V\right)=O(\varepsilon) .
\end{aligned}
$$

Next, we derive the estimate $\left|b_{0}^{l k}\left(X, V, \nabla_{X} V\right)\right|=O(\varepsilon)$ from (2-6)-(2-8), (A.3), and (2-15). This with (2-10) and Lemma 2.1 leads to $\left|F_{0}\left(X, V, \nabla_{X} V\right)\right|=O(\varepsilon)$.

The rest of Lemma 2.4 follows from similar arguments and direct computations. This proves Lemma 2.4 .

Next, we provide the explicit expressions of the coefficients for the second order equation in (4-14) and (4-15) in the proof of Proposition 4.1.

(A.6)

$$
\begin{aligned}
& a_{11}(\tilde{r}, \tilde{\theta}, \tilde{\alpha})=-\left(1-\frac{\left(\partial_{r} \varphi_{+}\right)^{2}}{c^{2}\left(H_{+}\right)}\right)\left(\left(\tilde{r}-r_{1}\right) A_{0}^{2} \partial_{\tilde{r}} V-A_{0}\right)\left(r_{1}+x_{1}^{0}\right) \partial_{r} \tilde{r} \\
& \quad+\frac{2\left(\tilde{r}-r_{1}\right)}{r^{2} c^{2}\left(H_{+}\right)} \partial_{r} \varphi_{+} \partial_{\alpha} \varphi_{+} A_{0}^{2} V \partial_{\tilde{\alpha}} V \partial_{r} \tilde{r}-\frac{\tilde{r}-r_{1}}{r^{2}}\left(1-\frac{\left(\partial_{\alpha} \varphi_{+}\right)^{2}}{r^{2} c^{2}\left(H_{+}\right)}\right) A_{0}^{2} V \partial_{\tilde{\alpha}} V \partial_{\alpha} \tilde{r} \\
& \quad-\frac{\tilde{r}-r_{1}}{r^{2} \sin ^{2} \alpha}\left(1-\frac{\left(\partial_{\theta} \varphi_{+}\right)^{2}}{r^{2} \sin ^{2} \alpha c^{2}\left(H_{+}\right)}\right) A_{0}^{2} V \partial_{\tilde{\theta}} V \partial_{\theta} \tilde{r} \\
& +\frac{2 \partial_{r} \varphi_{+} \partial_{\theta} \varphi_{+}}{r^{2} \sin ^{2} \alpha c^{2}\left(H_{+}\right)}\left(\tilde{r}-r_{1}\right) A_{0}^{2} V \partial_{\tilde{\theta}} V \partial_{r} \tilde{r}+\frac{2 \partial_{\alpha} \varphi_{+} \partial_{\theta} \varphi_{+}}{r^{4} \sin ^{2} \alpha c^{2}\left(H_{+}\right)}\left(\tilde{r}-r_{1}\right) A_{0}^{2} V \partial_{\tilde{\theta}} V \partial_{\alpha} \tilde{r} \\
& +\frac{1}{r^{2} \sin ^{2} \alpha}\left(1-\frac{\left(\partial_{\theta} \varphi_{+}\right)^{2}}{r^{2} \sin ^{2} \alpha c^{2}\left(H_{+}\right)}\right) A_{0} V p_{3}\left(A_{0} \partial_{\tilde{\theta}} V\left(\tilde{r}-r_{1}\right)+p_{3}-\partial_{\theta} \tilde{r}\right) \\
& \quad+\frac{2 \partial_{r} \varphi_{+} \partial_{\theta} \varphi_{+}}{r^{2} \sin ^{2} \alpha c^{2}\left(H_{+}\right)} A_{0} V p_{3} \partial_{r} \tilde{r}+\frac{2 \partial_{\alpha} \varphi_{+} \partial_{\theta} \varphi_{+}}{r^{4} \sin ^{2} \alpha c^{2}\left(H_{+}\right)} A_{0} V p_{3} \partial_{\alpha} \tilde{r} .
\end{aligned}
$$

$$
\begin{aligned}
a_{12}(\tilde{r}, \tilde{\theta}, \tilde{\alpha}) & =-\frac{1}{r^{2} c^{2}\left(H_{+}\right)} \partial_{r} \varphi_{+} \partial_{\alpha} \varphi_{+} A_{0} V \partial_{r} \tilde{r} \\
& -\frac{1}{2 r^{2}}\left(1-\frac{\left(\partial_{\alpha} \varphi_{+}\right)^{2}}{r^{2} c^{2}\left(H_{+}\right)}\right)\left(\left(\tilde{r}-r_{1}\right) A_{0}^{2} V \partial_{\tilde{\alpha}} V-A_{0} V \partial_{\alpha} \tilde{r}\right) \\
& +\frac{\partial_{\alpha} \varphi_{+} \partial_{\theta} \varphi_{+}}{r^{4} \sin ^{2} \alpha c^{2}\left(H_{+}\right)}\left(\tilde{r}-r_{1}\right) A_{0}^{2} V \partial_{\tilde{\theta}} V+\frac{2 \partial_{\alpha} \varphi_{+} \partial_{\theta} \varphi_{+}}{r^{4} \sin ^{2} \alpha c^{2}\left(H_{+}\right)} A_{0} V p_{3} .
\end{aligned}
$$

$a_{22}(\tilde{r}, \tilde{\theta}, \tilde{\alpha})=\frac{1}{r^{2}}\left(1-\frac{\left(\partial_{\alpha} \varphi_{+}\right)^{2}}{r^{2} c^{2}\left(H_{+}\right)}\right) A_{0} V$, 
(A.7) $f(\tilde{r}, \tilde{\theta}, \tilde{\alpha})=\frac{\partial_{\alpha} \varphi_{+}}{r^{2} \operatorname{tg} \alpha}+\frac{2}{r} \partial_{r} \varphi_{+}$

$$
\begin{gathered}
\quad \frac{\partial_{r} \varphi_{+}\left(\partial_{\alpha} \varphi_{+}\right)^{2}}{r^{3} c^{2}\left(H_{+}\right)}+\left(1-\frac{\left(\partial_{r} \varphi_{+}\right)^{2}}{c^{2}\left(H_{+}\right)}\right)\left(\varphi_{-}^{\prime \prime}(r)+2 A_{0}^{2}\left(r_{1}+x_{1}^{0}\right)\left(\partial_{\tilde{r}} V\right)^{2} \partial_{r} \tilde{r}\right) \\
+\frac{2}{r^{2} c^{2}\left(H_{+}\right)} \partial_{r} \varphi_{+} \partial_{\alpha} \varphi_{+}\left(A_{0} \partial_{\tilde{r}} V \partial_{\tilde{\alpha}} V-2 A_{0}^{2} V \partial_{\tilde{r}} V \partial_{\tilde{\alpha}} V\right) \partial_{r} \tilde{r} \\
-\frac{1}{r^{2}}\left(1-\frac{\left(\partial_{\alpha} \varphi_{+}\right)^{2}}{r^{2} c^{2}\left(H_{+}\right)}\right)\left(\partial_{\tilde{\alpha}} V-A_{0} V \partial_{\tilde{\alpha}} V+\partial_{\tilde{r}} V \partial_{\alpha} \tilde{r}-2 A_{0} V \partial_{\tilde{r}} V \partial_{\alpha} \tilde{r}\right) A_{0} \partial_{\tilde{\alpha}} V \\
-\frac{1}{r^{2} \sin ^{2} \alpha}\left(1-\frac{\left(\partial_{\theta} \varphi_{+}\right)^{2}}{r^{2} \sin ^{2} \alpha c^{2}\left(H_{+}\right)}\right)\left(A_{0}\left(\partial_{\tilde{\theta}} V\right)^{2}-A_{0}^{2} V\left(\partial_{\tilde{\theta}} V\right)^{2}-A_{0}^{2}\left(\tilde{r}-r_{1}\right) V \partial_{\tilde{\theta}} V p_{5}\right. \\
\left.+A_{0} V p_{4}+A_{0} \partial_{\tilde{r}} V \partial_{\tilde{\theta}} V \partial_{\theta} \tilde{r}+A_{0} V p_{5} \partial_{\theta} \tilde{r}-2 A_{0}^{2} V \partial_{\tilde{\theta}} V \partial_{\tilde{r}} V \partial_{\theta} \tilde{r}\right) \\
+\frac{2 \partial_{r} \varphi_{+} \partial_{\theta} \varphi_{+}}{r^{2} \sin ^{2} \alpha c^{2}\left(H_{+}\right)}\left(A_{0}\left(\partial_{\tilde{r}} V \partial_{\tilde{\theta}} V+V p_{5}\right)-2 A_{0}^{2} V \partial_{\tilde{\theta}} V \partial_{\tilde{r}} V\right) \partial_{r} \tilde{r} \\
+\frac{2 \partial_{\alpha} \varphi_{+} \partial_{\theta} \varphi_{+}}{r^{4} \sin ^{2} \alpha c^{2}\left(H_{+}\right)}\left(A_{0}\left(\partial_{\tilde{r}} V \partial_{\tilde{\theta}} V+V p_{5}\right) \partial_{\alpha} \tilde{r}+A_{0}\left(\partial_{\tilde{\alpha}} V \partial_{\tilde{\theta}} V+V p_{6}\right)\right. \\
+\frac{\partial_{r} \varphi_{+}\left(\partial_{\theta} \varphi_{+}\right)^{2}}{r^{3} \sin ^{2} \alpha c^{2}\left(H_{+}\right)}+\frac{\partial_{\alpha} \varphi_{+}\left(\partial_{\theta} \varphi_{+}\right)^{2} \cos \alpha}{r^{4} \sin ^{3} \alpha c^{2}\left(H_{+}\right)} .
\end{gathered}
$$

\section{Acknowledgments}

The authors would like to thank Professor C. Morawetz, who read the paper and offered many comments and suggestions to improve the paper.

\section{References}

[Alt et al. 1985] H. W. Alt, L. A. Caffarelli, and A. Friedman, "Compressible flows of jets and cavities”, J. Differential Equations 56:1 (1985), 82-141. MR 86i:35036 Zbl 0614.76074

[Bers 1954] L. Bers, "Existence and uniqueness of a subsonic flow past a given profile", Comm. Pure Appl. Math. 7 (1954), 441-504. MR 16,417a Zbl 0058.40601

[Čanić et al. 2000] S. Čanić, B. L. Keyfitz, and G. M. Lieberman, "A proof of existence of perturbed steady transonic shocks via a free boundary problem", Comm. Pure Appl. Math. 53:4 (2000), 484511. MR 2001m:76056 Zbl 1017.76040

[Chen and Feldman 2003] G.-Q. Chen and M. Feldman, "Multidimensional transonic shocks and free boundary problems for nonlinear equations of mixed type", J. Amer. Math. Soc. 16:3 (2003), 461-494. MR 2004d:35182 Zbl 1015.35075

[Chen et al. 2002] S. Chen, Z. Xin, and H. Yin, "Unsteady supersonic flow past a wedge", Preprint, Institute of Mathematical Sciences at The Chinese University of Hong Kong, 2002.

[Courant and Friedrichs 1948] R. Courant and K. O. Friedrichs, Supersonic Flow and Shock Waves, Interscience Publishers, New York, 1948. MR 10,637c Zbl 0041.11302 
[Fletcher 1991a] C. A. J. Fletcher, Computational techniques for fluid dynamics, I: Fundamental and general techniques, Second ed., Springer, Berlin, 1991. MR 92e:76042 Zbl 0717.76001

[Fletcher 1991b] C. A. J. Fletcher, Computational techniques for fluid dynamics, II: Specific techniques for different flow categories, Second ed., Springer, Berlin, 1991. MR 92f:76065

[Gilbarg and Hörmander 1980] D. Gilbarg and L. Hörmander, "Intermediate Schauder estimates", Arch. Rational Mech. Anal. 74:4 (1980), 297-318. MR 82a:35038 Zbl 0454.35022

[Gilbarg and Trudinger 2001] D. Gilbarg and N. S. Trudinger, Elliptic partial differential equations of second order, Springer, Berlin, 2001. MR 2001k:35004 Zbl 1042.35002

[John 1990] F. John, Nonlinear wave equations, formation of singularities, University Lecture Series 2, American Mathematical Society, Providence, RI, 1990. MR 91g:35001 Zbl 0716.35043

[Ladyzhenskaya and Ural'tseva 1968] O. A. Ladyzhenskaya and N. N. Ural'tseva, Linear and quasilinear elliptic equations, Academic Press, New York, 1968. MR 39 \#5941

[Lieberman 1987] G. M. Lieberman, "Local estimates for subsolutions and supersolutions of oblique derivative problems for general second order elliptic equations", Trans. Amer. Math. Soc. 304:1 (1987), 343-353. MR 88j:35061 Zbl 0635.35037

[Lieberman 1988] G. M. Lieberman, "Oblique derivative problems in Lipschitz domains, II: Discontinuous boundary data", J. Reine Angew. Math. 389 (1988), 1-21. MR 89h:35094 Zbl 0648.35033

[Lieberman and Trudinger 1986] G. M. Lieberman and N. S. Trudinger, "Nonlinear oblique boundary value problems for nonlinear elliptic equations", Trans. Amer. Math. Soc. 295:2 (1986), 509546. MR 87h:35114 Zbl 0619.35047

[Majda 1991] A. J. Majda, "One perspective on open problems in multi-dimensional conservation laws", pp. 217-238 in Multidimensional hyperbolic problems and computations (Minneapolis, MN, 1989), edited by J. Glimm and A. J. Majda, IMA Vol. Math. Appl. 29, Springer, New York, 1991. MR 91m:35142 Zbl 0769.35038

[Majda and Thomann 1987] A. Majda and E. Thomann, "Multidimensional shock fronts for second order wave equations", Comm. Partial Differential Equations 12:7 (1987), 777-828. MR 88k:35130 Zbl 0632.35047

[Maz'ja 1985] V. G. Maz'ja, Sobolev spaces, Springer, Berlin, 1985. MR 87g:46056 Zbl 0692. 46023

[Meĭrmanov 1980] A. M. Meŭrmanov, "The classical solution of a multidimensional Stefan problem for quasilinear parabolic equations", Mat. Sb. (N.S.) 112(154):2(6) (1980), 170-192. In Russian. MR 81j:35055

[Morawetz 1956] C. S. Morawetz, "On the nonexistence of continuous transonic flows past profiles, I”, Comm. Pure Appl. Math. 9 (1956), 45-68. MR 17,1149d

[Morawetz 1957] C. S. Morawetz, "On the nonexistence of continuous transonic flows past profiles, II”, Comm. Pure Appl. Math. 10 (1957), 107-131. MR 19,490e Zbl 0077.18901

[Morawetz 1958] C. S. Morawetz, "On the nonexistence of continuous transonic flows past profiles, III.”, Comm. Pure Appl. Math. 11 (1958), 129-144. MR 20 \#2961

[Morawetz 1964] C. S. Morawetz, "Nonexistence of transonic flow past a profile", Comm. Pure Appl. Math. 17 (1964), 357-367. MR 32 \#1994 Zbl 0125.43101

[Morawetz 1986] C. S. Morawetz, "Mathematical problems in transonic flow", Canad. Math. Bull. 29:2 (1986), 129-139. MR 87j:76065 Zbl 0572.76055

[Nazarov and Plamenevsky 1994] S. A. Nazarov and B. A. Plamenevsky, Elliptic problems in domains with piecewise smooth boundaries, de Gruyter Expositions in Mathematics 13, Walter de Gruyter, Berlin, 1994. MR 95h:35001 Zbl 0806.35001 
[Xin and Yin 2005a] Z. Xin and H. Yin, “Transonic shock in a nozzle, I: Two-dimensional case”, Comm. Pure Appl. Math. 58:8 (2005), 999-1050. MR 2006c:76079 Zbl 1076.76043

[Xin and Yin 2005b] Z. Xin and H. Yin, "Transonic shock in a nozzle, III: 2-D and 3-D complete Euler systems", Preprint, Institute of Mathematical Sciences at The Chinese University of Hong Kong, 2005.

[Xin et al. 2008] Z. Xin, Y. Wei, and H. Yin, "Transonic shock problem for the Euler system in a nozzle", Arch. Rat. Mech. Anal. (2008). To appear.

Received June 4, 2007. Revised January 23, 2008.

ZHOUPING XIN

DEPARTMENT OF MATHEMATICS

Chinese University OF HONG Kong

SHATIN

HONG KONG

zpxin@ims.cuhk.edu.hk

HUICHENG YIN

DEPARTMENT OF MATHEMATICS

NANJING UNIVERSITY

NANJING, 210093

CHINA

huicheng@nju.edu.cn 